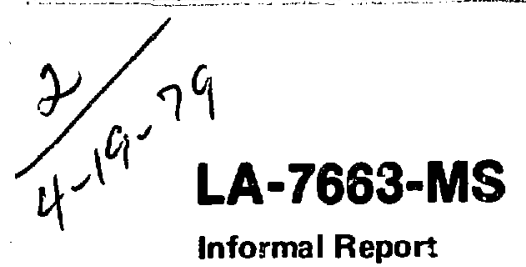

Informal Report

MASTER

Summary Documentation of LASL

Nuclear Data Evaluations for ENDF/B-V 
LA-7663-MS

Informal Report

Special Distribution

Issued: January 1979

\title{
Summary Documentation of L-ASL \\ Nuclear Data Evaluations for ENDF/B-V
}

\author{
Compiled by \\ P. G. Young
}

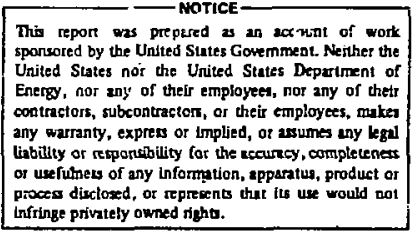

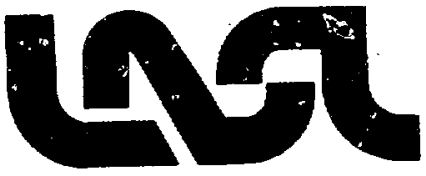


SUMMARY DOCUMENTATION OF LASL

NUCLEAR DATA EVALUATIONS FOR ENDF/B-V

Compiled by

P. G. Young

\begin{abstract}
Summaries are presented of nuclear data evaluations performed at Los Alamos Scientific Laboratory (LASL) that will comprise part of Version $V$ of the Evaluated Nuclear Data File, ENDF/B. A total of 18 general purpose evaluations of neutroninduced data are summarized, together with 6 summaries directed specifically at covariance data evaluations. The general purpose evaluation summaries cover the following isotopes: ${ }_{1-3} \mathrm{H}$, ${ }^{3},{ }^{4} \mathrm{He},{ }^{6}, 7 \mathrm{Li},{ }^{10} \mathrm{~B},{ }^{14}, 15 \mathrm{~N},{ }^{16} \mathrm{O},{ }^{27} \mathrm{Al},{ }^{182,183,184,186} \mathrm{~W},{ }^{23}{ }^{3} \mathrm{U}$, and ${ }^{242} \mathrm{Pu}$. The covariance data summaries are given for ${ }^{1} \mathrm{H},{ }^{6} \mathrm{Li}$, ${ }^{10} \mathrm{~B},{ }^{14} \mathrm{~N},{ }^{16} \mathrm{O}$, and ${ }^{27} \mathrm{Al}$.
\end{abstract}

\title{
I. INTRODUCTION
}

The utilization of new versions of the Evaluated Nuclear Data File, ENDF/B, which is issued through Brookhaven National Laboratory (BNL), is greatly enhanced by the availability on a timely basis of appropriate supporting documentation. Because comprehensive reports describing new evaluations are sometimes delayed in time, it has been the practice of BNL to collect shorter, summary documents from contributing evaluators and to provide this information for each new version of ENDF/B. The purpose of the present report is to provide the summary documents for evaluations performed at Los Alamos Scientific Laboratory (LASL) that will be a part of Version $V$ of ENDF/B. This report only summarizes evaluations of general purpose or covariance files to which LASL evaluators made recent and/or significant contributions. The summaries do not cover LASL work on such special purpose files as fission-product yields, activation cross sections, gas production, etc.

II. CONTENT OF SUMMARIES

A. General Purpose Files

Summary documentation for the general purpose file evaluations is given in Appendix A. These evaluations generally cover all neutron and gamma-ray production cross sections, angular distributions, and energy distributions for 
incident neutron energies in the range $10^{-5} \mathrm{eV}$ to $20 \mathrm{MeV}$ and may or may not $1 \mathrm{n}-$ clude covariance data. Summaries are given for the following isotopes: ${ }^{1-3} \mathrm{H}$,

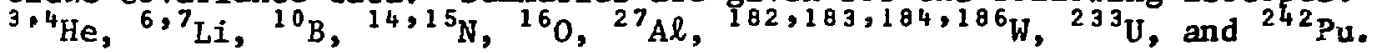

Each of the summary documents includes a section summarizing the extent of modification of the evaluation for version $V$, followed by a more detalled section that describes the major entries in each ENDF/B file for the various reaction types (MT numbers). In all cases the latter section is based upon the descriptive data in File 1 of the evaluation. For those isotopes that include neutron cross section standards, an additional section is given that summarizes the standards data in more detail. Graphical comparisons of evaluated and experimental data are included in several of the summaries.

B. Covariance Files

Additional documentation is provided in Appendix $B$ for the covariance data (ENDF/B File 33) evaluations of ${ }^{1} \mathrm{H},{ }^{6} \mathrm{Li},{ }^{10} \mathrm{~B},{ }^{14} \mathrm{~N},{ }^{16} \mathrm{O}$, and ${ }^{27} \mathrm{Al}$. The covariance summaries provide details as to the content and limitations of the correlated error data, together with brief descriptions of the methodology used for each of the error evaluations.

\section{ACKNOWLEDGMENTS}

It is a pleasure to thank H. M. Holleman for her help in interpreting the ENDF/B narrative descriptions and in typing the summaries.

I also wish to acknowledge the various evaluators for their efforts in providing detailed narrative files, which made the present task straightforward. 
APPENDIX A

Summary Documentation of LASL Nuclear

Data Evaluations for the General Purpose

Files of ENDF/B-V 


\title{
SUMMARY DOCUMENTATION FOR ${ }_{\text {H }}$
}

\author{
by \\ L. Stewart, R. J. LaBauve, and P. G. Young \\ Los Alamos Scientific Laboratory \\ Los Alamos, New Mexicc
}

\section{SUMMARY}

The ' $\mathrm{H}$ evaluation for ENDF/B-V (MAT 1301) is basically the same as the Version IV evaluation. Changes include the addition of correlated error data in $M F=33$ and different interpolation rules for $M T=1$ and 2 in $M F=3$. The evaluation covers the energy range $10^{-5} \mathrm{eV}$ to $20 \mathrm{MEV}$, and documentation is provided in LA-4574 (1971) and LA-6518-MS (197E).

\section{STANDARDS DATA}

The ${ }^{1} \mathrm{H}(\mathrm{n}, \mathrm{n}){ }^{1} \mathrm{H}$ elastic scattering cross section and angular distribution $(M F=3,4 ; M T=2)$ are standards in the energy region $1 \mathrm{keV}-20 \mathrm{MeV}$.

The extensive theoretical analysis of fast-neutron measurements by Hopkins and Breit ${ }^{1}$ was used to generate the scattering cross section and angular distributions of the neutrons for the ENDF/B-V file. ${ }^{2}$ The code and the Yale phase shifts ${ }^{3}$ were obtained from Hopkins ${ }^{4}$ in order to obtain the data on a fine-energy grid. Pointwise angular distributions were produced to improve the precision over that obtained from the published Legendre coefficients.* The phase shifts were also used to extend the energy range down below $200 \mathrm{keV}$ as represented in the original paper. 1

At: $100 \mathrm{eV}$, the elastic cross section calculated from the phase shifts is 20.449 barns, in excellent agreement with the thermal value of 20.442 derived by Davis and Barschall.5 Therefore, for the present evaluation, the free-atom scattering cross section is assumed to be constant below $100 \mathrm{eV}$ and equal to the value calculated from the Yale phase shifts at $100 \mathrm{eV}$ giving a thermal cross section of $20.449 \mathrm{~b}$.

Total cross-section measurements are compared with the evaluation in Fig. 1 for the energy range from $10 \mathrm{eV}$ to $0.5 \mathrm{MeV}$. Similarly, Figs. 2 and 3 compare the evaluation with measured data from 0.5 to $20 \mathrm{MeV}$. The agreement with the earlier experiments shown in $\mathrm{Fig} .2$ is quite good over the entire energy range. The 1969 data of Schwartz ${ }^{6}$ included in Fig. 3, however, lie slightly below the evaluation over most of the energy range even though agreement with the 1972 results of clement ${ }^{7}$ is quite acceptable.

\footnotetext{
*For $E_{n}=30 \mathrm{MeV}$, the difference in the $180^{\circ}$ cross section is $\sim 1 \%$ as calculated from the Legendre coefficients ${ }^{3}$ compared to that calculated from the phase shifts.
} 
Unfortunately, few absolute values of the angular dependence of the neutrons (or recoil protons) exist and even the relative measurements are often restricted to less than half of the angular range. The experiment of $0^{\circ}{ }^{8}$ at $3.1 \mathrm{MeV}$ is not atypical of the earlier distributions which, as shown in Fig. 4, does not agree with the phase-shift predictions. Near $14 \mathrm{MeV}$, the $T(d, n)$ neutron source has been employed in many experiments to determine the angular distributions. A composite of these measuraments is compared with ENDF/B-V in Fig. 5A. Note that most of the experiments are in reasonable agreement on a relative scale, but $10 \%$ discrepancies frequently appear among the data sets. The measurements of Cambou $^{9}$ average more than $5 \%$ lower than the predicted curve and differences of $5 \%$ or more are occasionally apparent among the data of a single set. Figure 5B shows the measurements of Calonsky ${ }^{10}$ at $17.9 \mathrm{MeV}$ compared with the evaluation. Again, the agreement on an absolute basis is quite poor.

Elastic scattering angular distributions at $0.1,5,10,20$, and $30 \mathrm{MeV}$ are provided in Ref. 11 as Legendre expansion coefficients. Using the Hopkins-Breit phase-shift program and the Yale phase shifts, additional and intermediate energy points were calculated for the present evaluation. ${ }^{2}$ As shown in Figs. 5-16 of Ref. 2, the angular distributions are neither isotropic below $10 \mathrm{MeV}$ nor symmetric about $90^{\circ}$ above $10 \mathrm{MeV}$ as assumed in earlier evaluations. In this evaluation, the angular distribution at $100 \mathrm{keV}$ is assumed to be isotropic since the calculated $180^{\circ} / 0^{\circ}$ ratio is very neariy unity, that is, 1.0011 . At $500 \mathrm{keV}$, this ratio approaches 1.005 . Therefore, the pointwise normalized probabilities as a function of the center-of-mass scattering angle are provided at the following energies: $10^{-5} \mathrm{eV}$ (isotropic), $100 \mathrm{keV}$ (isotropic), $500 \mathrm{keV}$, and at 1-MeV intervals from 1 to $20 \mathrm{MeV}$.

Certainly the Hopkins-Breit phase shifts reproduce reasonabiy well the measured angular distributions near $14 \mathrm{MeV}$. It is important, however, that experiments be made at two or three energies which would, hopefully, further corroborate this analysis. Near $14 \mathrm{MeV}$, the energy-dependent total cross section is presently assumed to be known to $\sim 1 \%$ and the angular distribution to $\sim 2-3 \%$. At lower energies where the angular distributions approach isotropy, the error estimate on the angular distribution is less than $1 \%$.

It should be pointed out that errors involved in using hydrogen as a standard lepend upon the experimental techniques employed and therefore may be significantly larger than the errors placed on the standard cross section. The elastic angular distribution measurements of neutrons scattered by hydrogen, which are available today, seem to indicate that $\sigma(\theta)$ is difficult to measure with the precision ascribed to the reference standard. If this is the case, then the magnitude of the errors in the $\sigma(\theta)$ measurements might be indicative of error assignments which should be macie on hydrogen flux monitors. That is, it is difficult to assume that hydrogen scattering can be implemented as a standard with much higher precision than i.t can be measured. Even though better agreement with many past measurements can be reached by renormalizing the absolute scales, such action may not always be watranted.

At this time, no attempt has been made to estimate the effect of errors on the energy scale in ENDF/B. It is clear, however, that a small energy shift would produce a large change in the cross section, especially at low energies. For example, a 50-keV shift in energy near $1 \mathrm{MeV}$ would produce a change in the standard cross section of approximately $2 \frac{1}{2} \%$. Therefore, precise determination of the incident neutron energy and the energy spread could be very important in employing hydrogen as a cross-section standard, depending upon the experimental technique. 
III. ENDF/B-V FILES

File 1. General Information

MT=451. Descriptive data.

File 2. Resonance Parameters

$M T=151$. Effective scattering radius $=1.27565 \times 10^{-12} \mathrm{~cm}$.

Resonance parameters not given.

Eile 3. Neutron Cross Sections

$\mathrm{MT}=1$. Total Cross Sections

The total cross sections are obtained by adding the elastic scattering and radiative capture cross sections at a11 energies, 1.OE-05 eV to $20 \mathrm{MeV}$.

MT=2. Elastic Scattering

Standard - see discussion in Sec. II.

MT=102. Radiative Capture

These cross sections are taken from the publication of A. Horsley where a value of $332 \mathrm{mb}$ was adopted for the thermal value. See Ref. 51.

$\mathrm{MT}=251$. Average Value of Cosine of Scattering Angle In Lab System from 1.0E-05 Ev to $20 \mathrm{MeV}$. (Provided by BNL).

$\mathrm{MT}=252$. Average Logarithmic Energy Change Per Collision, from 1.0E-05 eV to $20 \mathrm{MeV}$. (Provided by BNL).

$\mathrm{MT}=253$. Gamma, from $1.0 \mathrm{E}-05 \mathrm{eV}$ to $20 \mathrm{MeV}$. (Provided by BNL).

File 4. Neutron Angular Distributions

$\mathrm{MT}=2$. Neutron elastic scattering angular distributions in the center of mass system, given as normalized pointwise probabilities. See Sec. II above.

File 7. Thermal Neutron Scattering Law Data

$\mathrm{MT}=4$. 0.00001 to $5 \mathrm{eV}$ free gas sigma $=20.449$ barns.

File 12. Ganma Ray Multiplicities

$\mathrm{MT}=102$. Radiative Capture Multiplicities.

Multiplicity is unity at all neutron energies. $L P=2$ is now implemented; therefore, all gamma energies must be calculated. 
File 14. Gamma Ray Angular Distributions

MT=102. Radiative capture angular distribution

Assumed isotropic at all neutron energies.

\section{File 33. Correlated Errors}

$\mathrm{MT}=1$. Covariance matrix derived from $M T=2,102$.

$\mathrm{MT}=2$. Covariance data added for the elastic scattering by D. G. Foster, Ji. (Jan. 77).

$M T=102$. Covariance data for radiative capture added by P. G. Young (Nov. 7, 1978).

\section{REFERENCES}

1. J. C. Hopkins and G. Breit, "The $H(n, n) H$ Scattering Observables Required for High Precision Fast-Neutron Measurements," Nuclear Data A $\underline{9}, 137$ (1971) and private communication prior to publication (1970).

2. L. Stewart, R. J. LaBauve, and P. G. Young, "Evaluated Nuclear Data for Hydrogen in the ENDF/B-II Format," LA-4574 (1971). Neither the cross sections nor the angular distributions have been changed since ersion II except to add one more significant figure to the total cross section.

3. R. E. Seamon, K. A. Friedman, G. Breit, R. D. Haracz, J. M. Holt, and A. Prakash, Phys. Rev. 165, 1579 (1968).

4. J. C. Hopkins, private communication to L. Stewart (1970).

5. J. C. Davis and H. H. Barschall, "Adjustment in the n-D Singlet Effective Range," Phys. Lett. 27B, 636 (1968).

6. R. B. Schwartz, R. A. Schrack, and H. T. Heaton, "A Search for Structure in the n-p Scattering Cross Section," Phys. Lett. $\underline{30}, 36$ (1969).

7. J. M. Clement, P. Stoler, C. A. Goulding, and R. W. Fairchild, "Hydrogen and Deuterium Total Neutron Cross Sections in the MeV Region," Nucl. Phys. a 183, 51 (1972).

8. Y. Oda, J. Sanada, and S. Yamabe, "On the Angular Distribution of 3.1-MeV Neutrons Scattered by Protons," Phys. Rev. 80, 469 (1950).

9. F. Cambou, "Anelioratin des Methods de Spectrumetrie des Neutrons Rapids," Thesis - U. of Paris, CEA-N-2002 (1961).

10. A. Galonsky and J. P. Judish, "Angular Distribution of n-p Scattering at $17.9 \mathrm{MeV}, "$ Phys. Rev. 100, 121 (1955).

11. "ENDF/B Summary Documentation," ENDF-201, Compiled by D. Garber, (October 1975). 
12. E. Melkonian, "Slow Neutron Velocity Spectrometer Studies of $\mathrm{O}_{2}, \mathrm{~N}_{2}, \mathrm{~A}, \mathrm{H}_{2}$, $\mathrm{H}_{2} \mathrm{O}$, and Seven Hydrocarbons," Phys. Rev. 76, 1950 (1949).

13. D. H. Frisch, "The Total Cross Sections of Carbon and Hydrogen for Neutrons of Energies from 35 to $490 \mathrm{keV}$," Phys. Rev. 70, 589 (1946).

14. W. D. Allen and A. T. G. Ferguson, "The n-p Cross Section in the Range 60$550 \mathrm{keV}, "$ Proc. Phys. Soc. (London) 68, 1077 (1955).

15. E. Bretscher and E. B. Martin, "Determination of the Collision Cross-Section of $\mathrm{H}$, Deuterium, $\mathrm{C}$ and $\mathrm{O}$ for Fast Neutrons," Helv. Phys. Acta 23, 15 (1950).

16. C. E. Engleke, R. E. Benenson, E. Melkonian, and J. M. Lebowitz, "Precision Measurements of the n-p Total Cross Section at 0.4926 and $3.205 \mathrm{MeV}^{\text {, }}$ "Phys. Rev. 129, 324 (1963).

17. C. L. Bailey, W. E. Bennett, T. Bergstralh, R. G. Nuckolls, H. T. Richards, and J. H. Williams, "The Neutron-Proton and Neutron-Carbori Scattering Cross Sections for Fast Neutrons," Phys. Rev. 70, 583 (1946).

18. E. E. Lampi, G. Frier, and J. H. Williams, "Total Cross Section of Carbon and Hydrogen for Fast Neutrons," Phys. Rev. 76, 188 (1949).

19. W. E. Good and G. Scharff-Goldhaber, "Total Cross Sections for 900-keV Neutrons," Phys. Rev. 59, 917 (1941).

20. S. Bashkin, B. Petree, F. P. Mooring, and R. E. Peterson, "Dependence of Neutron Cross Sections on Mass Number," Phys. Rev. 77, 748 (1950).

21. R. E. Fields, R. L. Becker, and R. K. Adair, "Measurement of the NeutronProton Cross Section at 1.0 and $2.5 \mathrm{MeV}$," Phys. Rev. 94, 389 (1954).

22. C. L. Storrs and D. H. Frisch, "Scattering of $1.32 \mathrm{MeV}$ Neutrons by Protors," Phys. Rev. 95, 1252 (1954).

23. D. G. Foster, Jr. and D. W. Glasgow, "Neutron Totäl Cross Sections, 2.5-15 MeV, Part 1 (Experimental)," Nucl. Instr. and Methods, 36, 1 (1967).

24. R. E. Fields, "The Total Neutron-Proton Scattering Cross Section at 2.5 MeV," Phys. Rev. 89, 908 (1953).

25. G. Ambrosina and A. Sorriaux, "Total Cross Section Efficiency for Carbon, Gluorine and Vanadium," Comptes Rendus 260, 3045 (1965).

26. W. H. Zinn, S. Seely, and V. W. Cohen, "Collision Cross SEctions for D-D Neutrons," Phys. Rev. 56, 260 (1939).

27. N. Nereson and S. Darden, "Average Neutron Total Cross Sections in the 3to 12- MeV Region," Phys. Rev. 94, 1678 (1954).

28. E. M. Hafner, W. F. Hornyak, C. E. Falk, G. Snow, and T. Coor, "The Total n-p Scattering Cross Section at $4.75 \mathrm{MeV}, "$ Phys. Rev. 89, 204 (1953). 
29. W. Sleator, Jr., "Collision Cross Sections of Carbon and Hydrogen for Fast Neutrons," Phys. Rev. 72,207 (1947).

30. A. Bratenah1, J. M. Peterson, and J. P. Stoering, "Neutron Total Cross Sections in the 7- to 14-MeV Region," UCRL-4980 (1957).

3.1. A. H. Lasday, "Total Neutron Cross Sections of Several Nuclei at 14 MeV," Phys. Rev. $\underline{8}^{\urcorner}, 139$ (1951).

32. L. S. Goodman, "Total Cross Sections for 14-MeV Neutrons," Phys. Rev. 88, 686 (1952).

33. H. L. Poss, E. O. Salant, and L. C. L. Yuan, "Total Cross Sections of Carbon and Hydrogen for 14-MeV Neutrons," Phys. Rev. 85, 703 (1951).

34. M. Tanaka, N. Koori, and S. Shirato, "Differential Cross Sections for Neutron-Proton Scattering at $14.1 \mathrm{MeV}, " \mathrm{~J}$. Phys. Soc. (Japan) 28, 11 (1970).

35. M. E. Battat, R. O. Bondeiid, J. H. Coon, L. Cranberg, R. B. Day, F. Edeskuty, A. H. Frentrop, R. L. Henkel, R. L. Mills, R. A. Nobles, J. E. Perry, D. D. Phillips, T. R. Roberts, and S. G. Sydoriak," Total Neutron Cross Sections of the Hydrogen and Helium Isotopes," Nucl. Phys. 12, 291 (1959).

36. J. C. Allred, A. H. Armstrong, and L. Rosen, "The Interaction of 14-MeV Neutrons with Protons and Deuterons," Phys. Rev. 91, 90 (1953).

37. C. F. Cook ard T. W. Bonner, "Scattering of Fast Neutrons in Light Nuclei," Phys. Rev. 94, 651 (1954).

38. H. L. Poss, E. O. Salant, G. A. Snow, and L. C. L. Yuan, "Total Cross Sections for 14-MeV Neutrons," Phys. Rev. 87, 11 (1952).

39. P. H. Bowen, J. P. Scanlon, G. H. Stafford, and J. J. Thresher, "Neutron Total Cross Sections in the Energy Range 15 to $120 \mathrm{MeV}$," Nucl. Phys. 22, 640 (1961).

40. J. M. Peterson, A. Bratenah1, and J. P. Stoering, "Neutron Total Cross Sections in the 17- to 29-MeV Range," Phys. Rev. 120, 521 (1960).

41. D. E. Groce and B. D. Sowerby, "Neutron-Proton Total Cross Sections Near 20, 24, and $28 \mathrm{MeV}$," Nuc1. Phys. 83, 199 (1966).

42. M. L. West II, C. M. Jones, and H. B. Willard, "Total Neutron Cross Sections of Hydrogen and Carbon in the 20-30 MeV Region," ORNL-3778, 94 (1.965).

43. R. B. Day, R. I. Mills, J. E. Perry, Jr., and F. Schreb, "Total Cross Section for n-p Scattering at $20 \mathrm{MeV}, "$ Phys. Rev. 114, 209 (1959).

44. R. B. Day and R. L. Henkel, "Neutron Total Cross Sections at $20 \mathrm{MeV}$," Phys. Rev. 92, 358 (1953). 
45. M. E. Remley, W. K. Jentschke, and P. G. Kruger, "Neutron-Proton Scattering Using Organic Crystal Scintillation Detectors," Phys. Rev. 89, 1194 (1953).

$46 \mathrm{~J}$ 。 D. Seagrave, "Recoil Deuterons and Disintegration Protons from the $\mathrm{n}-\mathrm{d}$ Interaction, and $n-p$ Scattering at $E_{\mathfrak{n}}=14.1 \mathrm{MeV}, "$ Phys. Rev. 97, 757 (1955).

47. S. Shirato and K. Saitch, "On The Differential Cross Section for NeutronProton Scattering at $14.1 \mathrm{MeV}, "$ J. Phys. Soc. (Japan) 36, 331 (1974).

48. T. Nakamura, "Angular Distribution of n-p Scattering at $14.1 \mathrm{MeV}, " \mathrm{~J}$. Phys. Soc. (Japan) 15, 1359 (1960).

49. A. Suhami and R. Fox, "Neutron-Proton Small Angle Scattering at $14.1 \mathrm{MeV}, "$ Phys. Lett. 24, 173 (1967).

50. I. Basar, "Elastic Scattering of $14.4 \mathrm{MeV}$ Neutrons on Hydrogen Isotopes," Few Body Problems Light Nuclei/Nucl. Interactions, Brela, 867 (1967).

51. A. Horsley, "Neutron Cross Sections of Hydrogen in the Energy Range 0.0001 eV-20 MeV," Nucl. Data A2, 243 (1966).

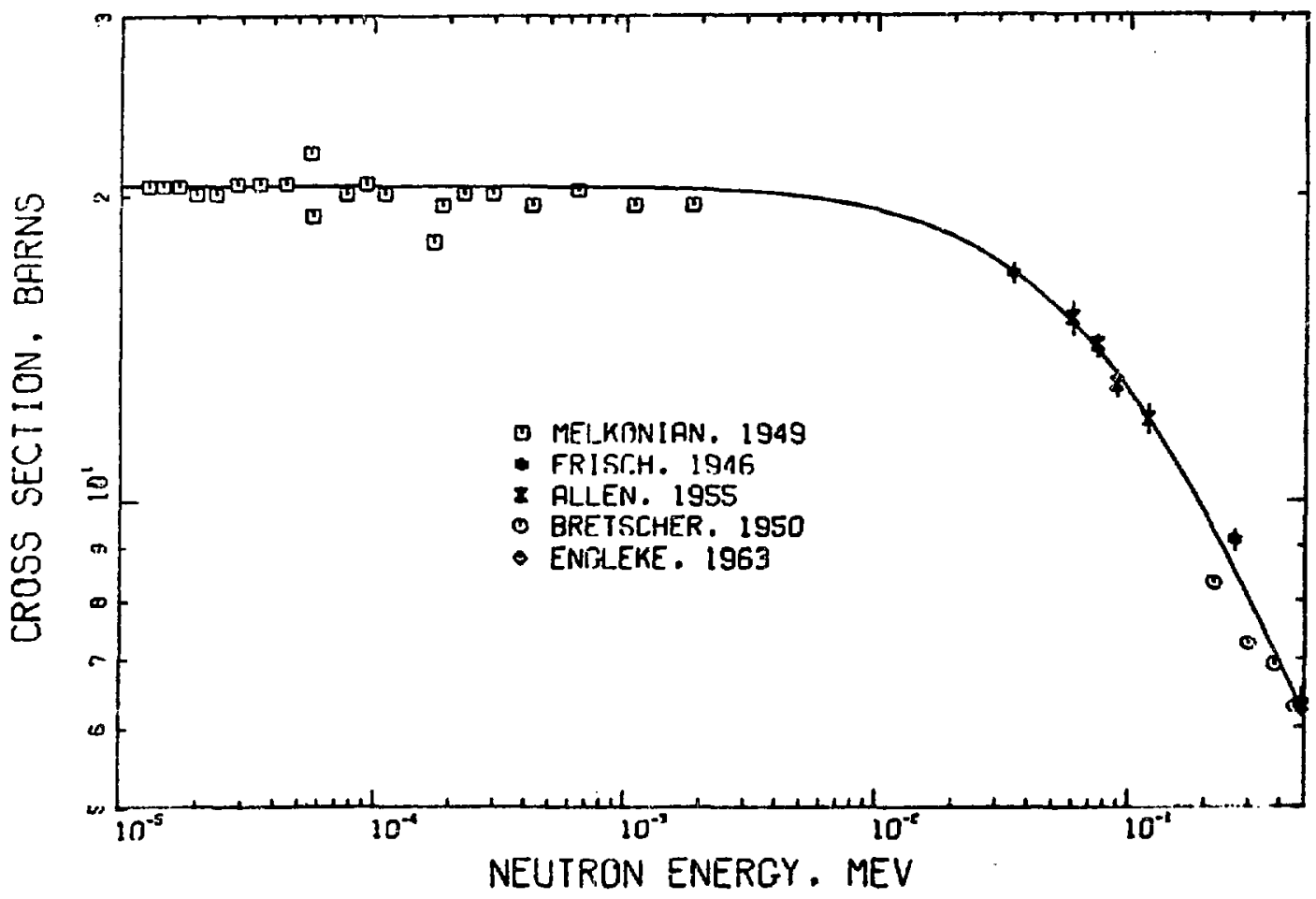

Fig. 1.

Total cross section for hydrogen from $1 \times 10^{-5} \mathrm{eV}$ to $500 \mathrm{keV}$. The ENDF/B-V evaluation is compared to the measurements of Refs, 12-16. 

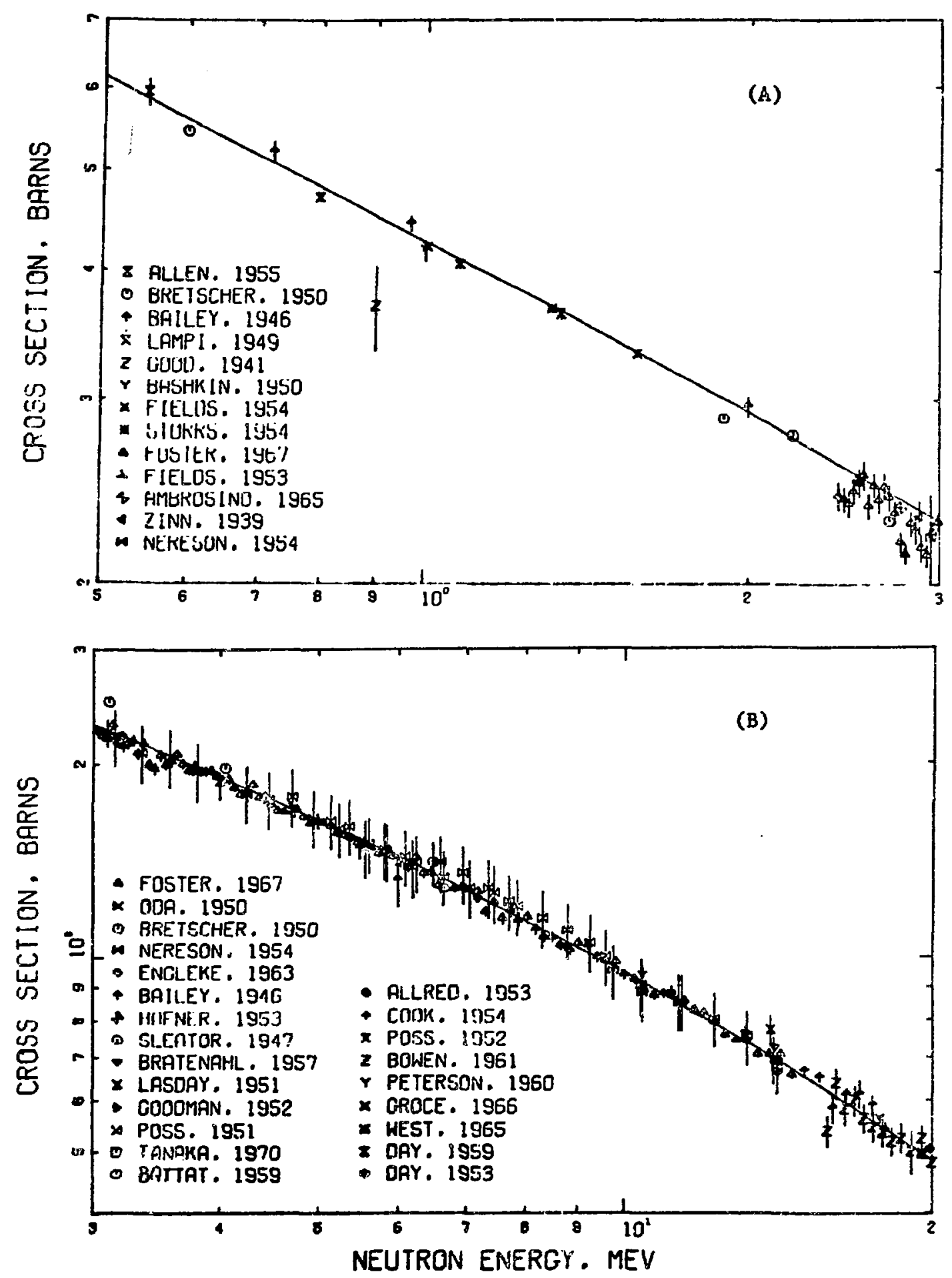

Fig. 2.

Total cross section for hydrogen from $500 \mathrm{keV}$ to $20 \mathrm{MeV}$. The ENDF/B-V evaluation is compared to measurements reported in Refs, 8, 14-44. 

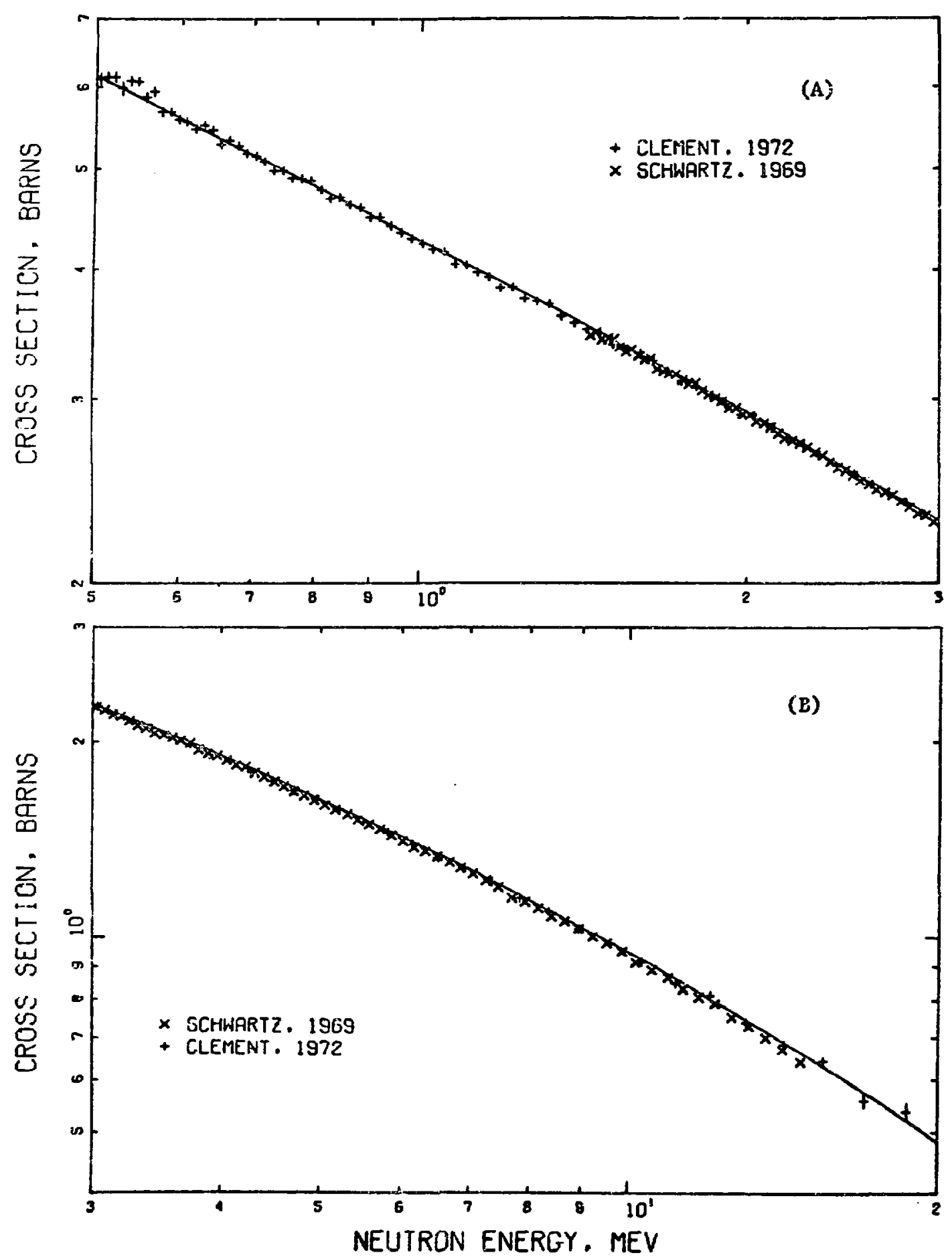

Fig. 3.

Total cross section for hydrogen from $500 \mathrm{keV}$ to $20 \mathrm{MeV}$. The ENDF/B-V evaluation is compared to measurements reported in Refs. 6 and 7. 


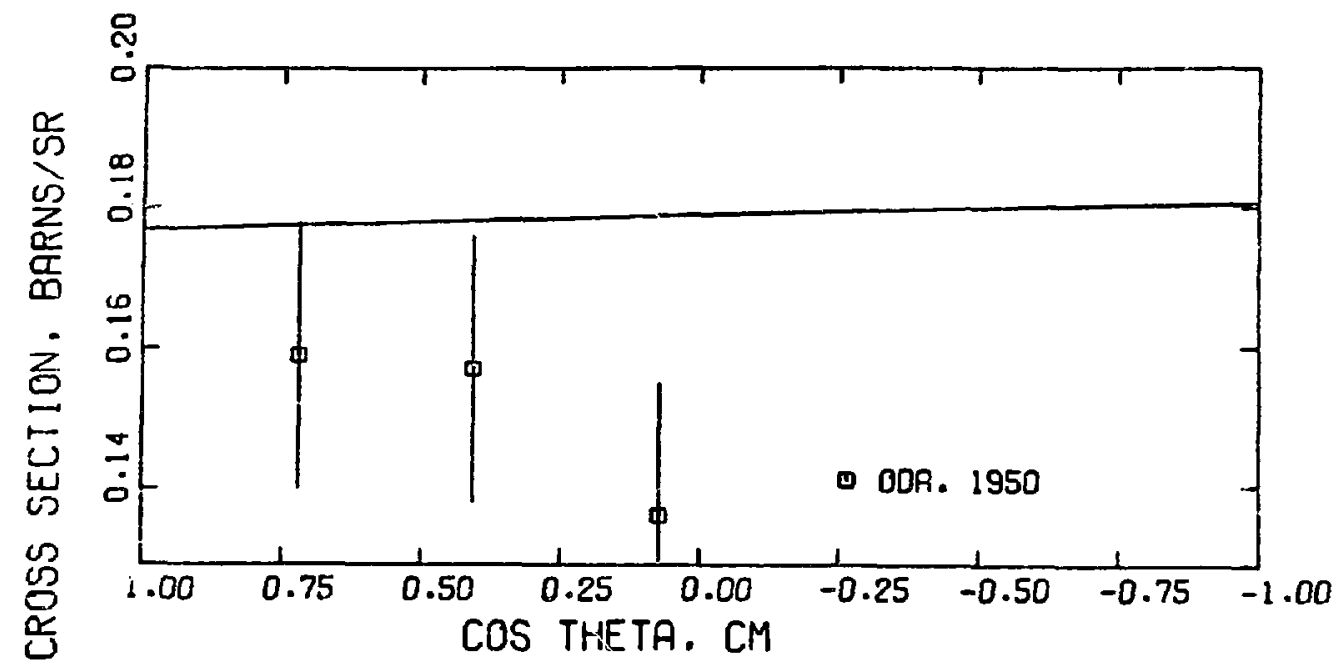

Fig. 4.

Angular distribution of the neutrons elastically scattered from hydrogen at $3.1 \mathrm{MeV}$. ENDF/B-V is compared with the experimental values of oda. ${ }^{8}$

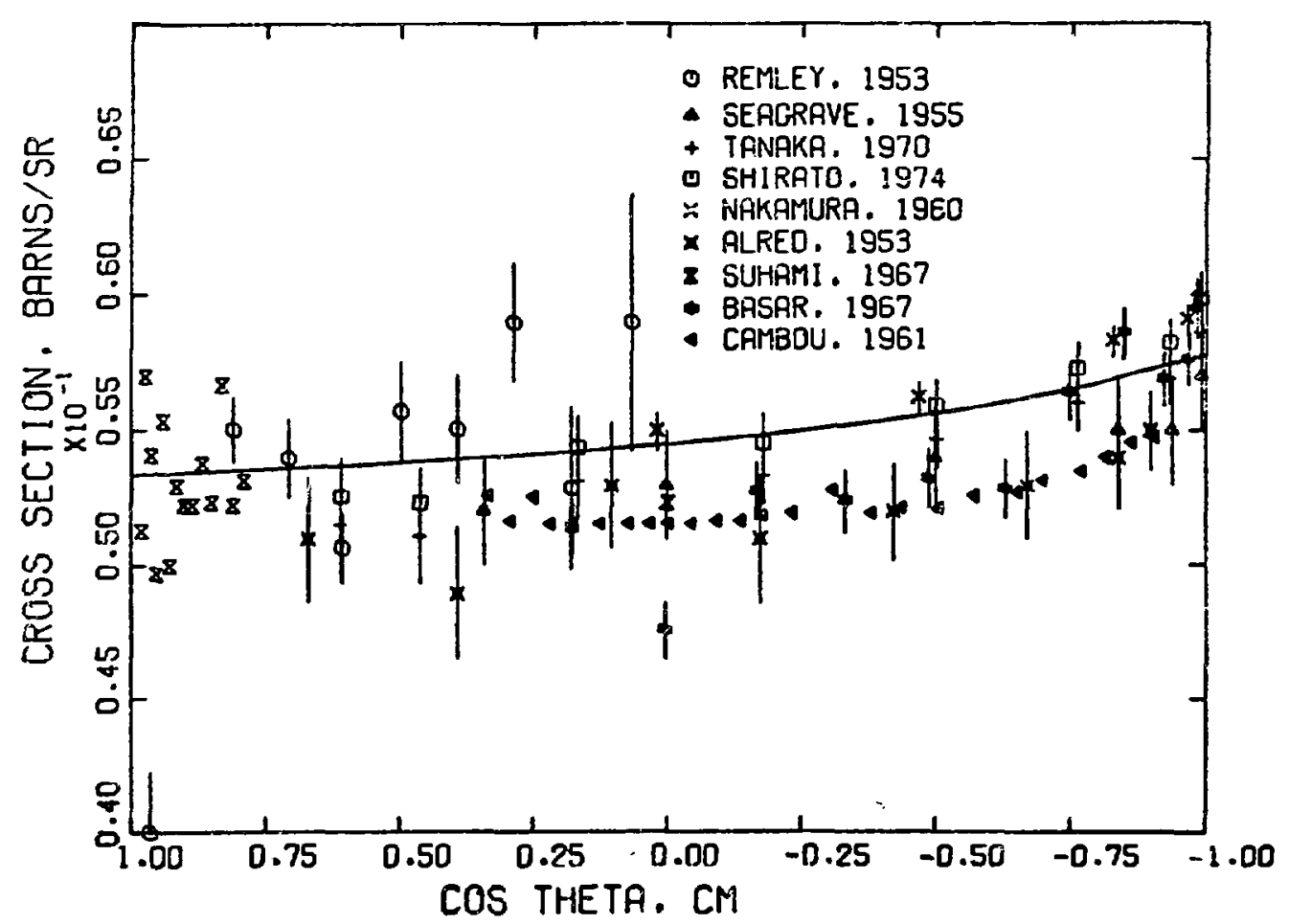

Fig. 5A.

Angular distribution of the neutrons elastically scattered from hydrogen at energles near $14 \mathrm{MeV}$. The experimental data shown were reported in Refs. 9, 34, 36 and 45-50. 


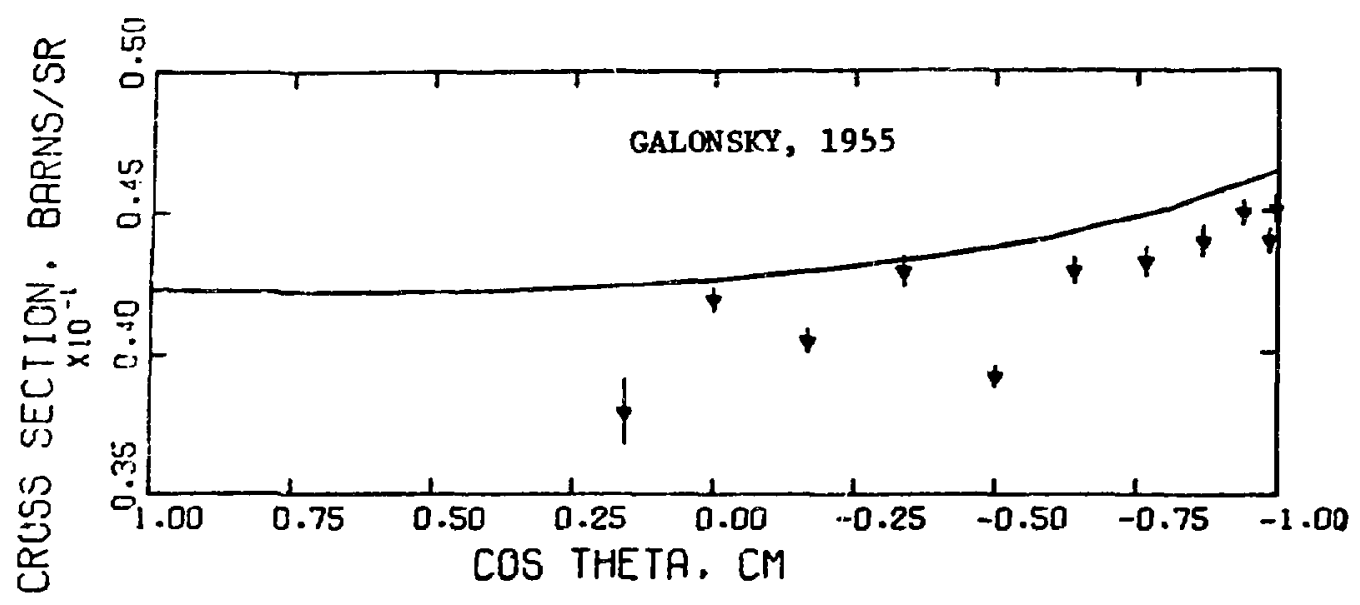

Fig. 5B.

Angular distribution of the neutrons elastically scattered from hydrogen at energies near $17.8 \mathrm{MeV}$. The experimental data shown were reported in Ref. 10. 
SUMMARY DOCUNENTATION FOR ${ }^{2}$ H

\author{
by \\ L. Stewart and A. Horsley ${ }^{+}$ \\ Los Alamos Scientific Laboratory \\ Los Alamos, New Mexico
}

\title{
I. SUMMARY
}

The ENDF/B-V evaluation (MAT=1302) j.s entirely different from the Version IV data set and is based upon a revision of an earlier evaluation given in LA-3271 (1968). The main change made to the earlier evaluation was to modify the total and elastic cross sections below $500 \mathrm{keV}$ in order to more closely reflect experimental data from RPI. In addition, Files 8 and 9 have been included to provide tritium production information. The evaluation covers the energy range $10^{-5} \mathrm{eV}$ to $20 \mathrm{MeV}$.

\section{ENDF/B-V FILES}

File 1. GENERAL INFORMATION

MT-451. Descriptive data.

File 2. Resonance Parameters

$\mathrm{MT}=151$. Effective scattering radius $=0.51977 \times 10^{-12} \mathrm{~cm}$. Resonance parameters not given.

\section{File 3. Neutron Cross Sections}

MT=1. Total Cross Section

All data plotted and compared up to 1967 in LA-3271. Changes incorporated below $1.5 \mathrm{MeV}$ but evaluation does not agree with lowenergy experiments at $\mathrm{NBS}^{2}$ (which are preliminary) but agrees at higher energies. The Davis data ${ }^{3}$ show a peculiar drop of a few per cent from 3.5 to $9 \mathrm{MeV}$ but agree above and below these energies.

MT=2. Elastic Cross Sections

Data obtained from integrating $\mathrm{n}-\mathrm{D}$ and $\mathrm{P}-\mathrm{D}$ angular distributions. Since the radiative capture is in microbarns, the elastic is

\footnotetext{
$\overline{干_{\text {Atomic Weapons }}}$ Research Establishment, Aldermaston, U.K.
} 
essentially equal to the total cross se:tion below the $n, 2 n$ threst.old and the total minus the $n, 2 n$ above the threshold. Checks and balances were always made. See LA-3271 for the graphical comparisons.

$\mathrm{MT}=16 . \quad(\mathrm{n}, 2 \mathrm{n})$ Cross Eection

Data taken from Holmberg ${ }^{4}$ and from Catron. ${ }^{5}$ See LA-3271. Nothing is known about the cross section above $14 \mathrm{MeV}$.

MT=102. Radiative Capture Cross Section

The thermal cross section is 506 microbarns which was extrapolated as $1 / \mathrm{V}$ up to $1 \mathrm{keV}$. Curve was drawn above this energy to include measurements on the inverse reaction by Bösch. ${ }^{6}$ The $14 \mathrm{MeV}$ value is a factor of 3 lower than Cerineo. ${ }^{7}$ See LA-3271 for graphical results.

File 4. Neutron Angular Distributions

$\mathrm{MT}=2$. Elastic Angular Distributions

Taken from $n-D$ and $P-D$ scattering. Agreement is good with van Oers analysis. ${ }^{8}$ See LA-3271.

MT=16. $(n, 2 n)$ Angular Distributions

Calculated by code of Young ${ }^{9}$ assuming phase space argument, therefore ignoring the observations of the virtual deuteron. See LA-327I for comparisons with $n-D$ and $p-D$ breakup spectra.

File 5. Neutron Energy Distributions

$\mathrm{MT}=16$. (n,2n) Energy Distribution

Discussed under $M=4$. Energy distributions calculated assuming pure phase space model.

File 8, 9. Decay Data

MT=102. Decay Information Added For Tritium Production

File 12. Gamma Ray Multiplicities

MT=102. $(n, \gamma)$ Multiplicity

Assumed a single gamma emitted at all energies. Employed the $L P=2$ flag to conserve energy.

File 14. Gamma Ray Angular Distributions

MT $=102$. ( $n, \gamma)$ Angular Distribution

Assumed isotropic. 


\section{REFERENCES}

1. P. Stoler, N. N. Kausha1, F. Green, E. Harms, and L. Laroze, Phys. Rev. Lett. 29, 1745 (1972).

2. R. B. Schuartz, National Bureau of Standards personal communicatinn ( 1977$)$.

3. J. C. Davis and H. H. Barscha11, Phyis, Rev, C3, 1798 (1971).

4. M. Holmberg and J. Hansen, IAEA Conference on Nuclear Data, "Microscopic Cross Sections and Other Data Basis for Nuclear Reactors," Paris, October $17-21$, 1966, paper $\mathrm{CN}-23 / 18$.

5. H. C. Catron, M. D. Goldberg, R. W. Hill, J. M. LeBlanc, J. F. Stoering, C. J. Taylor, and M. A. Williamson, Phys. Rev. 123, 218 (1961).

6. R. Bösch, J. Lang, R. Müller, and Wölfli, Phys. Lett. 8, 120 (1964).

7. M. Cerineo, K. Ilakovac, I. Slaus, and P. Tomas, Phys. Rev. I24, 1947 (1961).

8. W. T. H. van Ders and K. W. Broekman, Jr., Nuc1. Phys. 21, 189 (1960).

9. P. G. Young, Los Alamos Scientific Laboratory, personal communication (1977). 
SUMMARY DOCUMENTATION FOR ${ }^{3} \mathrm{H}$

\author{
by \\ L. Stewart \\ Los Alamos Scientific Laboratory \\ Los Alamos, New Mexico
}

\title{
I. SUMMARY
}

The ENDF/B-V evaluation for ${ }^{3} \mathrm{H}(\mathrm{MAT}=1169)$ is identical to the Version IV evaluation except for the transfer of the decay data from File 1 to File 8 . Gamma-ray production data are not included because the radiative capture and $\left(n, n^{\prime}\right)$ cross sections are assumed to be negligibly small at all energies. The evaluation covers the energy range $10^{-5} \mathrm{eV}$ to $20 \mathrm{MeV}$ and is documented in LA-3270 (1965).

\section{ENDF/B-V FILES}

File 1. General Informatio:2

MT-451. Descriptive data.

File 2. Resonance Parameters

$\mathrm{MT}=151$. Effective scattering radius $=0.32164 \times 10^{-12} \mathrm{~cm}$. Resorance parameters not given.

\section{File 3. Neutron Cross Sections}

MT=1. Total Cross Sections

Total cross sections from $290 \mathrm{keV}$ to $20 \mathrm{MeV}$ from LASL measurements (Ref. 1). Estimated below $290 \mathrm{keV}$.

MT=2. Elastic Scattering Cross Section

Data taken from measurements (Refs. 2 to 10) up to 1967 on $n-T$ and $\mathrm{p}-\mathrm{He}^{3}$ systems. Also, recent measurements on $\mathrm{n}-\mathrm{T}$ by Seagrave et al. (Ref. 11.) and on $\mathrm{p}-\mathrm{He}^{3}$ by Morales and Cahill (Ref. 12) and by Hutson et ail. (Ref. 13) have been used to update the elastic angular distributions above $15 \mathrm{MeV}$. (February 1971) (this is the only change made to the evaluation from that described in LA-3270). 


\section{MT=16. $(n, 2 n)$ Cross Section}

Only one measurement exists, that of Mather and Pain (Ref. 14) at 14.1 MeV. Estimates of this cross section were therefore made from systematics and the $\mathrm{p}-\mathrm{He}^{3}$ reaction studies of Rosen and Leland (Ref. 9) and of Anderson (Ref. 15).

$\operatorname{MT}=17$. (n,3n) Cross Section

No data given since estimates from isospin considerations give essentially zero probabilities. Mather and Pain (Ref. 14) and Cookson (Ref, 16) confirm these estimates.

$\mathrm{MT}=102$. (n, $\gamma$ ) Cross Section (not included)

The measured cross section is less than or equal to 6.7 microbarns at thermal (the sign of the Q-value is uncertain). This cross section is therefore assumed zero at all energ 5 .

$\mathrm{MT}=251$. Average Value of the Scattering Angle in the raboratory System from $1.0 \mathrm{E}-05 \mathrm{eV}$ to $20 \mathrm{MeV}$

$\mathrm{MT}=252$. Average Logarithmetic Energy Change Per Collision, From $1.0 \mathrm{E}-05 \mathrm{eV}$ to $20 \mathrm{MeV}$

$\mathrm{MT}=253$. Gamma, From $1.0 \mathrm{E}-05 \mathrm{eV}$ to $20 \mathrm{MeV}$

File 4. Neutron Angular Distributions

MT=2. Elastic Angular Distributions

These are given in the center-of-mass system as normalized probabilities versus cosine of the scattering angle.

$\mathrm{MT}=16$. $(\mathrm{n}, 2 \mathrm{n})$ Angular Distributions

These are given in the laboratory system as normalized probabilities versus cosine of the scattering angle. See LA-3270 for details on these distribution functions.

File 5. Neutron Energy Distributions

$M T=16$. $(n, 2 n)$ Energy Distributions

These are given as normalized probabilities versus energy of the out going neutron in the laboratory system. See LA-3270 for details on these distribution functions.

File 8. Decay Data

MT=457. Decay Dara Provided by C. Reich (INEL), Based On Chart of Nuclides, Wapstra's Mass Tables, and Nuclear Data Tables. Placed in ENDF File and Format by BNL and LASL. 
Files 12 - 15. Gamma Ray Data (not included)

Only radiative capture produces gamma rays. Since the capture cross section is assumed zero at all energies, these files are purposely left empty.

\section{REFERENCES}

1. Los Alamos Physics and Cryogenics Groups, Nucl. Phys. 12, 291 (1959).

2. J. D. Seagrave et al., Phys. Rev. 119, 1981 (1960).

3. J. H. Coon et al., Phys. Rev. 81, 33 (1951).

4. T. B. Clegg et al., Nucl. Phys. 50, 621 (1964).

5. W. Haeberli et al., Phys. Rev. 133, 81178 (1964).

6. J. E. Brolley, Jr., et al., Phys. Rev. 117, 1307 (1960). Data at back angles at which $\mathrm{He}^{3}$ particles were observed were ignored in this analysis.

7. R. H. Lovberg, Phys. Rev. 103, 1393 (1956).

8. R. A. Vanetsian and E. D. Fedchenko, Sov. J. of Atomic Energy, Trans. 2, 141 (1957), and K. P. Artemov, S. P. Calinin and L. H. Samoilov, JETP 10, 474 (1960). The latter data were not used, since they covered the 5- to $10-\mathrm{MeV}$ energy region and were not available.

9. L. Rosen and W. T. Leland, Wash-1064 (EANDC(US)-79U) (0ct. 1966), p. 99.

1C. D. Blanc, F. Cambou, M. Niel, and G. Vedrenne, J. De Physique, Supplement Fasc. 3-4, C1-98 (1966).

11. J. D. Seagrave et a1., LA-DC-12954 (1971).

12. J. R. Morales and T. A. Cahill, Bull. Am. Phys. Soc. 14, 554 (1969), and private communication.

13. R. L. Hutson et al., Phys. Rev. 4, C17 (1971).

14. D. S. Mather and L. F. Pain, AWRE 047/69 (1969).

15. John D. Anderson, private communication 1965, and Anderson et a1., Phys. Rev. Lett. 15, 66 (1965). 


\title{
SUMMARY DOCUMENTATION FOR ${ }^{3}$ He
}

\author{
by \\ L. Stewart \\ Los Alamos Scientific Laboratory \\ Los Alamos, New Mexico
}

\section{SUMMARY}

The ${ }^{3} \mathrm{He}$ evaluation for ENDF/B-V (MAT=1146) was carried over intact from Version IV. The evaluated data cover the energy range $10^{-5} \mathrm{eV}$ to $20 \mathrm{MeV}$, and documentation for the standards portion of the data is given in LA-6518-MS (1976).

\section{STANDARDS DATA}

The ${ }^{3} \mathrm{He}(\mathrm{n}, \mathrm{p}) \mathrm{T}$ cross section ( $M F=3 ; M T=103$ ) is recognized as a standard in the neutron energy range from thermal to $1 \mathrm{MeV}$. The present evaluation was performed in 1968 and accepted by the CSEWG Standards Subcomittee for the ENDF/B-III file in 1971. No changes have been recommended for this file; therefore, the present evaluation was carried over from both Versions III and IV of $\mathrm{ENDF} / \mathrm{B}$.

The thermal cross section of 5327 b was derived from precise measurements by Als-Nielsen and Dietrich ${ }^{2}$ of the total cross section up to an energy of 11

eV. No experimental measurements on the ${ }^{3} \mathrm{He}(n, p)$ reaction are available below $\sim 5 \mathrm{keV}$, and the cross section was assumed to follow $1 / \mathrm{v}$ up to $1.7 \mathrm{keV}$. The evaluation is compared with the available data below $10 \mathrm{keV}$ in Fig. 1 . For convenience, the inset includes tabular values of the elastic, $(n, p)$ and total cross sections at a few energies up to $1 \mathrm{keV}$.

Up to $10 \mathrm{keV}$, the evaluation is a reasonable representation of the 1966 results of Gibbons and Macklin ${ }^{3}$ and an average of their crosis sections measurnd in $1963 .{ }^{4}$ These experiments, which extend to $100 \mathrm{keV}$, are compared with ENDF/B-V in Fig. 2.

From $100 \mathrm{keV}$ to $1 \mathrm{MeV}$, additional experiments are available. The evaluation is heavily weighted by the data of Refs. 3 and 4 and the cross sections of Perry et $21 .^{5}$ as given in Fig. 3. Note that these three measurements are in guod agreement among themselves but are higher than the measurements of Batchelor et $a 1 .{ }^{6}$ and of Sayres et $a 1 .{ }^{7}$ On the other hand, Sayres et al. measure an elastic cross section much higher than reported by Seagrave et al. ${ }^{\circ}$ (noted on the same figure).

In 1970 , Costello et al. ${ }^{9}$ measured the $(n, p)$ cross section from $300 \mathrm{keV}$ to $1 \mathrm{MeV}$ and obtained essentially a constant value of $900 \mathrm{mb}$ over this energy range. Agreement of the Costello data with this evaluation above $500 \mathrm{keV}$ is excellent, although from 300 to $400 \mathrm{keV}$, their measurements are more than $10 \%$ lower than ENDF/B-V. 
Finally, Lopez et al. ${ }^{10}$ measured the relative ratio of the counting rates between ${ }^{3} \mathrm{He}$ and $\mathrm{BF}_{3}$ proportional counters from $218 \mathrm{eV}$ to $521 \mathrm{keV}$. To provide a comparison between these two standard cross sections, the Lopez ratios were normalized at $218 \mathrm{eV}$ to the Version IV ratios. Then, by using the present evaluation for the ${ }^{3} \mathrm{He}(n, p)$ cross section to convert the Lopez ratio measurements to ${ }^{10} \mathrm{~B}$ cross sections, reasonable agreement with Version $\mathrm{V}{ }^{10} \mathrm{~B}(n, \alpha)$ is obtained. It should be noted, however, that the energy points are too sparse above a few $\mathrm{keV}$ to reproduce the structure observed in $10 \mathrm{~B}$.

Although the thernal $(n, p)$ cross section is known to better than $1 \%$, the energy at which this cross section deviates from $1 / V$ is not well established. It should also be emphasized that experiments have not been carried out from $11 \mathrm{eV}$ to a few keV, thereby placing severe restrictions upon the accuracy accompanying the use of the ${ }^{3} \mathrm{He}(\mathrm{n}, \mathrm{p}) \mathrm{T}$ cross-section standard. The $10 \%$ error estimates on the ORNL experimental data are directly related to the uncertainties in the analysis of the target samples employed. Certainly, further absolute measurements are needed on this cross-section standard, especially above $\sim 100 \mathrm{eV}$.

\section{ENDF/B-V FILES}

File 1. General Information

MT=451. Descriptive data.

File 2. Resonance Parameters

MT: 151 . Scattering length $=0.2821 \mathrm{E}-12 \mathrm{~cm}$.

File 3. Neutron Cross Sections

MT $=1$. Total Cross Sections

From $0.00001 \mathrm{eV}$ to $10.8 \mathrm{keV}$ MT1 taken as sum MT2 + MT103. From

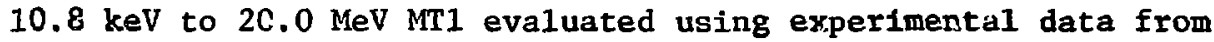
Ref. 11 .

MT=2 Elastic Scattering Cross Sections

From $0.00001 \mathrm{eV}$ to $10.8 \mathrm{keV}$ MT2 taken as constant $=1.0 \mathrm{~b}$. From $10.8 \mathrm{keV}$ to $20.0 \mathrm{MeV}$ MT2=MT1-MT103-MT104 with experimental data from Refs. 7 and 8 as checks. Note that two reactions are missing from the evaluation, namely, $\left(n, n^{\prime} p\right)$ and $(n, 2 n 2 p)$. Experimental data at $15 \mathrm{MeV}$ indicate non-zero cross sections for these reactions. In the present evaluation, these reactions are simply absorbed in $\mathrm{MT}=2$.

$M T=3$. $(n, p)$ Cross Section

Standards reaction - see Sec. II above.

MT=104. (n,d) Cross Sections

Threshold $=4.3614 \mathrm{MeV}, \mathrm{Q}=-3.2684 \mathrm{MeV}$. Evaluation from a detailed balance calculation (Ref. 2) and experimental data (Ref. 7). 
$M T=251$. Average Value of Cosine of Elastic Scattering Angle, Laboratory System.

Obtained from data $M F=4, M T=2$.

MT $=252$. Values of Average Logarithmic Energy Decrement

Obtained from data $\mathrm{MF}=4, \mathrm{MT}=2$.

MT=253. Values of Gamma

Obtained from data $\mathrm{MF}=4, \mathrm{MT}=2$.

Filé 4. Neutron Angular Distributions

MT=2. Angular Distribution of Secondary Neutrons From Elastic Scattering.

Evaluated from experimental data from Refs. 7, 8, 11-14 covering incident energies as follows:

INCIDENT ENERGY

$\begin{array}{rrr}1 . \mathrm{E}-5 \mathrm{eV} & \text { (Isotropic) } \\ 0.5 \mathrm{MeV} & \text { (Isotropic) } \\ 1.0 \mathrm{MeV} & 8 & \\ 2.0 \mathrm{MeV} & 8 & \\ 2.6 \mathrm{MeV} & 11 & \\ 3.5 \mathrm{MeV} & 8 & \\ 5.0 \mathrm{MeV} & 11 & \\ 6.0 \mathrm{MeV} & 8,12 \text { (from p+t elastic scattering) } \\ 8.0 \mathrm{MeV} & 7,12 \text { (from p+t elastic scattering) } \\ 14.5 \mathrm{MeV} & 12,13 \text { (from p+t elastic scattering) } \\ 17.5 \mathrm{MeV} & 7 \text { (from p+t elastic scattering) } \\ 20.0 \mathrm{MeV} & 11 \text { (from }\end{array}$

\section{REFERENCES}

1. This evaluation was translated by $R$. J. LaBauve into the ENDF/B format for Version III.

2. J. Als-Nielsen and 0 . Dietrich, "Slow Neutron Cross Sections for $\mathrm{He}^{3}, \mathrm{~B}$, and Au," Phys. Rev. 133, B 925 (1964).

3. J. H. Gibbons and R. L. Macklin, "Total Neutron Yields from Light Elements under Proton and Alpha Bombardment," Phys. Rev. 114, 571 (1959).

4. R. L. Macklin and J. H. Gibbons, Proceedings of the International Conference on the Study of Nuclear Structure with Neutrons, Antwerp, 19-23 July 1965 (North-Holland Publishing Co., 1966), p. 498.

5. J. E. Perry, Ir., E. Haddad, R. L. Henke1, G. A. Jarvis, and R. K. Smith, private communication 1960 . 
6. R. Batchelor, R. Aves, and T. H. R. Sikyrme, "Helium-3 Filled Proportional Counter for Neutron Spectroscopy," Rev. Sci. Instr. 26, 1037 (1955).

7. A. R. Sayres, K. W. Jones, and C. S. Wu, "Interaction of Neutrons with $\mathrm{He}^{3}, "$ Phys. Rev. 122, 1853 (1961).

8. J. D. Seagrave, L. Cranberg, and J. E. Simnons, "Elastic Scattering of Fast Neutrons by Tritium and He"," Phys. Rev, 119, 1981 (1960).

9. D. G. Costello, S. J. Friesenhahn, and W. M. Lopez, " ${ }^{3} \mathrm{He}(\mathrm{n}, \mathrm{p}) \mathrm{T}$ Cross Section from 0.3 to $1.16 \mathrm{MeV}, "$ Nucl. Sci. Eng. 39, 409 (1970).

10. W. M. Lopez, M. P. Fricke, D. G. Costello, and S. J. Friesenhahn, "Neutron Capture Cross Sections of Tungsten and Rhenium," Gulf General Atomic, Inc. report GA-8835.

11. Los Alamos Physics and Cryogenics Groups, Nuc1. Phys. 12, 291 (1959).

12. J. E. Brolley, Jr., T. M. Putnam, L. Rosen, and L. Stewart, Phys. Rev。 159, 777 (1967).

13. B. Antolkovic, G. Paic, P. Tomas, atd R. Rendic, Phys. Rev. 159,777 (1967).

14. L. Rosen and W. Leland, Private communication (1967).

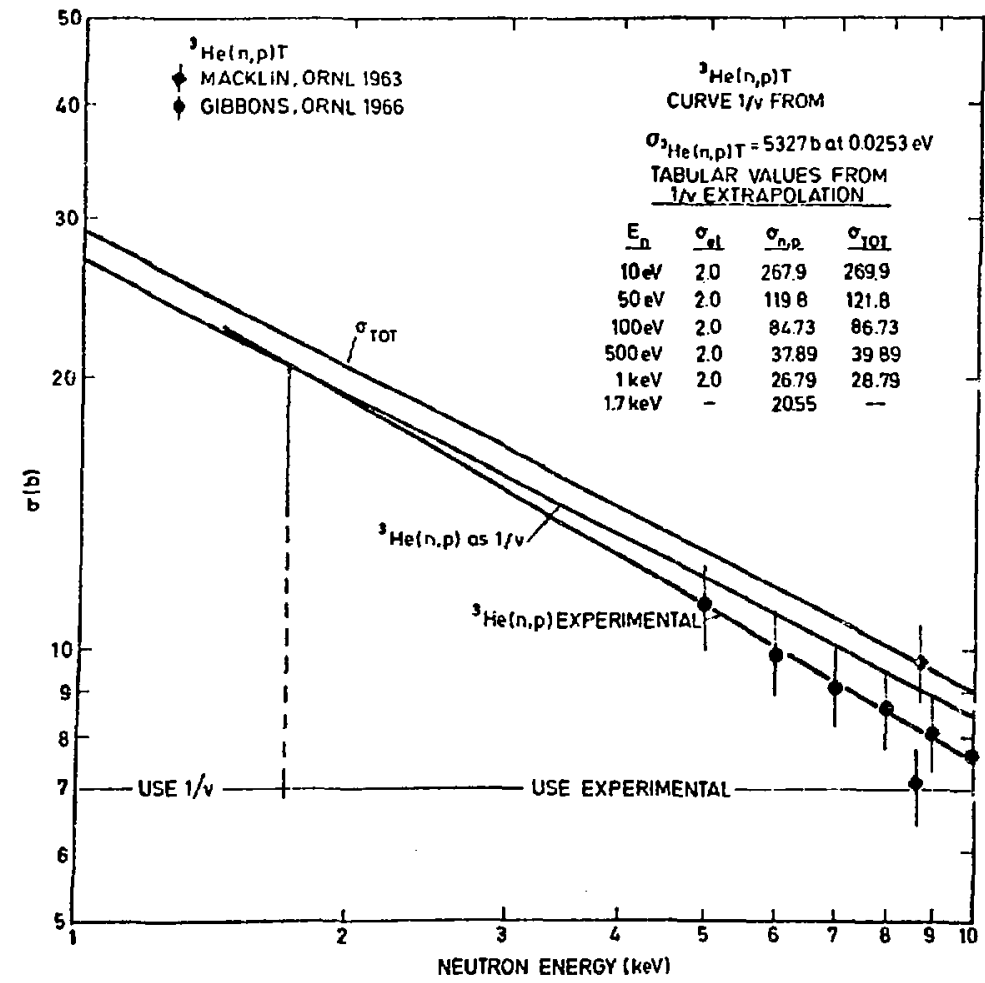

Fig. 1.

The $(n, p)$ and total cross sections for ${ }^{3} \mathrm{He}$ from 1 to $10 \mathrm{keV}$. The curve drawn through the experimental points deviates from $1 / \mathrm{V}$ at $1.7 \mathrm{keV}$. 


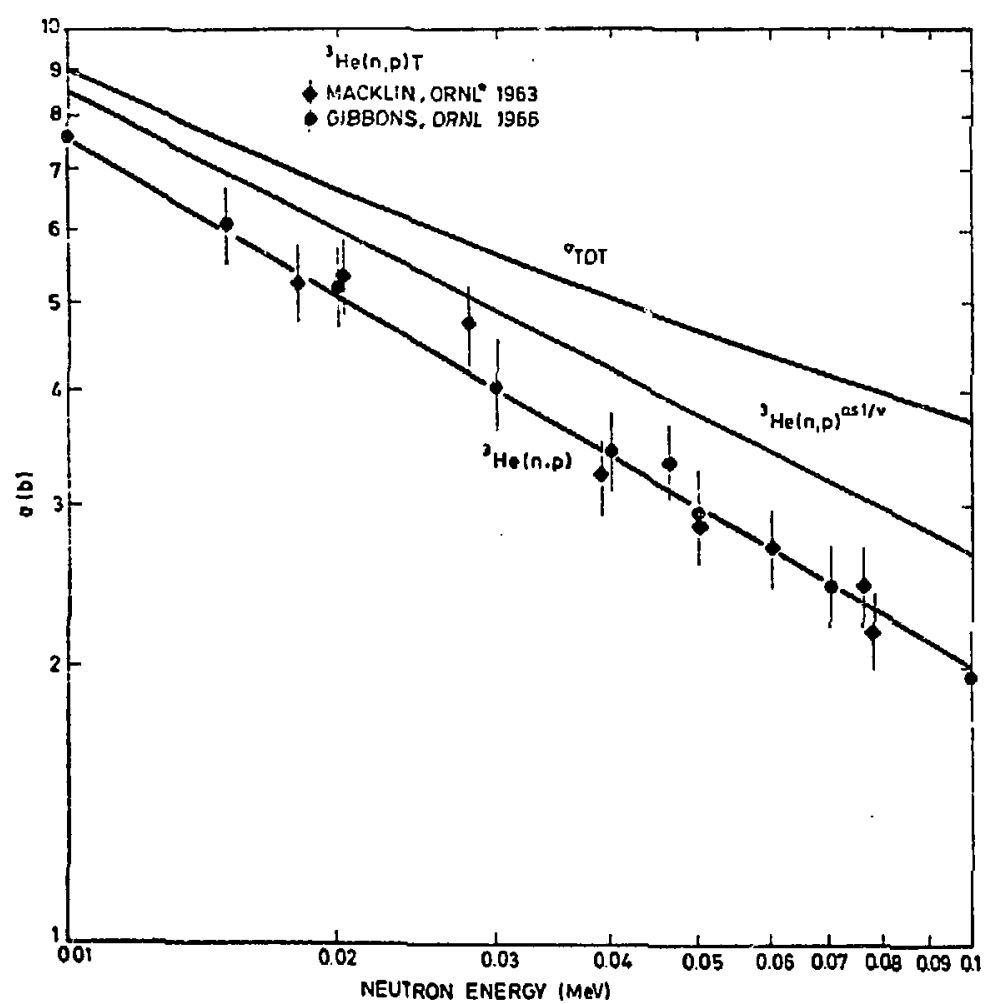

Fig. 2 .

The $(n, p)$ and total cross sections for ${ }^{3}$ He from 10 to $100 \mathrm{keV}$.

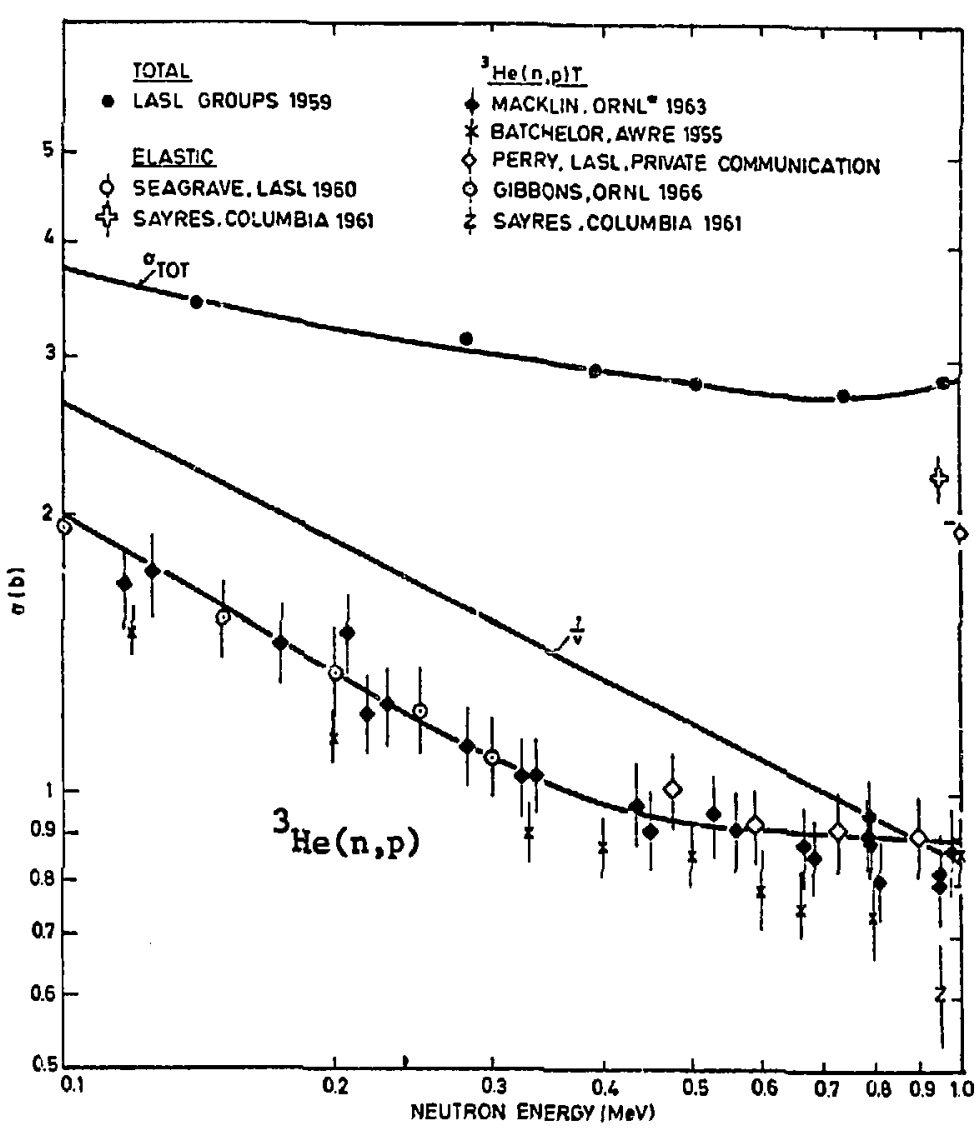

Fig. 3 .

The $(n, p)$, elastic, and total cross sections for $3 \mathrm{He}$ from $100 \mathrm{keV}$ to $1 \mathrm{MeV}$. The Costello data 9 have been omitted for the sake of clarity. 
SUMMARY DOCUMENTATION FOR ${ }^{4}$ He

by

G. M. Hale, R. A. Nisley, and P. G. Young

Los Alamos Scientific Laboratory

Los Alamos, New Mexico

\section{SUMMARY}

The ENDF/B-V evaluation for ${ }^{4} \mathrm{He}$ (MAT 1270) is the same as Version IV except for minor format changes. The evaluation covers the energy range $10^{-5} \mathrm{eV}$ to 20 $\mathrm{MeV}$ and is based at all energies on an extensive R-matrix analysis, which is described in NEANDC (j) 38L (reference Dc75). By making use of the charge symmetry of nuclear forces, $\mathrm{p}^{4} \mathrm{He}$ data were included in the analysis along with the available $n-{ }^{4} \mathrm{He}$ measurements of cross sections, angular distributions, and polarizations. Because of the extent of the data base used and the careful analysis it was given, the cross sections and angular distributions are thought to be accurate to about $\pm 2 \%$ at all energies. As all gamma-ray production cross sections are essentially zero for ${ }^{4} \mathrm{He}$, gamma ray files (MF=12-16) are deliberately excluded from the evaluation.

II. ENDF/B-V FILES

File 1. General Information

MT=451. Descriptive data.

File 2. Resonance Parameters

$\mathrm{MT}=151$. Effective scattering radius $=0.24579 \times 10^{-12} \mathrm{~cm}$.

Resonance parameters not given.

File 3. Neutron Cross Sections

The $2200 \mathrm{~m} / \mathrm{s}$ cross sections are as follows:

$$
\begin{array}{ll}
\text { MT }=1 & \text { Sigma }=0.75916 b \\
M T=2 & \text { Sigma }=0.75916 b
\end{array}
$$

MT=1. Total Cross Section

See discussion under $\mathrm{MT}=2$ below. 


\section{MT=2. Elastic Scattering Cross Section}

Although the only reaction possible for neutrons incident on ${ }^{4} \mathrm{He}$ below $20 \mathrm{MeV}$ is elastic scattering, the majority of the $n-{ }^{4} \mathrm{He}$ data is rather imprecise. In order to overcome this problem, an R-matrix. analysis was performed with a data set which included not only the $n-{ }^{4} \mathrm{He}$ data but also very precise $\mathrm{p}-{ }^{4} \mathrm{He}$ data. All the avallable $n-{ }^{4}$ He and $p^{-4} \mathrm{He}$ data belon $20 \mathrm{MeV}$ were considered in . e analysis. Since the previous evaluation was completed in 1968, several $\mathrm{n}-{ }^{4} \mathrm{He}$ elastic scattering measurements have been done. The most significant of these are the low energy neutron cross sections of Rorer (Ro69), the RPI total cross section measurement (Go73), which cover the range $E_{\mathrm{n}}=0.7-30 \mathrm{MeV}$, and the relative angular distributions of Morgan (Mo68). A complete list of references for the $n^{-4}$ lie data used is given below. The $\mathbf{p}-{ }^{4} \mathrm{He}$ data was selected to satisfy very stringent statistical criteria and we believe the possible errors of the predicted values for the $\mathrm{p}-{ }^{4} \mathrm{He}$ scattering to be less than $1.0 \%$. A simple model for the charge differences between the $n-{ }^{4} \mathrm{He}$ and $\mathrm{p}-{ }^{4} \mathrm{He}$ systems was assumed and the $n-{ }^{4} \mathrm{He}$ and $\mathrm{p}^{-4} \mathrm{He}$ data sets were simultaneous $1 \mathrm{y}$ analyzed. The values of the cross sections and angular distributions contained in Files 3 and 4 are probably accurate to within $2.0 \%$.

Comparisons of the evaluated and experimental total cross section data are given in Figs. 1-4, and the elastic angular distribution data are included in Figs. 5-11. The neutron polarization measements that were included in the R-matrix analysis are also shown in Figs. 12 and 13.

\section{File 4. Neutron Angular Distributions}

MT=2. Elastic Scattering Angular Distributions.

Obtained from the R-matrix analysis described above under $M F=3$, $M T=2$. Legendre polynomial representation used in the $\mathrm{cm}$ system. See Figs. 5-11 for data.

\section{REFERENCES}

Au62 S. M. Austin et al., Phys. Rev. 126, 1532 (1962).

Br72 W. B. Broste et al., Phys. Rev. C5, 761 (1972).

Bu66 F. W. Busser et al., Nucl. Phys. 88, 593 (1966).

Cr72 D. S. Cramer and L. Cranberg, Nucl. Phys. A180, 273 (1972).

Do75 D. C. Dodder, G. M. Hale, R. A. Nisley, K. Witte, and P. G. Young, Proc. of the EANDC Topical Discussion on "Critique of Nuclear Models and Their Validity in the Evaluation of Nuclear Data," Mar. 1974, p. 1. (Published in NEANDC (J) 38L (1975).

Fa63 U. Faso1i and G. Zago, Nuovo Cimento 30, 1169 (1963).

Go73 C. A. Coulding et al., Nuc1. Phys. A215, 253 (1973).

Ho66 B. Hoop, Jr. and H. H. Barschall, Nucl. Phys. 83, 65 (1966).

Je66 R. W. Jewe11 et al., Phys. Rev, 142687 (1966). 
Ma63 T. H. May et a1., Nuc1. Phys. 45,17 (1963) (Rev. Mo68 and Sa68).

Mo68 G. L. Morgan and R. L. Walker, Phys. Rev. 168, 1114 (1968).

Ni71 A. Niiler et a1., Phys. Rev. C4, 36 (1971) (Rev. 9-72).

Ro69 D. C. Rorer et a1., Nucl. Phys. 133, 410 (1969).

Sa68 J. R. Sawers et al., Phys. Rev. 168,1102 (1968).

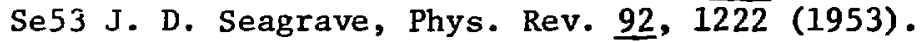

Sh55 D. F. Shaw, Proc. Phys. Soc. (London) 68, 43 (1955).

Sh64 R. E. Shamu and J. G. Jenkins, Phys. Rev. 135, B99 (1964).

Sm54 J. R. Smith, Phys. Rev. 95, 730 (1954).

St70 T. Stammbach et al., Phys. Rev. C2, 434 (1970).

Wh57 R. E. White and F. J. M. Farley, Nucl. Phys. 3,476 (1957).

Yo63 P. G. Young et a1., Aust. J. Phys. 16, 185 (1963). 


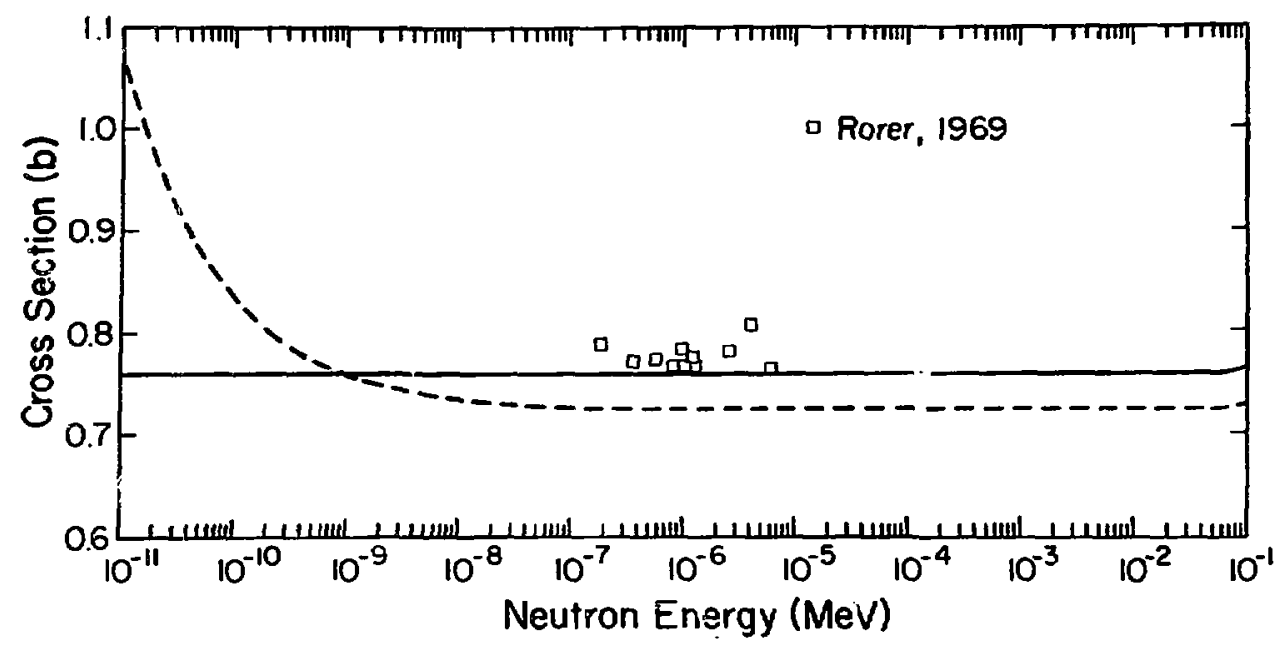

Fig. I.

Measured and evaluated $n-" \mathrm{Fo}$ total cross sections between $10^{-5} \mathrm{eV}$ and $100 \mathrm{keV}$. The solid curve is ENDF/B-V and the dashed curve is ENDF/B-III.

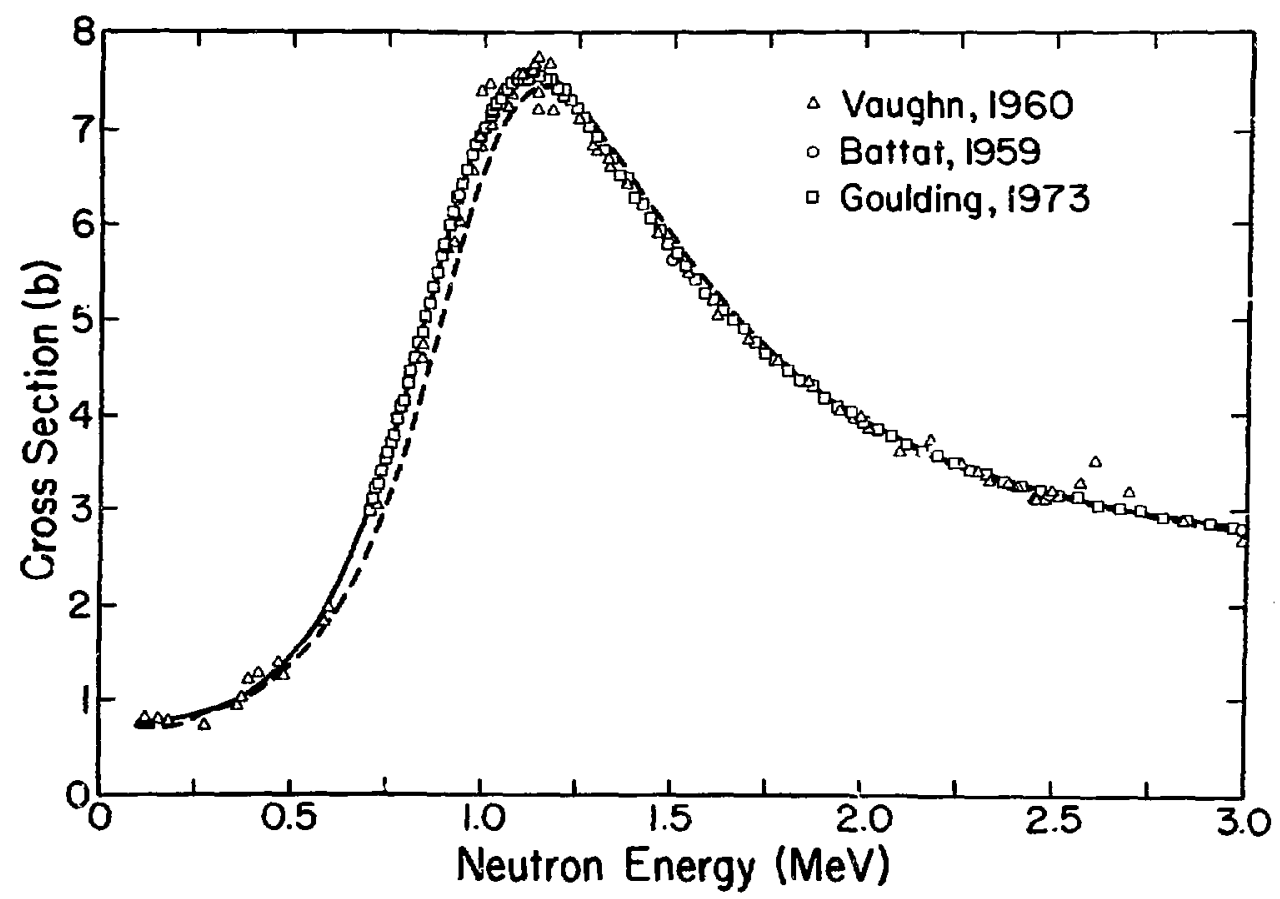

Fig. 2 .

Measured and evaluated $n-{ }^{4} \mathrm{He}$ total cross sections between 0 and $3 \mathrm{MeV}$. The solid curve is ENDF/B-V and the dashed curve is ENDF/B-III. 


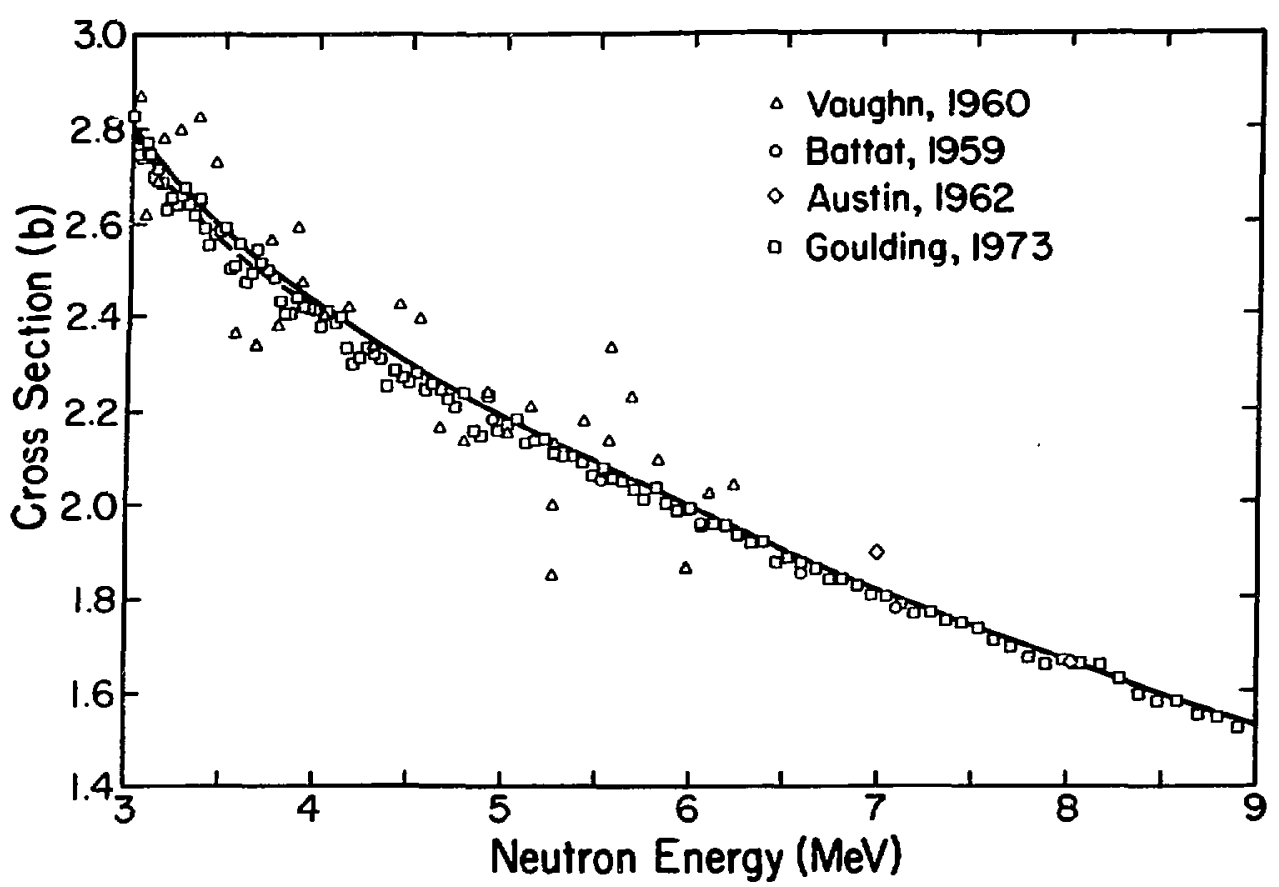

Fig. 3.

Measured and evaluated $n-{ }^{4} H e$ total cross sections between 3 and $9 \mathrm{MeV}$. The solid curve is ENDF/B-V and the dashed curve is $\mathrm{ENDF} / \mathrm{B}-\mathrm{III}$.

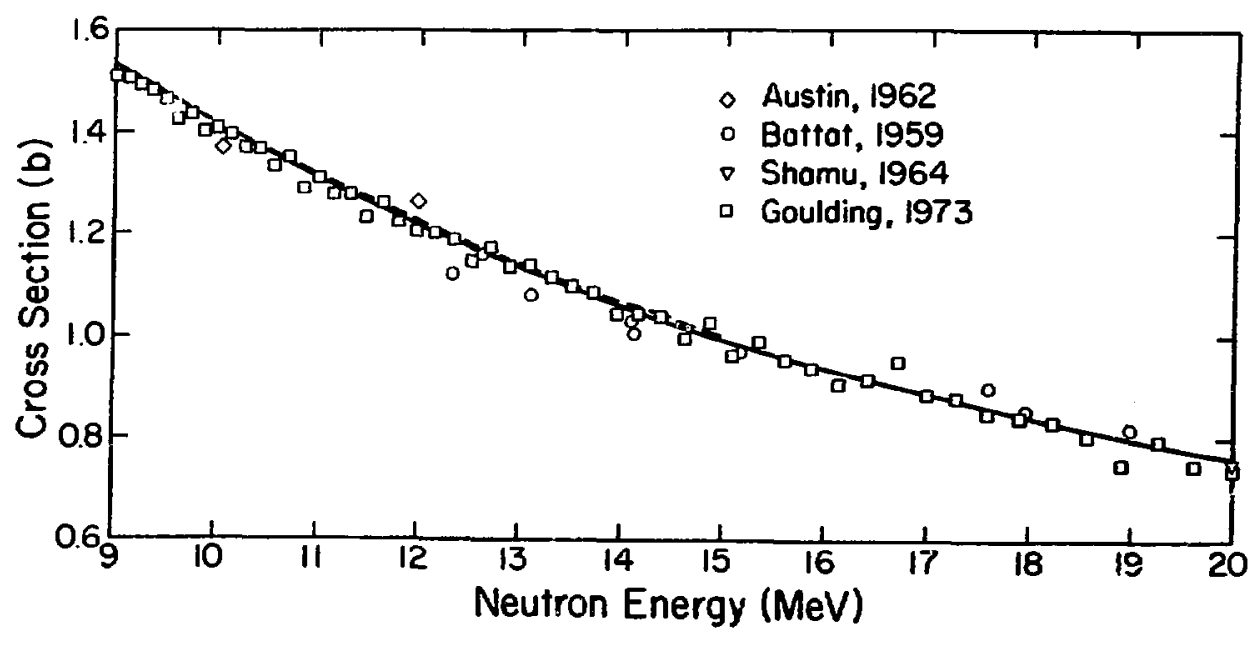

Fig. 4.

Measured and evaluated $n-4$ He total cross sections between 9 and $20 \mathrm{MeV}$. The solid curve is ENDF/B-V and the dashed curve is ENDF/B-III. 

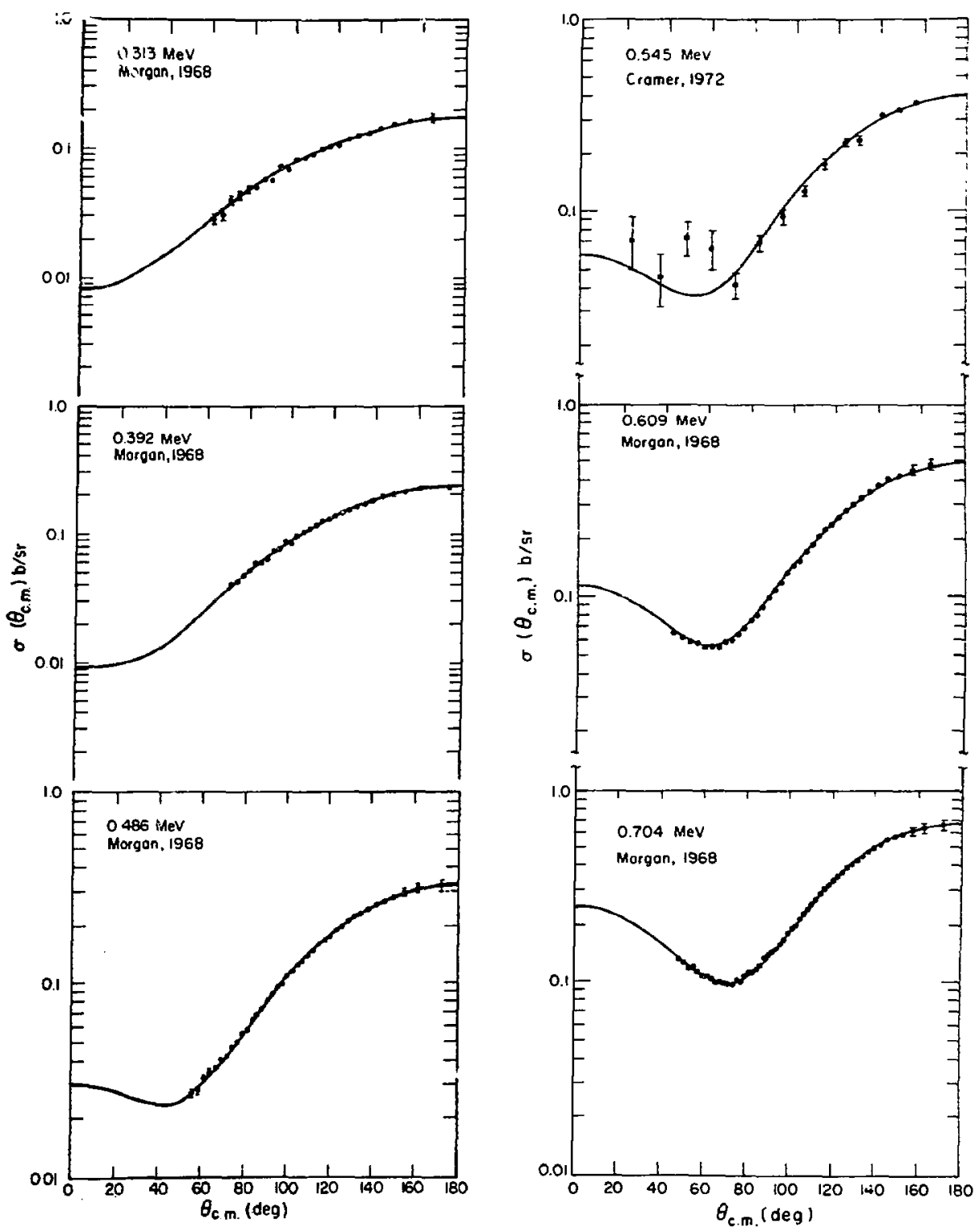

Fig. 5.

Measured and evaluated $n-{ }^{4} \mathrm{He}$ differential elastic angular distribution for incident neutron energies between 0.313 and $0.704 \mathrm{MeV}$. 

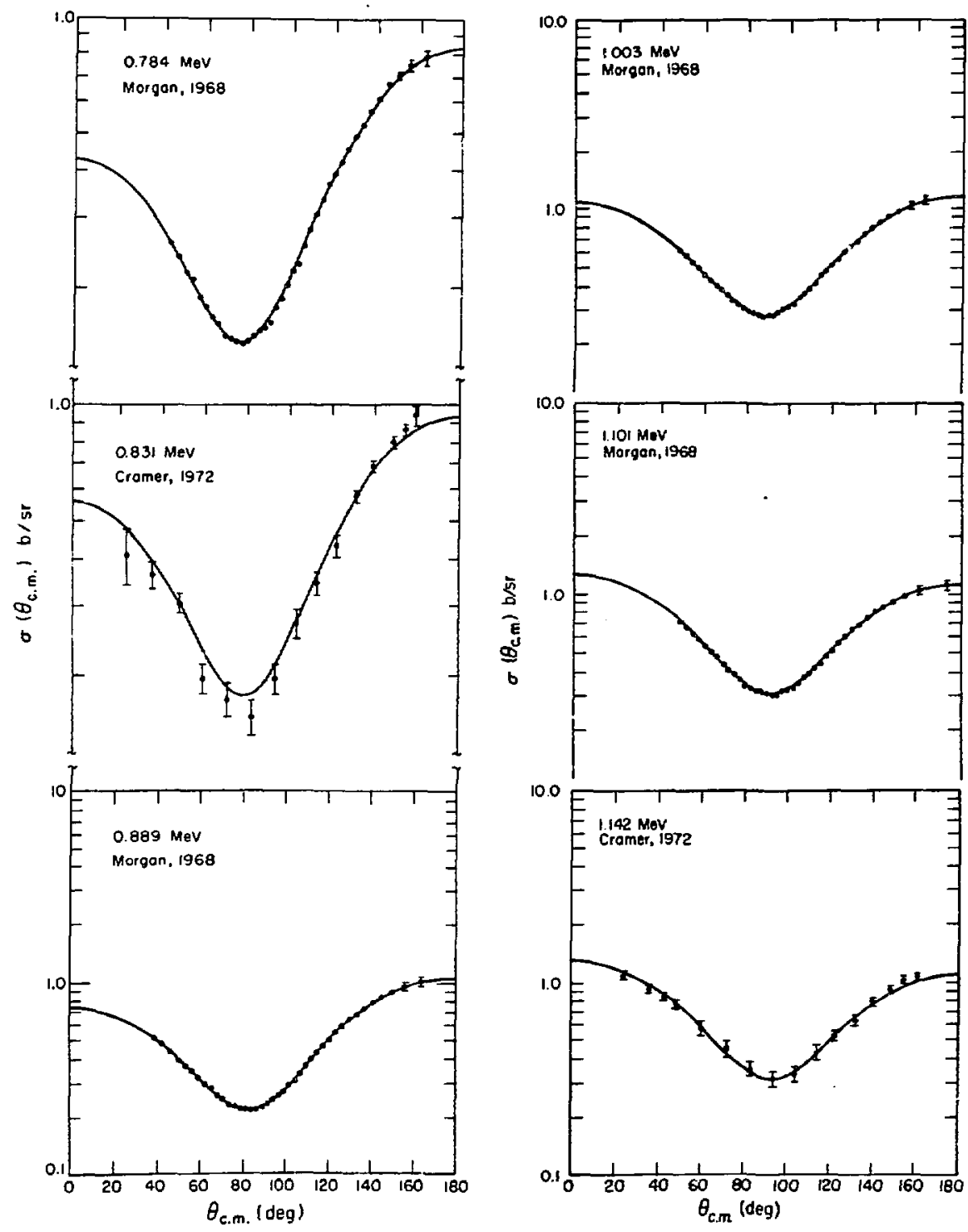

Fig. 6 .

Measured and evaluated $n-{ }^{4}$ He differential elastic angular distribution for incident neutron energies between 0.784 and $1.142 \mathrm{MeV}$. 

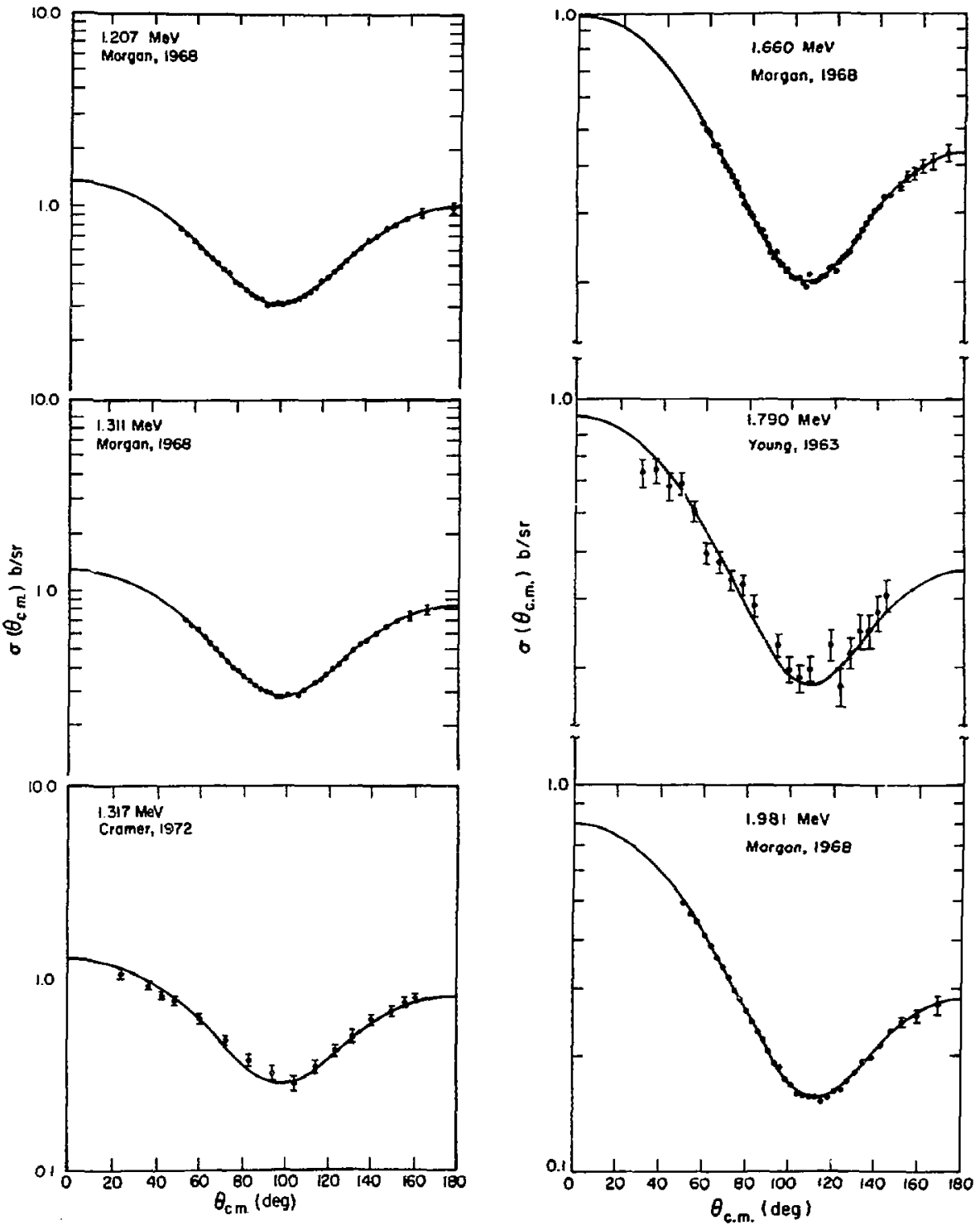

Fig. 7.

Measured and evaluated $n-{ }^{4} \mathrm{He}$ differential elastic angular distribution for incident neutron energies between 1.207 and $1.981 \mathrm{MeV}$. 

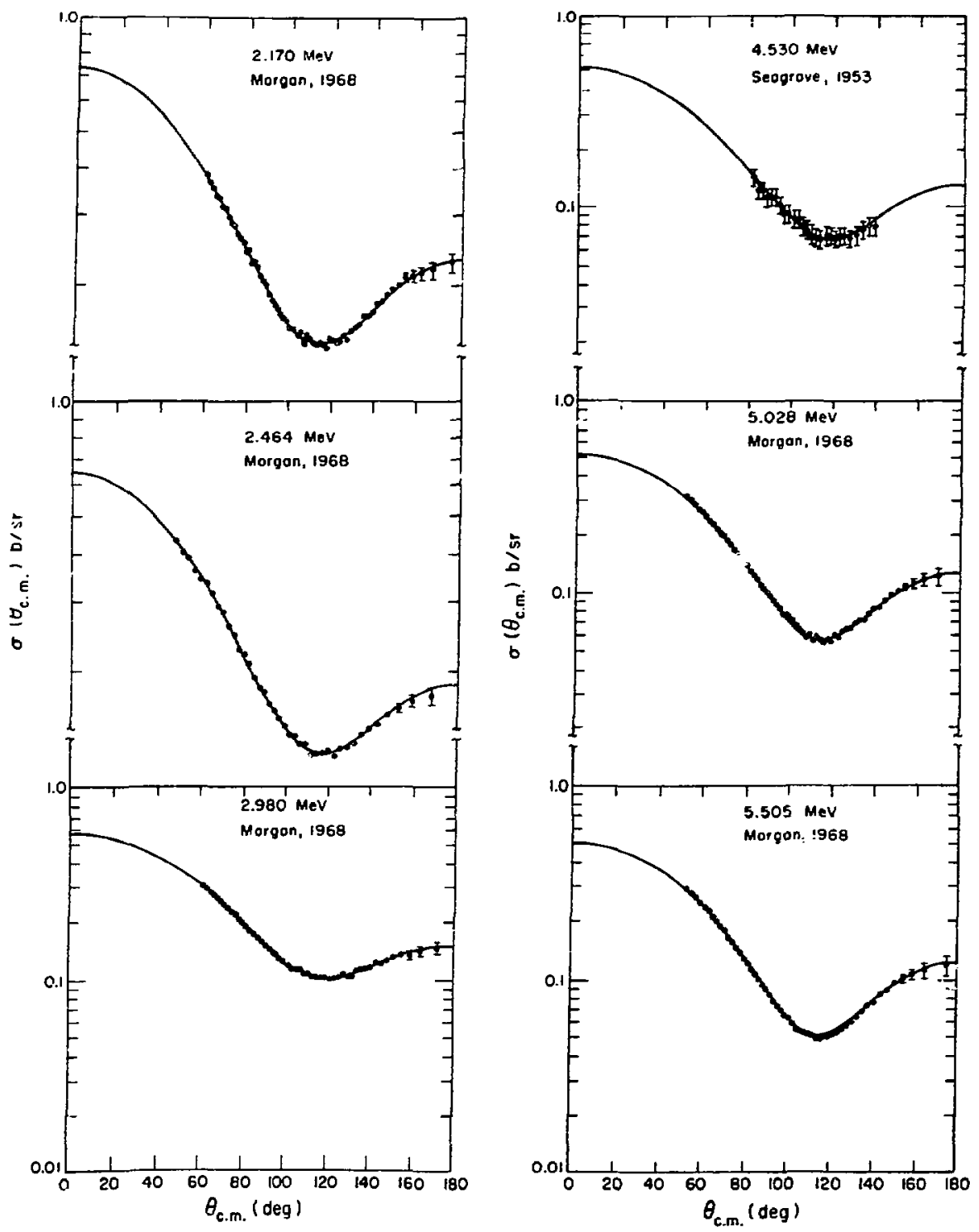

Fig. 8 .

Measured and evaluated $n-{ }^{4} H e$ differential elastic angular distribution for incident neutron energies between 2.170 and $5.505 \mathrm{MeV}$. 

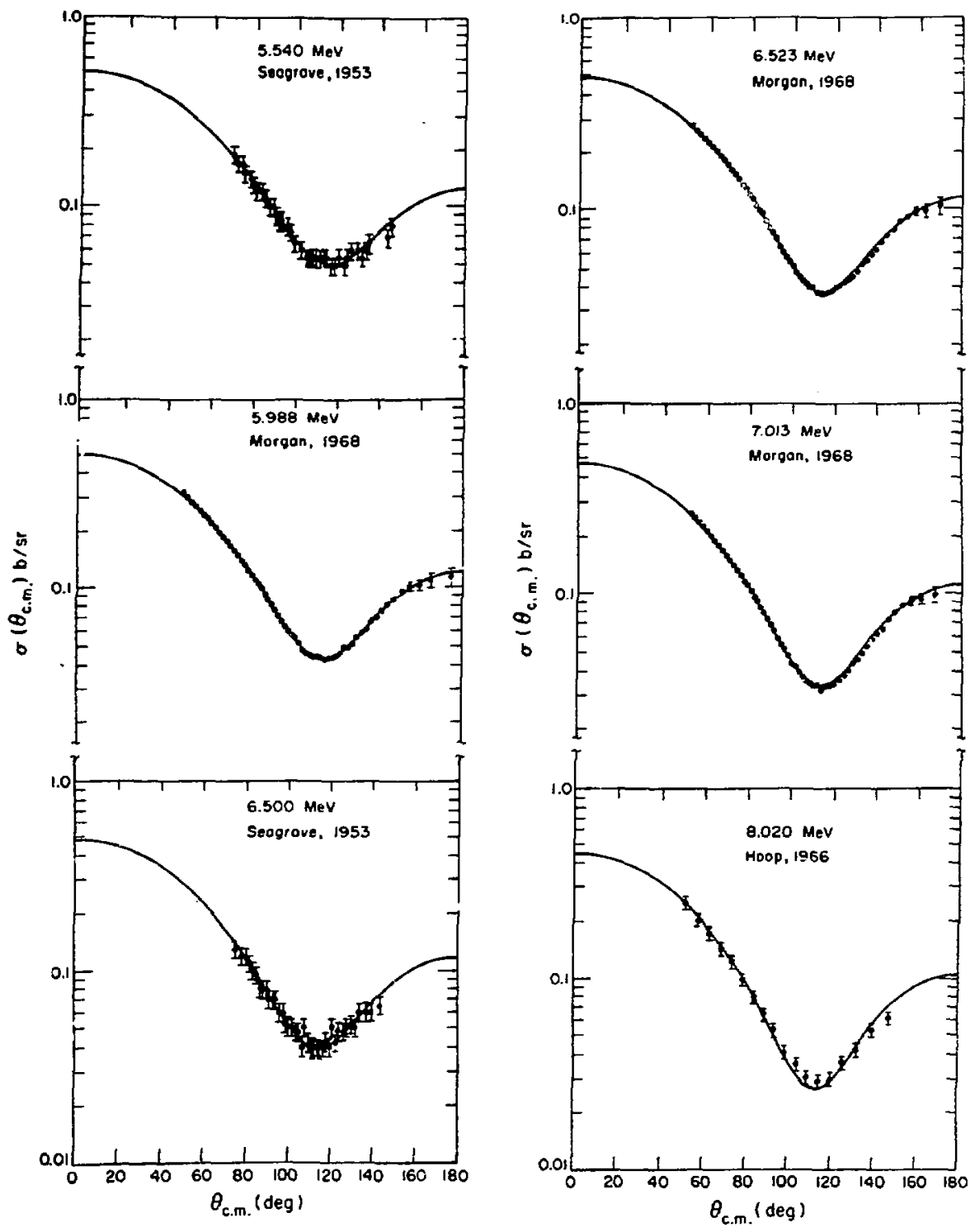

Fig. 9.

Measured and evaluated $n-{ }^{4}$ He differential elastic angular distribution for incident neutron energies between 5.54 and $8.08 \mathrm{MeV}$. 

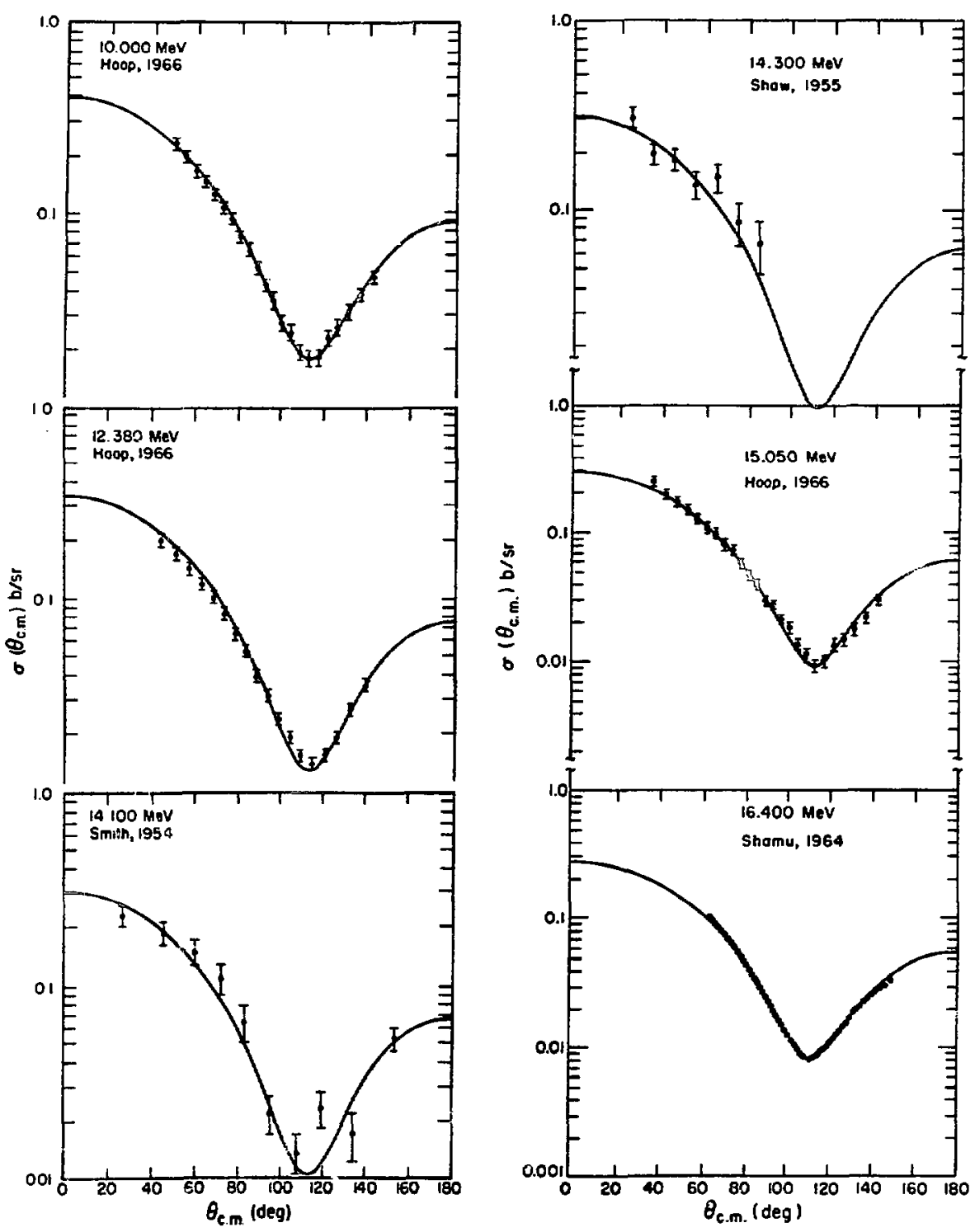

Fig. 10 .

Mealsured and evaluated $n-{ }^{4} H e$ differentlal elastic angular distribution for incident neutron energies between 10.0 and $16.4 \mathrm{MeV}$. 


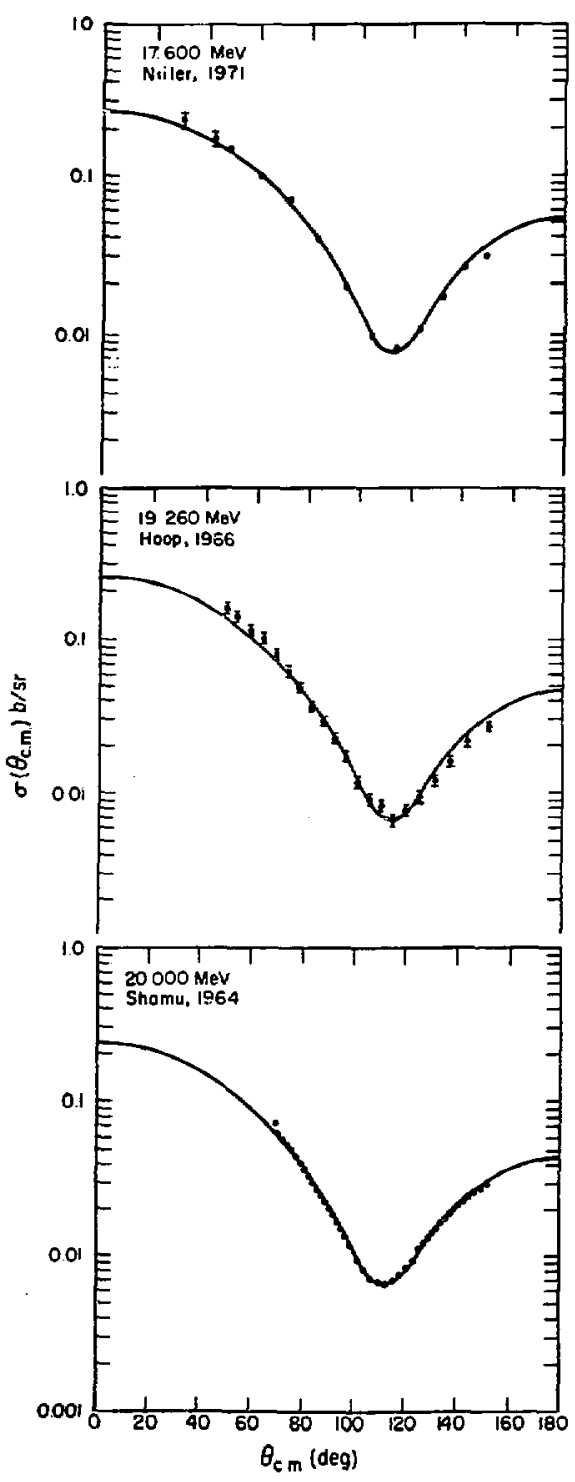

Fig. 11.

Measured and evaluated $n-{ }^{4} \mathrm{He}$ differential elastic angular distribution for incident neutron energies between 17.6 and $20.0 \mathrm{MeV}$. 

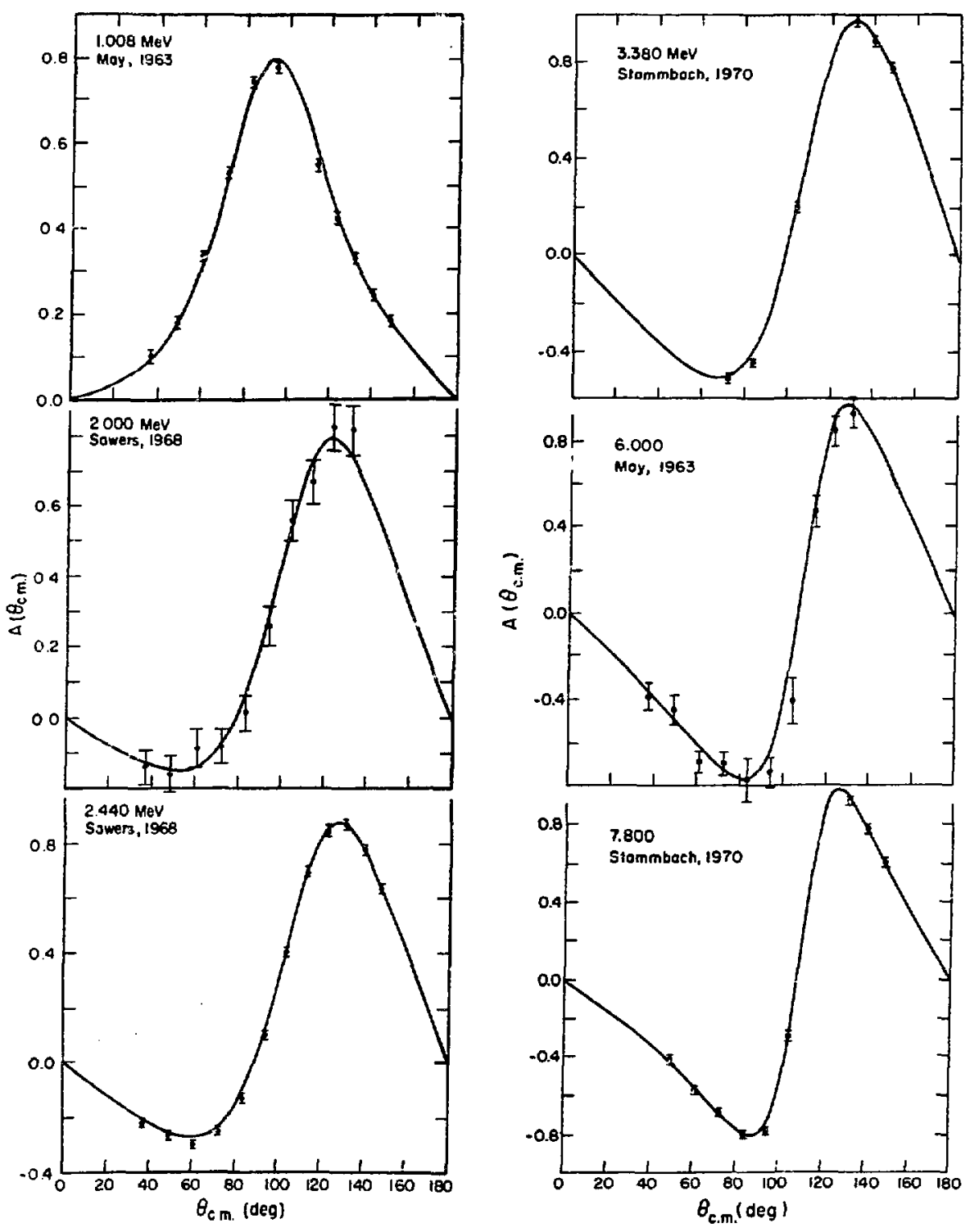

Fig. 12 .

${ }^{4} \mathrm{He}(n, n){ }^{4} \mathrm{He}$ neutron polarizations for incident energies between 1.008 and $7.8 \mathrm{MeV}$. 

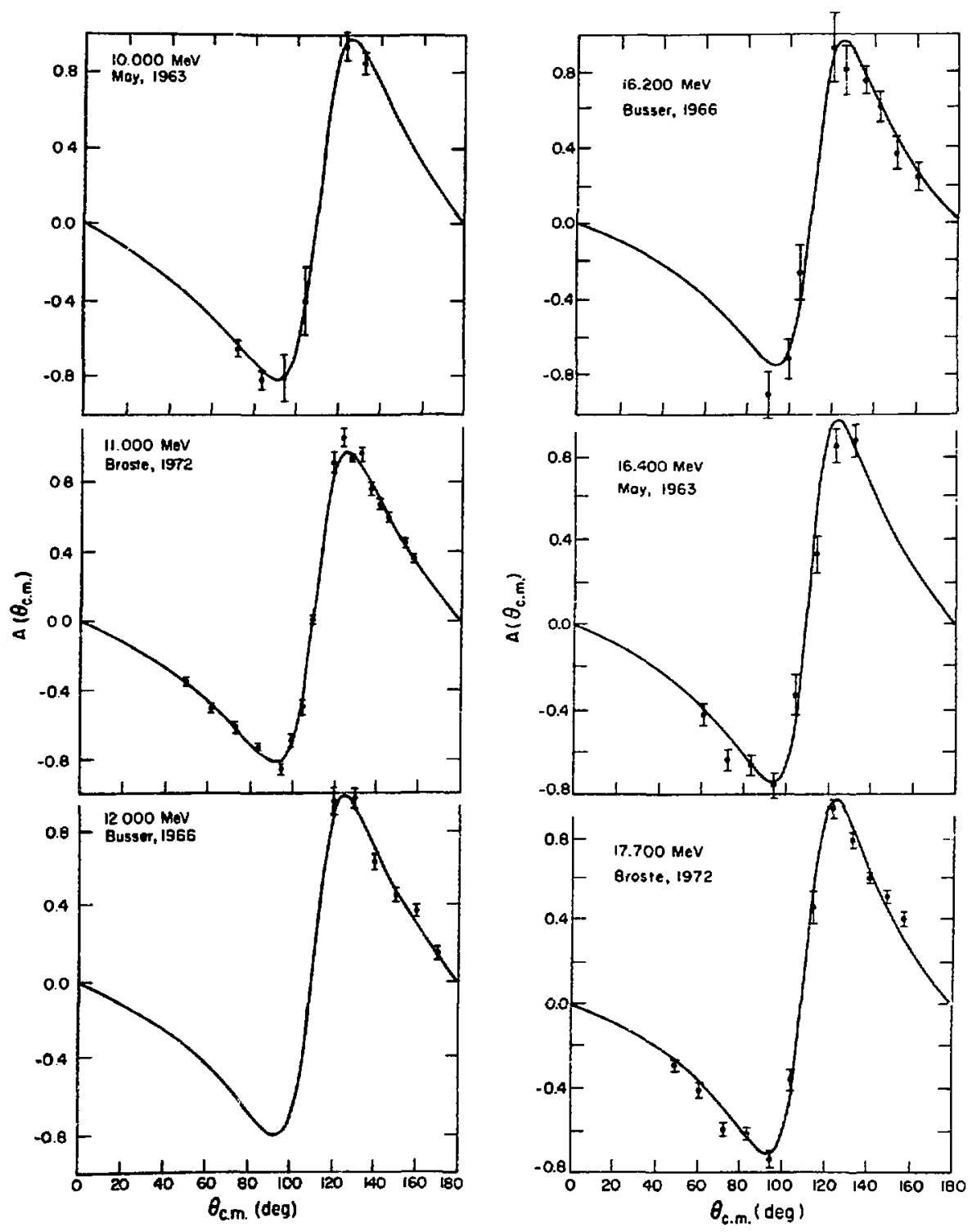

Fig. 13.

${ }^{4} \mathrm{He}(n, n){ }^{4} \mathrm{He}$ neutron polarizations for incident energies between 10.0 and $17.7 \mathrm{MeV}$. 
SUMMARY DOCUMENTATION FOR ${ }^{6} \mathrm{Li}$

by

G. M. Hale, L. Stewart, and P. G. Young

Los Alamos Scientific Laboratory

Los Alamos, New Mexico

\section{SUMMARY}

The previous evaluation for ${ }^{6} \mathrm{Li}$ was extensively revised for Version $\mathrm{V}$ of ENDF/B (MAT 1303). All major cross-section files except radiative capture were updated. A new R-matrix analysis including recent experimental results was performed up to a neutron energy of $1 \mathrm{MeV}$, which includes the standards region for the ${ }^{6} \mathrm{Li}(n, t){ }^{4} \mathrm{He}$ reaction. Extensive revisions were made in the MeV region to include a more precise representation of the $\left(n, n^{\prime} d\right)$ reaction. In the new representation, the $\left(n, n^{\prime} d\right)$ cross section is grouped into ${ }^{6} \mathrm{Li}$ excitation energy bins, which preserves the kinematic energy-angle relationships in the emitted neutron spectra. Finally, correlated error data were added up to a neutron energy of $1 \mathrm{MeV}$, triton angular distributions from the ${ }^{6} \mathrm{Li}(\mathrm{n}, \mathrm{t}){ }^{4} \mathrm{He}$ reaction were included below $1 \mathrm{MeV}$, and radioactive decay data were added to Files 8 and 9 . Except for the covariance and $(n, t)$ angular distribution files, the evaluation covers the neutron energy range of $10^{-5} \mathrm{eV}$ to $20 \mathrm{MeV}$.

\section{STANDARDS DATA}

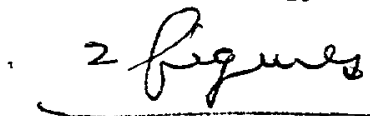

The ${ }^{6} \mathrm{Li}(\mathrm{n}, \alpha)$ cross section is regarded as a standard below $E_{n}=100 \mathrm{keV}$. The Version $V$ cross sections for ${ }^{6} \mathrm{Li}$ below $1 \mathrm{MeV}$ were obtained from multichannel, multilevel R-matrix analyses of reactions in the ${ }^{7} \mathrm{Li}$ system, similar to those from which the Version IV evaluation were taken. New data have become available since Version IV was released and most of this new experimental information has been incorporated into the Version $\mathrm{V}$ analysis.

For Version IV, the ${ }^{6} \mathrm{Li}(\mathrm{n}, \alpha)$ cross section was determined mainly by fitting the Harwell total cross section (reference 3 below), since this was presumably the most accurately known data included in the analysis. However, in addition to the Harwell total, the data base for the analysis included the shapes of the $n-{ }^{6} \mathrm{Li}$ elastic angular distributions and polarizations, ${ }^{6} \mathrm{Li}(\mathrm{n}, \alpha) \mathrm{T}$ angular distributions and integrated cross sections (normalized), and $t-\alpha$ elastic angular distributions.

Since the time of the Version IV analysis, new data have become available whose precision equals or betters that of the Harwell total cross section. The present analysis includes the following new measurements while retaining most of the data from the previous analysis: 
Measurement

$n-{ }^{6} L i \quad \sigma_{T}$

${ }^{6} \mathrm{~L} i(n, \alpha)$ integrated cross section

${ }^{4} \mathrm{He}(t, t){ }^{4} \mathrm{He}$ differential cross section

${ }^{4} \mathrm{He}(\vec{t}, t){ }^{4}$ He analyzing power

\begin{tabular}{|c|c|}
\hline References & $\begin{array}{c}\text { Approximate } \\
\text { Precision }\end{array}$ \\
\hline Harvey, ORNL ${ }^{4}$ & $0.5-1 \%$ \\
\hline Lamaze, NBS ${ }^{21}$ & $1-2 \%$ (relative) \\
\hline Jarmie, LASL ${ }^{35}$ & $0.4-1 \%$ \\
\hline Hardekopf, $\mathrm{LASL}^{36}$ & $1 \%$ \\
\hline
\end{tabular}

Fits to the $(n, \alpha)$ data included in the Version $V$ analysis are shown in Figs. 1 and 2. In Fig. 1, the data are plotted as $\sigma \cdot \sqrt{E_{n}}$; in both figures, the Version IV evaluation is represented by the dashed curves. The good agreement with Lamaze's new ${ }^{6} \mathrm{Li}(\mathrm{n}, \alpha)$ integrated cross section measurement ${ }^{2}{ }^{1}$ is particularly encouraging, since these are close to the values most consistent with the accurate new $t+\alpha$ measurements. ${ }^{35,36}$ on the other hand, a shape difference persists between the fit and measurements of the total cross section in the region of the precursor dip and at the peak of the 245-keV resonance. However, we feel that including these precise new data in the analysis has reduced the uncertainty of the new ${ }^{6} \mathrm{Li}(n, \alpha)$ cross section significantly (to the order of $3 \%$ ) over that of previous evaluations in the region of the resonance.

\section{ENDF/B-V FILES}

File 1. General Information

$\mathrm{MT}=451$. Descriptive data.

\section{File 2. Resonance Parameters}

$M T=151$. Effective scattering radius $=0.23778 \times 10^{-12} \mathrm{~cm}$.

Resonance parameters not given.

File 3. Neutron Cross Sections

The $2200 \mathrm{~m} / \mathrm{s}$ cross sections are as follows:

$$
\begin{array}{llr}
M T=1 & \text { Sigma }=936.64 & \mathrm{~b} \\
\mathrm{MT}=2 & \text { Sigma }=0.71046 & \mathrm{~b} \\
\mathrm{MT}=102 & \text { Sigma }=0.03850 \mathrm{~b} \\
\mathrm{MT}=105 & \text { Sigma }=935.89 & \mathrm{~b}
\end{array}
$$

MT=1. Total Cross Section

Below $1 \mathrm{MeV}$, the values are taken from an R-matrix analysis by Hale, Dodder, Witte (described in Ref. 2) which takes into account data from all reactions possible in ${ }^{7} \mathrm{Li}$ up to $3 \mathrm{MeV}$ neutron energy. Total cross section data considered in this analysis were those of Refs. 3 and 4. Between 1 and $5 \mathrm{MeV}$, the total was taken to be the sum of MT=2, 4, 24, 102, 103, and 105, which generally follows the measurements of Refs. 5 and 6. Between 5 and $20 \mathrm{MeV}$, the total was determined by an average of the data of Refs. 6 and 7 which agrees with Ref. 8 
except at the lowest energy. In this region, the total exceeds the sum of the measured partial cross sections by as much as 200-300 mb. This difference was distributed between the elastic and total $\left(n, n^{\prime}\right) d$ cross sections.

\section{$\mathrm{MT}=2$. Elastic Cross Section}

Below $3 \mathrm{MeV}$, the values are taken from the R-matrix analysis cited for $M T=1$, which includes the $e^{i}$ astic measurements of Refs. 9 and 10. These calculations were nutched smoothly in the 3-5 MeV region to a curve which lies about $50 \mathrm{mb}$ above Batchelor (Ref. 26) between 5 and $7.5 \mathrm{MeV}$, and abuut 13\% above the data of Refs. 14, 27,28 , and 29 at 10 to $14 \mathrm{MeV}$.

$M T=4$. Inelastic Cross Section

Sum of $M T=51$ through $M T=81$.

$M T=24 . \quad(n, 2 n) \alpha$ Cross Section

Passes through the point of Mather and Pain (Ref. 11) at $14 \mathrm{MeV}$, taking into account the measurements of Ref. 12 .

$\mathrm{MT}=51,52,54-56,58-81 . \quad\left(\mathrm{n}, \mathrm{n}^{\prime}\right) \mathrm{d}$ Continuum Cross Sections

Represented by continuum-level contributions in ${ }^{6} \mathrm{~L} 1$, binned in $0.5-\mathrm{MeV}$ intervals. The energy-angle spectra are determined by a 3-body phase-space calculation, assuming isotropic center-ofmass distributions. At each energy, the sum of the continuumlevel contributions is normalized to an assumed energy-angle integrated continuum cross section which approximates the difference of Hopkin's measurement (Ref. 13) and the contribution from the first and second levels in ${ }^{6} \mathrm{LI}$. The steep rise of the pseudo-level cross sections from their thresholds and the use of fixed bin widths over finite angles produces anomalous structure in the individual cross sections which is especially apparent near the thresholds. Some effort has been made to smooth out these effects, but they remain to some extent.

MT=53. $\left(n, n_{1}\right) d$ Discrete Level Cross Sections

Cross section has p-wave penetrability energy dependence from threshold to $3.2 \mathrm{MeV}$. Matched at higher energies to a curve which 1ies 15-20\% above Hopkins (Ref. 13) and passes through the $10-\mathrm{MeV}$ point of Cookson ( $\operatorname{Re} f, 1.4$ ).

MT=57. $\quad\left(n, n_{2}\right) \gamma$ Cross Section

Rises rapidly from threshold, peaks at $5 \mathrm{mb}$ and falls off gradually to $20 \mathrm{MeV}$. No data available except upper limits. 
MT=102. $(n, \gamma)$ Cross Sections

Unchanged from Version IV, which was based on the thermal measurement of Jurney (Ref. 15) and the Pendlebury evaluation (Ref. 16) at higher energies.

$M T=103 . \quad(n, p)$ Cross Sections

Threshold to $9 \mathrm{MeV}$, based on the data of Ref. 17. Extended to 20 $\mathrm{MeV}$ through the 14-MeV data of Refs. 18 and 19.

$M T=105 . \quad(n, t)$ Cross Sections

Below $3 \mathrm{MeV}$, values are taken from the R-matrix analysis of Ref. 2 , which includes $(n, t)$ measurements from Refs. 20-24. Between 3 and $5 \mathrm{MeV}$, the values are based on Bartle's measurements (Ref. 24). At higher energies, the cross sections are taken from the evaluation of Ref. 16, extended to $20 \mathrm{MeV}$ considering the data of Kern (Ref. 25).

File 4. Neutron Secondary Angular Distributions

MT $=2$. Elastic Angular Distributions

Legendre coefficients determined as follows:

Below $2 \mathrm{MeV}$, coefficients up to $\mathrm{L}=2$ were taken from the R-matrix analysis of Ref. 2, which takes into account elastic angular distribution measurements from Refs. 9 and 10 above $2 \mathrm{MeV}$. The coefficients represent fits to the measurements of Refs. 13 and 26 in the 3.5-7.5 MeV range, that of Ref. 14 at $1 \mathrm{MeV}$, and those of Refs. 27-29 at $14 \mathrm{MeV}$. Extrapolation of the coefficients to $20 \mathrm{MeV}$ was aided by optical model calculations.

MT $=24 . \quad(n, 2 n)$ Angular Distributions

Laboratory distributions obtained by integrating over energy the 4-body phase-space spectra that result from transforming isotropic center-of-mass distributions to the laboratory system.

$M T=51-81 . \quad\left(n, n^{\prime}\right)$ Angular Distributions

Obtained by transforming distributions that are isotropic in the 3-body center-of-mass system to equivalent 2-body distributions in the laboratory system. $M T=53$ and 57 are treated as real levels and assumed to be isotropic in the two-body reference system. Data available indicate departure from isotropy for the first real level $(M T=53)$ and this anisotropy will be included in a later update. 
$M T=105 . \quad(n, t)$ Angular Distributions

Legendre coefficients obtained from the R-matrix analysis of Ref. 2 are supplied at energies below $1 \mathrm{MeV}$. The analysis takes into account $(n, t)$ angular distribution measurements from Refs. 23 and 30 .

File 5. Neutron Secondary Energy Distributions

$\mathrm{MT}=24 . \quad(n, 2 \mathrm{n})$ Energy Distributions

Laboratory distributions obtained by integrating over angle the 4body phase-space spectra that result from transforming isotropic center-of-mass distributions to the laboratory system.

File 8. Radioactive Nuclide Production

$\mathrm{MT}=103 . \quad(n, p){ }^{6} \mathrm{He}$

${ }^{6}$ He beta decays, with a half-1ife of $808 \mathrm{~ms}$, back to ${ }^{6} \mathrm{Li}$ with a probability of unity.

MT=105. $\quad(n, t){ }^{4} \mathrm{He}$

Tritium, which is the only radioactive product of this reaction, beta decays to ${ }^{3} \mathrm{He}$ with a probability of unity and with a lifetime of 12.33 years.

File 9. Radioactive Nuclide Multiplicities

MT=103. (n,p) Multiplicity

A multiplicity of one is ziven for the production of ${ }^{6} \mathrm{He}$.

$\mathrm{MT}=105 . \quad(n, t)$ Multiplicity

A multiplicity of one is given for the production of tritium.

File 12. Gamma-Ray Multiplicities

MT=57. $\left(n, n_{2}\right) \gamma$ Multiplicity

Multiplicity of one assumed for the 3.562-MeV gamma ray. Energy taken from reference 31 .

$M T=102 . \quad(n, \gamma)$ Multiplicity

Energies and transition arrays for radiative capture taken from Ref. 15, as reported in Ref. 31. The LP flag was used to describe the $\mathrm{MT}=102$ photons. 
File 14. Gamma-Ray Angular Distrábutions

MT=57. (n, n2) $\gamma$ Angular Distributions.

The gamma is assumed isotropic.

$\mathrm{MT}=102 . \quad(n, \gamma)$ Angular Distributions

The two high-energy gammas are assumed isotropic. Data on the 477-keV gamma indicate isotropy.

\section{File 33. Cross Section Covariances}

The relative covariances for $M T=1,2$, and 105 below $1 \mathrm{MeV}$ are given in File 33. They are based on calculations using the covariances of the R-matrix parameters in first-order error propogation.

MT $=1$. Total

Relative covariances are entered as NC-type sub-subsections, implying that they are to be constructed from those for $M T=2$ and 105. They are not intended for use at energies above $1.05 \mathrm{MeV}$.

$\mathrm{MT}=2$, 105. Elastic and $(n, t)$

Relative covariances among these two cross sections are entered explicitly as NI-type sub-subsections in the $L B=5$ (direct) representation. Although values for the $0.95-1.05 \mathrm{MeV}$ bin are repeated in a $1.05-20 \mathrm{MeV}$ bin, the covariances are not intended for use at energies above $1.05 \mathrm{MeV}$.

\section{REFERENCES}

1. G. M. Hale, L. Stewart, and P. G. Young, LA-6518-MS (1976).

2. G. M. Hale, Proc. Internat. Specialists Symposium on Neutron Standards and Applications, Gaithersburg (1977).

3. K. M. Diment and C. A. Uittley, AERF-PR/NP 15 and AERE-PR/NP 16 (1969). Also private communication to $L$. Stewart.

4. J. A. Harvey and N. W. Hill, Proc. Conf. on Nuclear Cross Sections and Technology, Vo1. 1, 244 (1975).

5. H. H. Knitter, C. Budtz-Jorgensen, M. Mailly, and R. Vogt, CBNM-VG (1976).

6. C. A. Goulding and P. Stoler, EANDC(US) $-176 \mathrm{U}, 161$ (1972).

7. D. G. Foster and D. W. Glasgow, Phys. Rev. C3, 576 (1971).

8. A. Bratenah1, J. M. Peterson, and J. P. Stoering, Phys. Rev. 110, 927 (1958), J. M. Peterson, A. Bratenah1, and J. P. Stoering, Phys. Rfv. 120, $521(1.960)$.

9. R. O. Lane, Ann. Phys. 12, 135 (1961).

10. H. H. Knitter and A. M. Coppola, FANDC(E)-57U (1967). Also Ref. 5 above.

11. D. S. Mather and L. F. Pain, AWRE-0-47/69 (1969).

12. V. J. Ashby et al., Phys. Rev. 129, 1771 (1963).

13. J. C. Hopkins, D. M. Drake, and H. Condé, Nucl. Phys. Al07, 139 (1968), and J. C. Hopkins, D. M. Drake, and H. Condé, LA-3765 (1967). 
14. J. A. Cookson and D. Dandy, Nucl. Phys. A91, 273 (1967).

15. E. T. Jurney, LASL, private communication (1973).

16. E. D. Pendlebury, AWRE-0-60/64 (1964).

17. R. Bass, C. Bindhardt, and K. Kruger, EANDC(E) $-57 \mathrm{U}$ (1965).

18. G. M. Frye, Phys. Rev. 93, 1086 (1954).

19. M. E. Battat and F. L. Ribe, Phys. Rev. 89, 8 (1953).

20. M. G. Sowerby, B. H. Patrick, C. A. Uttley, and K. M. Diment, J. Nucl. Energy 24, $323(1970) .{ }^{6} \mathrm{Li} /{ }^{10} \mathrm{~B}$ Ratio Converted Using ENDF/B-IV ${ }^{10} \mathrm{~B}(\mathrm{n}, \alpha)$ Cross Section.

21. G. P. Lamaze, 0. A. Wasson, R. A. Schrack, and A. D. Carlson, Proc. Internat. Conf. on the Interactions of Neutrons with Nuclei, Vol. 2,1341 (1976).

22. W. P. Poenitz, Z. Phys. 268, 359 (1974).

23. J. C. Overley, R. M. Sealock, and D. H. Ehlers, Nuc1. Phys. A221, 573 (1974).

24. C. M. Bartle, Proc. Conf. on Nuclear Cross Sections and Technology, Vo1. 2, 688 (1975), and private communication (1976).

25. R. D. Kern and W. E. Kreger, Phys. Rev. 112, 926 (1958).

26. R. Batchelor and J. H. Towle, Nucl. Phys. 47, 385 (1963).

27. A. H. Armstrong, J. Gamme1, L. Rosen, and G. M. Frye, Nuc1. Phys. 52, 505 (1964).

28. C. Wong, J. D. Anderson, and J. W. McC1ure, Nucl. Phys. 33, 680 (1962).

29. F. Merchez, N. V. Sen, V. Regis, and R. Bouchez, Compt. Rend. 260, 3922 (1965).

30. I. G. Schroder, E. D. McGarry, G. Deleeuw-Gierts, and S. Deleeuw, Proc. Conf. on Nuclear Cross Sections and Technology, Vol. 2, 240 (1975).

31. F. Ajzenberg-Selove, and T. Lauritsen, Nucl. Phys. A227, 55 (1974).

32. M. S. Coates et al., Neutron Standards Reference Data, IAEA, Vienna, p. 105 (1974).

33. S. J. Friesenhahn et al., INTEL-RT-7011-001 (1974).

34. W. Fort and J. P. Marquette, Proceedings of a Panel on Neutron Standard Reference Data, Nov. 20-24 (1972), IAEA, Vienna.

35. N. Jarmie et a1., BAPS 20, 596 (1975).

36. R. A. Hardekopf et al., LA-6188 (1977). 


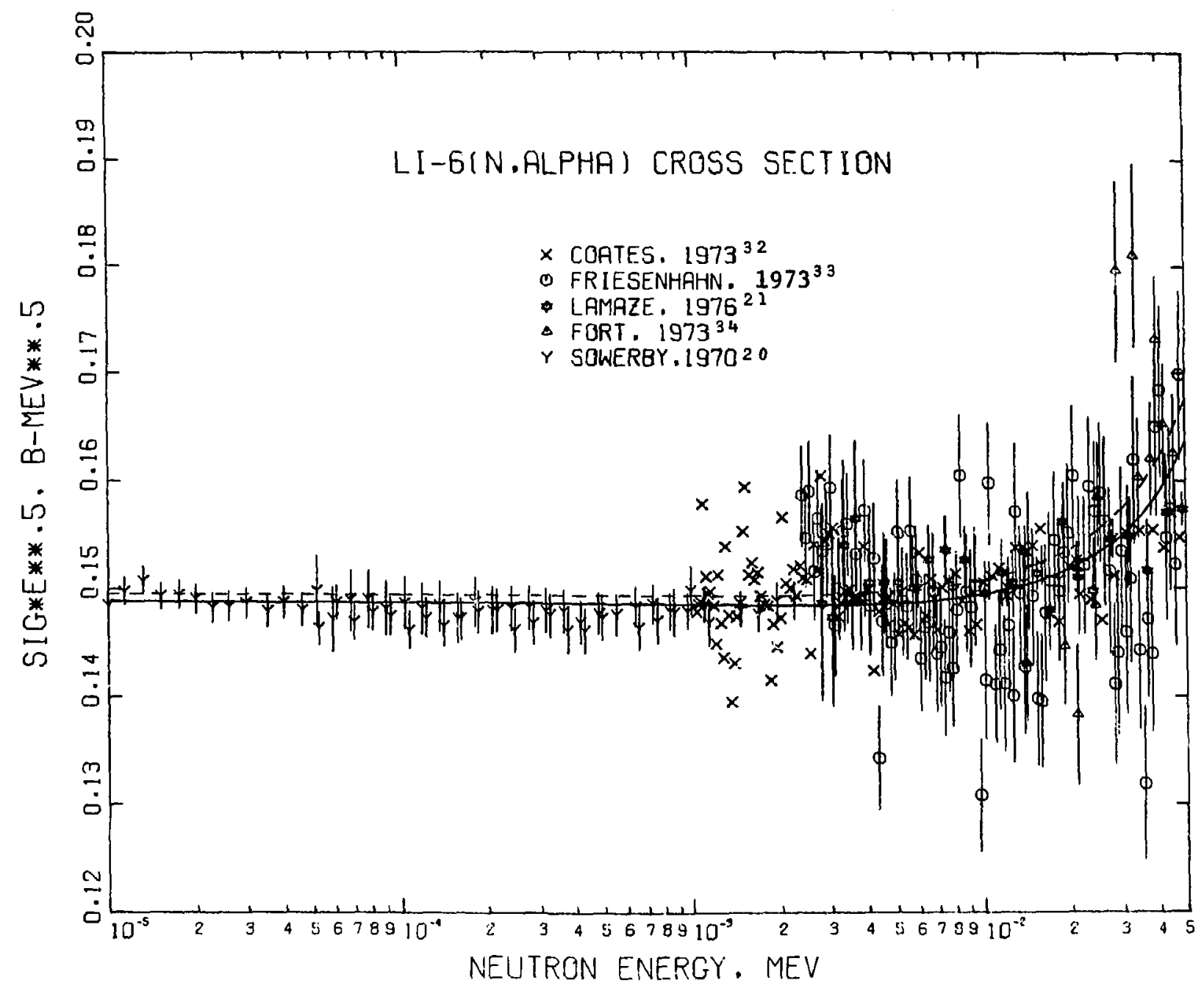

Fig. 1.

The Version $V{ }^{6} \mathrm{Li}(\mathrm{n}, \mathrm{t}){ }^{4} \mathrm{He}$ cross section times $\sqrt{\mathrm{E}_{\mathrm{n}}}$ plotted versus $E_{n}$ for neutron energies between $10 \mathrm{eV}$ and $50 \mathrm{keV}$. The dashed curve is ENDF/B-IV; the experimental data are from references $20,21,32-34$. 
文

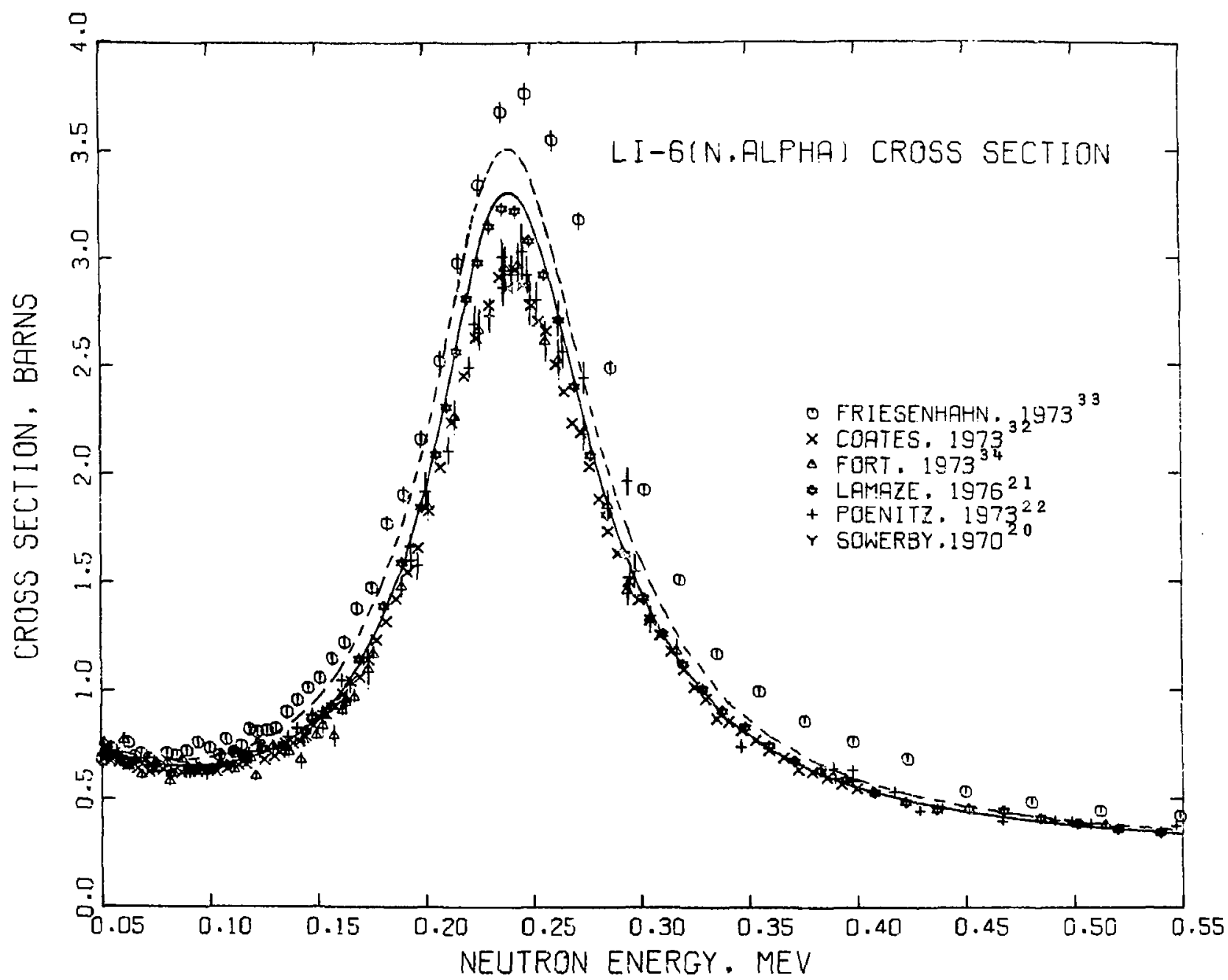

Fig. 2 .

The Version $\mathrm{V}^{6} \mathrm{Li}(\mathrm{n}, \mathrm{t})^{4} \mathrm{He}$ cross section from 50 to $550 \mathrm{keV}$. The dashed curve is ENDF/B-IV; the experimental data are from references 20-22, 32-34. 


\title{
SUMMARY DOCUMENTATION FOR ${ }^{7}$ Li
}

\author{
by \\ L. Stewart, D. G. Foster, Jr., M. E. Battat, and R. J. LaBauve \\ Los Alamos Scientific Laboratory \\ Los Alamos, New Mexico
}

\section{SUMMARY}

The ${ }^{7} \mathrm{Li}$ evaluation for ENDF/B-V (MAT 1272) was carried over from Version IV with only minor format modifications being included. The evaluation of all the neutron data except the total cross section is based upon a 1964 evaluation by Pendlebury (AWRE 0-61/64) as adapted by Battat and LaBauve for Version II of ENDF/B. The total cross section above $0.5 \mathrm{MeV}$ was re-evaluated by Foster as described below and photon production data added by LaBauve and Stewart for Version IV of ENDF/B. The evaluation covers the energy range from $10^{-5} \mathrm{eV}$ to $20 \mathrm{MeV}$. Covariance data are not included in the file but will be available in a new evaluation forthcoming from LASL.

\section{ENDF/B-V FILES}

File 1. General Information

MT=451. Descriptive data.

File 2. Resonance Parameters

$\mathrm{MT}=151$. Effective scattering radius $=0.28906 \times 10^{-12} \mathrm{~cm}$.

Resonance parameters not given.

File 3. Neutron Cross Sections

$M T=1$. Total Cross Section

Below $100 \mathrm{keV}$, based on analysis of available total cross section and radiative capture measurements (Hu58, Hu6O, Ho58, and Ho59) with an expression of the form $\sigma_{T}=\sigma_{n, n}+\sigma_{n, \gamma}$, where $\sigma_{n, n}=$ constant and $\sigma_{n, \gamma}$ has a $1 / V$ energy dependence. The analysis resulted in $\sigma_{\mathrm{n}, \mathrm{n}}=1.05 \mathrm{~b}$ and $\sigma_{\mathrm{n}, \gamma}=0.036 \mathrm{~b}$ at thermal.

100 - $500 \mathrm{keV}$, based on avallable experimental data (Hu58, Hy60, Ho58, and Ho59). 
$0.5-20 \mathrm{MeV}$, analysis by Foster (LASL) used. Evaluation from 0.5 to $1.3 \mathrm{MeV}$ based on measurements of Me70. Above $1.3 \mathrm{MeV}$, based on Go71, slightly normalized to improve agreelent with Fo71, $\mathrm{Br} 58$, Co52, and Pe60. Accuracy is approximately $2 \%$ at $0.5 \mathrm{MeV}$ and $1 \%$ above $1 \mathrm{MeV}$. Polynomial smoothing was used throughout.

MT=2. Elastic Cross Section

$0-100 \mathrm{keV}$, see MT=1 summary.

$0.1-20 \mathrm{MeV}$, based on experimental data of Ar63, Ba63B, Bo59, Gr59, La61, To56, Wi56, and Wo62.

MT=3. Nonelastic Cross Section (not included in file).

In order to determine the individual reaction cross sections, a total nonelastic cross section was evaluated with the elastic cross section, with each adjusted such that their sum equaled the MT=1 cross section. Experimental nonelastic measurements that were considered include Co59, Go59, Mc63, and Ri53.

MT=4. Inelastic Cross Section

Sum of $M T=51$ and 91 .

$\mathrm{MT}=16 . \quad(n, 2 n)$ Cross Section

Threshold - $20 \mathrm{MeV}$, smooth curve through experimental data of As63 at 10.2 and $14.1 \mathrm{MeV}$, smoothly extrapolated to $20 \mathrm{MeV}$, to obtain total $(n, 2 n)$. Then divided into $M T=16$ and $M T=24$ components as described under $\mathrm{MT}=24$.

$\mathrm{MT}=24 . \quad(\mathrm{n}, 2 \mathrm{nd}) \propto$ Cross Section

Threshold - $20 \mathrm{MeV}$, the total $(n, 2 n)$ cross section is divided into $(n, 2 n)^{6} \mathrm{Li}$ and $(n, 2 n d) \alpha$ components assuming that the latter reaction will dominate as the neutron energy increases above its threshold (9.98 MeV).

$\mathrm{MT}=51 . \quad\left(n, n^{\prime} \gamma\right)$ Cross Section

Threshold - $20 \mathrm{MeV}$, the energy-dependent cross section to the 478keV first-excited state of ${ }^{7} \mathrm{Li}$, which is the only ${ }^{7} \mathrm{Li}$ level stable to particle decay, is based mainly on the experimental data of $\mathrm{Ba} 63 \mathrm{~B}$, and Fr55 below $4 \mathrm{MeV}$ and Be62 near $14 \mathrm{MeV}$, with a smoath interpolation between and extrapolation to $20 \mathrm{MeV}$.

MT=91. $\quad\left(n, n^{\prime} t\right) \alpha$ Cross Section

Threshold - $20 \mathrm{MeV}$, the cross section to discrete and continuum states in ${ }^{7} \mathrm{Li}$ unstable to $t-\alpha$ breakup is based mainly on the experimental data of Ba63B, Ro62, and Th54, together with the results of Brown et al., and Osborne (see Hu58, Hu60). 
MT $=102$. (n, $\gamma)$ Cross Section

Below $150 \mathrm{keV}$, based on thermal value of $36 \mathrm{mb}$ (Hu60) with $1 / \mathrm{V}$ energy dependence until the cross section falls to $10 \mu \mathrm{b}$.

$0.15-20 \mathrm{MeV}$, held constant at $10 \mu \mathrm{b}$ to $15 \mathrm{MeV}$, decreasing to $8.6 \mu \mathrm{b}$ at $20 \mathrm{MeV}$.

MT=104. (n, d) Cross Section

Threshold - $20 \mathrm{MeV}$, smooth curve through the experimental data of $\mathrm{Ba53}, \mathrm{Ba} 63 \mathrm{~B}$, and Mi61.

$\mathrm{MT}=251$. Average Cosine of Scattering Angle

Derived from evaluated files.

MT $=252$. Average-Logarithmic Energy Decrement

Derived from evaluated files.

$\mathrm{MT}=253$. Gamma

Derived from evaluated files.

File 4. Neutron Angular Distributions

MT=2. Elastic Scattering Angular Distributions

Legendre coefficients given in center-of-mass system with transformation matrix (Al69). See Pe64 for evaluation details.

$M T=16$. $(n, 2 n)$ Angular Distributions

Tabular data (isotropic) in the laboratory system. See Pe64 for evaluation details.

$\mathrm{MT}=24 . \quad(\mathrm{n}, 2 \mathrm{nd}) \alpha$ Angular Distributions

Tabular data (isotropic) in the laboratory system. See Pe64 for evaluation details.

MT=51. ( $\left.n, n^{\prime} \gamma\right)$ Angular Distributions

Tabular data (isotropic) in the $\mathrm{cm}$ system. See Pe64 for evaluation details.

$\mathrm{MT}=91 . \quad\left(n, n^{\prime} t\right) \alpha$ Angular Distributions

Tabular data (anisotropic) in the laboratory system. See Pe64 for evaluatiun details. 
File 5. Neutron Energy Distributions

The tabulated spectra of Pendlebury (Pe64) were approximated by use of ENDF/B law 9, as described below.

$M T=16 . \quad(n, 2 n)$ Energy Distribution

Energy range is 8.3 to $20 \mathrm{MeV}$. Distribution approximated by ENDF/B law 9, with theta (MeV) equal to $0.21 \sqrt{E}$. This corresponds to an average theta of $0.7 \mathrm{MeV}$ in the 8.3 to $15 \mathrm{MeV}$ energy interval.

$\mathrm{MT}=24 . \quad(\mathrm{n}, 2 \mathrm{nd}) \alpha$ Energy Distribution

Energy range is 10 to $20 \mathrm{MeV}$. Distribution approximated by ENDF/B law 9, with theta (MeV) equal to $0.1133 \sqrt{E}$. This corresponds to an average theta of $0.4 \mathrm{MeV}$ in the 10 to $15 \mathrm{MeV}$ energy interval.

MT $=91 . \quad\left(n, n^{\prime} t\right) \alpha$ Energy Distribution

Distributions approximated using law 9. Theta values obtained by linear interpolation between following points:

$$
\begin{array}{lll}
E=2.821 & \mathrm{MeV} . & \text { Theta }=0.10 \mathrm{MeV} \\
\mathrm{E}=5.8 & \mathrm{MeV} . & \text { Theta }=0.70 \mathrm{MeV} \\
\mathrm{E}=8.0 & \mathrm{MeV} . & \text { Theta }=2.80 \mathrm{MeV} \\
\mathrm{E}=15.0 & \mathrm{MeV} . & \text { Theta }=5.35 \mathrm{MeV}
\end{array}
$$

Data include the cross section to the second level and do not always conserve energy.

File 12. Gamma-Ray Multiplicities

MT=51. $\quad\left(n, n^{\prime} \gamma\right)$ Multiplicity

The first level in ${ }^{7} \mathrm{Li}$ is the only known gamma emitter. Multiplicity of 1.0 assumed at all energies.

MT=102. (n, $\gamma)$ Multiplicity

Thermal capture spectrum measurements are inconclusive. Rough estimates of transition probabilities were made using level energies of Selove and Lauritsen (private communication). LP flag used to indicate primary transitions.

File 14. Gamma-Ray Angular Distributions

$M T=51 . \quad\left(n, n^{\prime} \gamma\right)$ Angular Distributions

Assumed isotropic at all energies. MT=102. $(n, y)$ Angular Distributions

Assumed isotropic at all energies. 


\section{REFERENCES}

Al69 H. Alter, Atomics International, private communication (1969).

Ar63 A. H. Armstrong and L. Rosen, WASH-1042, p. 23 (1963).

As63 v. J. Ashby, H. C. Catron, M. D. Goldberg, R. W. Hill, J. M. LeBlanc,

L. L. Newkirk, J. P. Stoering, C. J. Taylor, and M. A. Willianson, Phys . Rev. 129, 1771 (1963).

Ba53 M. F. Battat and F. L. Ribe, Phys. Rev. 89, 80 (1953).

Ba63A J. F. Barry, J. Nuc. Eng. 17, 273 (1963).

Ba63B R. Batchelor and J. H. Towle, Nuc. Phys. 47, 385 (1963).

Be62 J. Benveniste, A. C. Mitchel1, C. D. Schrader, and J. H. Zenger, Nuc1. Phys. 38, 300 (1962).

Bo59 N. A. Bostrom, I. L. Morgan, J. T. Prud"homme, P. L. Okhuysen, and O. M. Hudson, WADC-TN-59-107 (1959).

Br58 A. Bratenahl et a1., Phys. Rev. 110, 927 (1958).

Co52 J. H. Coon et al., Phys. Rev. 88, 562 (1952).

Co59 A. V. Cohen, Unpublished (1959).

Fo71 D. G. Foster, Jr., D. W. Glasgow, Phys. Rev. C3, 576 (1971).

Fr55 J. M. Freeman, A. M. Lane, and B. Rose, Phil. Mag. 46, 17 (1955).

Go59 V. M. Gorbachev and L. B. Poretskii, S. J. At. Eng. 4, 259 (1958), J. Nucl. Eng. 9 , 159 (1959).

Go71 C. A. Goulding et al., (RPI), private communication (1971).

Gr59 B. C. Groseclose, Mis. 59-4223 (1959).

Ho58 R. J. Howerton, UCRL 5226 (1958).

Ho59 R. J. Howerton, UCRL 5226 (Revised) (1959).

Hu58 D. J. Hughes and R. B. Schwartz, BNE 325, Second Edition (1958).

Hu60 D. J. Hughes, B. A. Magurno and M. K. Brussel, Supplement No. $I$ to BNL 325, Second Edition (1960).

La61 R. 0. Lane, A. S. Langsdorf, Jr., J. E. Monahan, and A. J. E1wyn, Annals of Physics 12, 135 (1961).

Mc63 M。H. McGregor, R. Booth, and W. P. Ball, Phys. Rev. 130, 1471 (1963).

Me70 J. W. Meadows and J. F. Whalen, Nucl. Sci. Eng. 41, 351 (1970).

Mi61 K. M. Mikhailina, A. A. Nomofilov, T. A. Romanova, V. A. Sviridov, F. A. Tikhomirov, and K. D. Tolstov, Sov. Progress in Neutron Physics, Ed. P. A. Krupchitskii (1961).

Pe60 J. M. Peterson et a1., Phys. Rev. 120, 521 (1960).

Pe64 E. D. Pendlebury, AWRE 0-61/64 (1964).

Ri53 F. L. Ribe, R. W. Davis, and J. M. Holt, LA-1589 (1953).

Ro62 L. Rosen and L. Stewart, Phys. Rev. 126, 1150 (1962).

Th54 R. G. Thomas, LA-1697 (1954).

To56 R. G. Thomas, M. Walt, R. B. Walton, and R. G. Allen, Phys. Rev. 101, 759 (1956).

Wi56 H. B. Willard, J. K. Bair, J. D. Kington, and H. O. Cohn, Phys. Rev. 101, 765 (i956).

Wo62 C. Wong, J. D. Anderson, and J. W. McClure, Nuc. Phys. 33, 680 (1962). 
SUMMARY DOCUMENTATION FOR ${ }^{10} 0_{B}$

\author{
by \\ G. M. Hale, L. Stewart, and P. G. Young \\ Los Alamos Scientific Laboratory \\ Los Alamos, New Mexico
}

\title{
I. SUMMARY
}

All cross sections below a neutron energy of $1.5 \mathrm{MeV}$ except the $(n, p)$ and $(n, t)$ reactions were revised for the Version $V$ evaluation of ${ }^{10} \mathrm{~B}$ (MAT 1305). The data above $1.5 \mathrm{MeV}$ were carried over from ENDF/B-IV. Other changes to the file include the addition of evaluated cross sections and secondary gamma-ray specira from the ${ }^{10} \mathrm{~B}(\mathrm{n}, \gamma)^{11} \mathrm{~B}$ reaction, as well as covariance data for cross sections below $1.5 \mathrm{MeV}$. Except for the covariance file, the evaluated data cover the energy range from $10^{-5} \mathrm{eV}$ to $20 \mathrm{MeV}$. Partial documentation is provided in LA-6472-PR (1976) and LA-6518-MS (1976).

\section{STANDARDS DATA}

The ${ }^{10} \mathrm{~B}(\mathrm{n}, \alpha)^{7} \mathrm{Li}$ and ${ }^{10} \mathrm{~B}\left(\mathrm{n}, \alpha_{1} \gamma\right)^{7} \mathrm{Li}$ reactions are neutron standards at energies below $100 \mathrm{keV}$. The major reactions below $1 \mathrm{MeV}$ were obtained for the Version $V$ evaluation from multichannel, multilevel $R$-matrix analyses of reactions in the ${ }^{11} B$ system, similar to those from which the Version IV evaluation were taken. New data have become available since Version IV was released and most of this new experimental information has been incorporated into the present analyses.

We have added Spencer's measurements of $\sigma_{T}(S p 73)$ and Sealock's ${ }^{10} B\left(n, \alpha_{1}\right)$ angular distributions (Se76) to the data set that was analyzed for Version IV. In addition, we have replaced Friesenhahn's integrated $\left(n, \alpha_{1}\right)$ cross section with the recent measurements of Schrack et al. (both with GeLi and NaI detectors) at NBS (Sc76), and have deleted Friesenhahn's total $(n, \alpha)$ cross section from the data set. The resulting fit to the $(n, \alpha)$ and $(n, \alpha \gamma)$ data is shown in Figs. 1 and 2 , respectively. The integrated ${ }^{10} \mathrm{~B}(\mathrm{n}, \alpha)$ cross section has changed neg1igibly from the Version IV results at energies below $200 \mathrm{keV}$. At higher energies, however, the $(n, \alpha)$ cross section has dropped significantly in response to the new NBS data. Unfortunately, the rest of the data in the analysis do not seem particularly sensitive to such changes in the $(n, \alpha)$ cross section, with the result that our calculated cross section must be considered quite uncertain at energies above $~ 300 \mathrm{keV}$. 


\section{ENDF/B-V FILES}

\section{File 1. General Information}

MT=451. Descriptive data.

\section{File 2. Resonance Parameters}

$\mathrm{MT}=451$. Effective scattering radius $=0.40238 \times 10^{-12} \mathrm{~cm}$.

Resonance parameters not included.

\section{File 3. Neutron Cross Sections}

The $2200 \mathrm{~m} / \mathrm{s}$ cross sections are as follows:

\begin{tabular}{|c|c|c|c|}
\hline$M T=1$ & Sigma & $=3839.1$ & \\
\hline $\mathrm{MT}=2$ & Sigma & 2.0344 & \\
\hline$M T=102$ & Sigma & 0.5 & \\
\hline $\mathrm{MT}=103$ & Sigma & 0.000566 & \\
\hline $\mathrm{MT}=107$ & Sigma & 3836.6 & \\
\hline $\mathrm{MT}=113$ & Sigma & 0.000566 & \\
\hline $\mathrm{MT}=700$ & Sigma & 0.000566 & \\
\hline $\mathrm{MT}=780$ & Sigma & 244.25 & \\
\hline $\mathrm{MT}=781$ & Sigma & 3592.3 & \\
\hline
\end{tabular}

MT $=1$. Total Cross Section

0 to $1 \mathrm{MeV}$, calculated from R-matrix parameters obtained by fitting simultaneously data from the reactions ${ }^{10} B(n, n),{ }^{10} B\left(n, \alpha_{0}\right)$, and ${ }^{10} B\left(n, \alpha_{1}\right)$. Total neutron cross-section measurements included in the fit are those of Bo52, Di67, and Sp73.

1 to $20 \mathrm{MeV}$, smooth curve through measurements of Di67, Bo52, Ts62, Fo61, Co52, and Co54, constrained to match R-matrix fit at $1 \mathrm{MeV}$.

$\mathbf{M T}=2$. Elastic Scattering Cross Section

0 to $1 \mathrm{MeV}$, calculated from the R-matrix parameters described for $M T=1$. Experimental elastic scattering data included in the fit are those of As70 and La71.

1 to $7 \mathrm{MeV}$, smooth curve through measurements of La71, Po70, and Ho69, constrained to be consistent with total and reaction cross section measurements.

7 to $14 \mathrm{MeV}$, smooth curve through measurements of Ho69, Co69, Te62, Va70, and Va65.

14 to $20 \mathrm{MeV}$, optical model extrapolation from $14-\mathrm{MeV}$ data.

$M T=4$. Inelastic Cross Section

Threshold to $20 \mathrm{MeV}$, sum of $\mathrm{MT}=51-85$. 
MT=51-61. Inelastic Cross Sections To Discrete States

$\begin{array}{rrrrrr}\mathrm{MT}=51 & \mathrm{Q}=-0.717 \mathrm{MeV} & \mathrm{MT}=55 & \mathrm{Q}=-4.774 \mathrm{MeV} & \mathrm{MT}=59 & \mathrm{Q}=-5.923 \mathrm{MeV} \\ 52 & -1.740 & 56 & -5.114 & 60 & -6.029 \\ 53 & -2.154 & 57 & -5.166 & 61 & -6.133 \\ 54 & -3.585 & 58 & -5.183 & & \end{array}$

Threshold to $20 \mathrm{MeV}$, based on $\left(n, n^{\prime}\right)$ measurements of Po70, Co69, Ho69, and Va70, and the $(n, x y)$ measurements of Da56, Da60, and Ne70 using a gamma-ray decay scheme deduced from La66, Al66, Se66A, and Se66B. Hauser-Feshbach calculations were used to estimate shapes and relative magnitudes where experimental data were lacking.

MT=62-85. Inelastic Cross Sections to Groups of Levels

These sections were used to group $\left(n, n^{\prime}\right)$ cross sections into 0.5MeV wide excitation energy bins between $E_{X}=6.5$ and $18.0 \mathrm{MeV}$. This representation was used in lieu of $\mathrm{MF}=5, \mathrm{MT}=91$ to more accurately represent kinematic effects.

Threshold to $20 \mathrm{MeV}$, integrated cross section obtained by subtracting the sum of MT $=2,51-61,103,104,107$, and 113 from $\mathrm{MT}=1$. Cross section distributed among the bands with an evaporation model using a nuclear temperature given by $\mathrm{T}=0.9728 \sqrt{\mathrm{E}_{\mathrm{n}}}$ (units $\mathrm{MeV}$ ), taken from Ir67.

$\mathrm{MT}=102 . \quad(n, \gamma)$ Cross Section

0 to $1 \mathrm{MeV}$, assumed $1 / \mathrm{V}$ dependence with thermal value of 0.5 barn.

1 to $20 \mathrm{MeV}$, assumed negligible, set equal to zero.

$M T=103 . \quad(n, p)$ Cross Section

Threshold to $20 \mathrm{MeV}$, sum of $\mathrm{MT}=700-703$.

$\mathrm{MT}=104 . \quad(\mathrm{n}, \mathrm{d})$ Cross Section

Threshold to $20 \mathrm{MeV}$, based on ${ }^{9} \mathrm{Be}(\mathrm{d}, \mathrm{n}){ }^{10} \mathrm{~B}$ measurements of $\mathrm{Si} 65$ and $B a 60$, and the $(n, d)$ measurement of Va65.

$M T=107 . \quad(n, \alpha)$ Cross Section

0 to $20 \mathrm{MeV}$, sum of $\mathrm{MT}=780$ and 781 .

$\mathrm{MT}=113 . \quad(\mathrm{n}, \mathrm{t} 2 \alpha)$ Cross Section

0 to $2.3 \mathrm{MeV}$, based on a single-level fit to the resonance measured at $2 \mathrm{MeV}$ by Da61, assuming $\mathrm{L}=0$ incoming neutrons and $\mathrm{L}=2$ outgoing tritons.

2.3 to $20 \mathrm{MeV}$, smooth curve through measurements of Fr56 and Wy58, following general shape of Da61 measurement from 4 to $9 \mathrm{MeV}$. 
$\mathrm{MT}=700-703$. (n,p) Cross Section to Discrete Levels

0 to $20 \mathrm{MeV}$, crudely estimated from the calculations of Po70 and the $(n, x y)$ measurements of Ne70. Cross section for MT=700 assumed identical to $\mathrm{MT}=113$ below $1 \mathrm{MeV}$. Gamma-ray decay scheme for ${ }^{10} \mathrm{~B}$ from La66.

$M T=780 . \quad\left(n, \alpha_{0}\right)$ Cross Section

0 to $1 \mathrm{MeV}$, calculated from the R-matrix parameters described for $M T=1$. Experimental $\left(n, \alpha_{0}\right)$ data input to the fit were those of Ma68 and Da61. In addition, the angular distributions of Va72 for the inverse reaction were included in the analysis.

1 to $20 \mathrm{MeV}$, based on Da61 measurements, with smooth extrapolation from 8 to $20 \mathrm{MeV}$. Da61 measurement above approximately $2 \mathrm{MeV}$ was renormalized by factor of 1.4 .

$\mathrm{MT}=781 . \quad\left(\mathrm{n}, \alpha_{1}\right)$ Cross Section

0 to $1 \mathrm{MeV}$, calculated from the R-matrix parameters described for $M T=1$. Experimental $\left(n, \alpha_{1}\right)$ data included in the fit are those of Sc76. In addition, the absolute differential cross-section measurements of Se76 were included in the analysis.

1 t. $20 \mathrm{MeV}$, smooth curve through measurements of Da61 and Ne70, with smooth extrapolation from 15 to $20 \mathrm{MeV}$. The Da61 data above approximately $2 \mathrm{MeV}$ were renormalized by a factor of 1.4 .

File 4. Neutron Angular Distributions

MT $=2$. Elastic Angular Distributions

0 to $1 \mathrm{MeV}$, calculated from the R-matrix parameters described for $M F=3, M T=1$. Experimental angular distributions input to the fit for both the elastic scattering cross section and polarization were obtained from the measurements of La71. Assignments for resonances above the neutron threshold are based on La71.

1 to $14 \mathrm{MeV}$, smoothed representation of Legendre coefficients derived from the measurements of La71, Ha73, Po70, Ho69, Co69, Ja69, and Va65, constrained to match the R-matrix calculations at $E_{\mathfrak{n}}=1$ $\mathrm{MeV}$.

14 to $20 \mathrm{Me}$ ?, optical model extrapolation of $14-\mathrm{MeV}$ data.

MT=51-85. Inelastic Angula:- Distributions

Threshold to $20 \mathrm{MeV}$, assumed isotropic in center-of-mass. 


\section{File 12. Gamma Ray Multiplicities}

MT=102. Capture Gamme, Rays

0 to $20 \mathrm{MeV}$, capture spectra and transition probabilities derived from the thermal data of Th67, after slight changes in the probabilities and renormalization to the energy levels of Aj75. The LP flag is used to conserve energy and to reduce significantly the amount of data required in the file. Except for the modification due to the LP flag, the thermal spectrum is used over the entire energy range.

MT=781. 0.4776-MeV Photon f rom the $\left(n, \alpha_{1}\right)$ Reaction

0 to $20 \mathrm{MeV}$, multiplicity of 1.0 at all energies.

File 13. Gamma-Ray Production Cross Sections

$M T=4 . \quad(n, n Y)$ Cross Sections

Threshold to $20 \mathrm{MeV}$, obtained from $M T=51-61$ using ${ }^{10} \mathrm{~B}$ decay scheme deduced from La66, Al66, Se66A, and Se66B.

$M T=103 . \quad(n, p r)$ Cross Sections

Threshold to $20 \mathrm{MeV}$, obtained from MT=701-703 using ${ }^{10} \mathrm{~B}$ decay scheme deduced from La66.

\section{File 14. Gamma Ray Angular Distributions}

$\mathrm{MT}=4$. $(\mathrm{n}, \mathrm{n} \gamma)$ Angular Distributions

Threshold to $20 \mathrm{MeV}$, assumed isotropic.

$\mathrm{MT}=102 . \quad(\mathrm{n}, \gamma)$ Angular Distributions

0 to $20 \mathrm{MeV}$, assumed isotropic.

$\mathrm{MT}=103 . \quad(\mathrm{n}, \mathrm{p} \gamma)$ Angular Distributions

Threshold to $20 \mathrm{MeV}$, assumed isotropic.

$\mathrm{MT}=781 . \quad\left(\mathrm{n}, \alpha_{1} \gamma\right)$ Angular Distribution

0 to $20 \mathrm{MeV}$, assumed isotropic.

\section{File 33. Cross-Section Covariances}

The relative covariances for the most important reactions open below $1 \mathrm{MeV}$ are given in File 33. These are calculated directly from the covariances of the R-matrix parameters, using first-order error propogation. 
$M T=2,780,781 .(n, n)\left(n, \alpha_{0}\right)$, and $\left(n, \alpha_{1}\right)$ Covariances.

0 to $1 \mathrm{MeV}$, relative covariances among these three reactions are entered explicitly using NI-type sub-stibsections in the LB=5 (direct) representation.

1 to $20 \mathrm{MeV}$, all covariances set equal to zero. Not intended for use in this energy range.

MT $=1,107$. Total and $(n, \alpha)$ Covariances.

0 to $1 \mathrm{MeV}$, for compactness, these covariances are constructed from those described above, using NC-type sub-subsections. The constructed covariances for the total cross section therefore neglect contributions from the $(n, \gamma),(n, p),(n, t)$, and $(n, n 1)$ reactions which are all presumed to be small in magnitude below $1 \mathrm{MeV}$. Note that although the total cross-section covariances are entered in the NC-type (derived) format, total cross-section data were included in the fit, and they influenced all the calculated covariances.

1 to $20 \mathrm{MeV}$, set equal to zero. Not intended for use in this energy range.

\section{REFERENCES}

Aj75 F. Ajzenberg-Selove, Nuc1. Phys. A248, 6 (1975).

A166 D. E. Alburger et a1., Phys. Rev. 143, 692 (1966).

As70 A. Asami and M. C. Moxon, J. Nucl. Energy 24, 85 (1970).

Ba60 R. Bardes and G. E. Owen, Phys, Rev. 120, 1369 (1960).

Be56 R. L. Becker and H. H. Barschall, Phys. Rev. 102, 1384 (1956).

Bo51 C. K. Bockelman et al., Phys. Rev. 84, 69 (1951).

Bo69 D. Bogart and L. L. Nichols, Nuc1. Phys. A125, 463 (1969).

Co52 J. H. Coon et a1., Phys. Rev. 88, 562 (1952).

Co54 C. F. Cook and T. W. Bonner, Phys. Rev. 94, 651 (1954).

Co67 S. A. Cox and F. R. Pontet, J. Nucl. Energy 21, 271 (1967).

Co69 J. A. Cookson and J. G. Locke, Nucl. Phys. A146, 417 (1970).

Co73 M. S. Coates et al., private communication to L. Stewart (1973).

Da56 R. B. Day, Phys. Rev. 102, 767 (1956).

Da60 R. B. Day and M. Walt, Phys. Rev. 117, 1330 (1960).

Da61 E. A. Davis et a1., Nucl. Phys. 27, 448 (1961).

Di67 K. M. Diment, AERE-R-5224 (1967).

Fo61 D. M. Fossan et al., Phys. Rev. 123, 209 (1961).

Fr56 G. M. Frye and J. H. Gammel, Phys. Rev. 103, 328 (1956).

Ha73 S. L. Hausladen, Thesis, Ohio Univ. Co0-1717-5 (1973).

Ho69 J. C. Hopkins, private communication to LASL (1969).

Ir67 D. C. Irving, ORNL-TM-1872 (1967).

La66 T. Lauritsen and F. Ajzenberg-Selove, Nucl. Phys. 78, 1 (1966).

La77 R. 0. Lane et a1., Phys. Rev. C4, 380 (1971).

Ma68 R. L. Macklin and J. H. Gibbons, Phys. Rev. 165, 1147 (1968).

Mo66 F. P. Mooring et al., Nucl. Phys. 82, 16 (1966).

Ne54 N. G. Nereson, LA-1655 (1954). 
Ne70 D. O. Ne11is et al., Phys. Rev. C1, 847 (1970).

Po70 D. Porter et al., AWRE $\underline{0}, 45 / 70$ (1970).

Sc76 R. A. Schrack et al., Proc. ICINN (ERDA-CONF-760715-P2), 1345 (1976).

Se76 R. M. Sealock and J. C. Overiey, Phys. Rev. C13, 2149 (1976).

Se66A R. E. Segel and R. H. Siemssen, Phys. Lett. 20,295 (1966).

Se66B R. E. Segel et al., Phys. Rev. 145, 736 (1966).

Si65 R. H. Siemssen et al., Nuc1. Phys. 69, 209 (1965).

Sp73 R. R. Spencer et al., EANDC(E) 147, AL (1973).

Te62 K. Tesch, Nucl. Phys. 37, $412(\overline{1962})$.

Th67 G. E. Thomas et al., Nucl. Instr. Meth. 56, 325 (1967).

Ts63 K. Tsukada and 0. Tanaka, J. Phys. Soc. Japan 18, 610 (1963).

Va63 V. Valkovic et al., Phys. Rev. 139, 331 (1965).

Va70 B. Vaucher et al., Helv. Phys. Acta, 43, 237 (1970).

Va72 L. Van der Zwan and K. W. Geiger, Nucl. Phys, A180, 615 (1972).

Wi55 H. B. Willard et al., Phys. Rev. 98, 669 (1955).

Wy 58 M. E. Wyman et al., Phys. Rev. 112, 1264 (1958). 


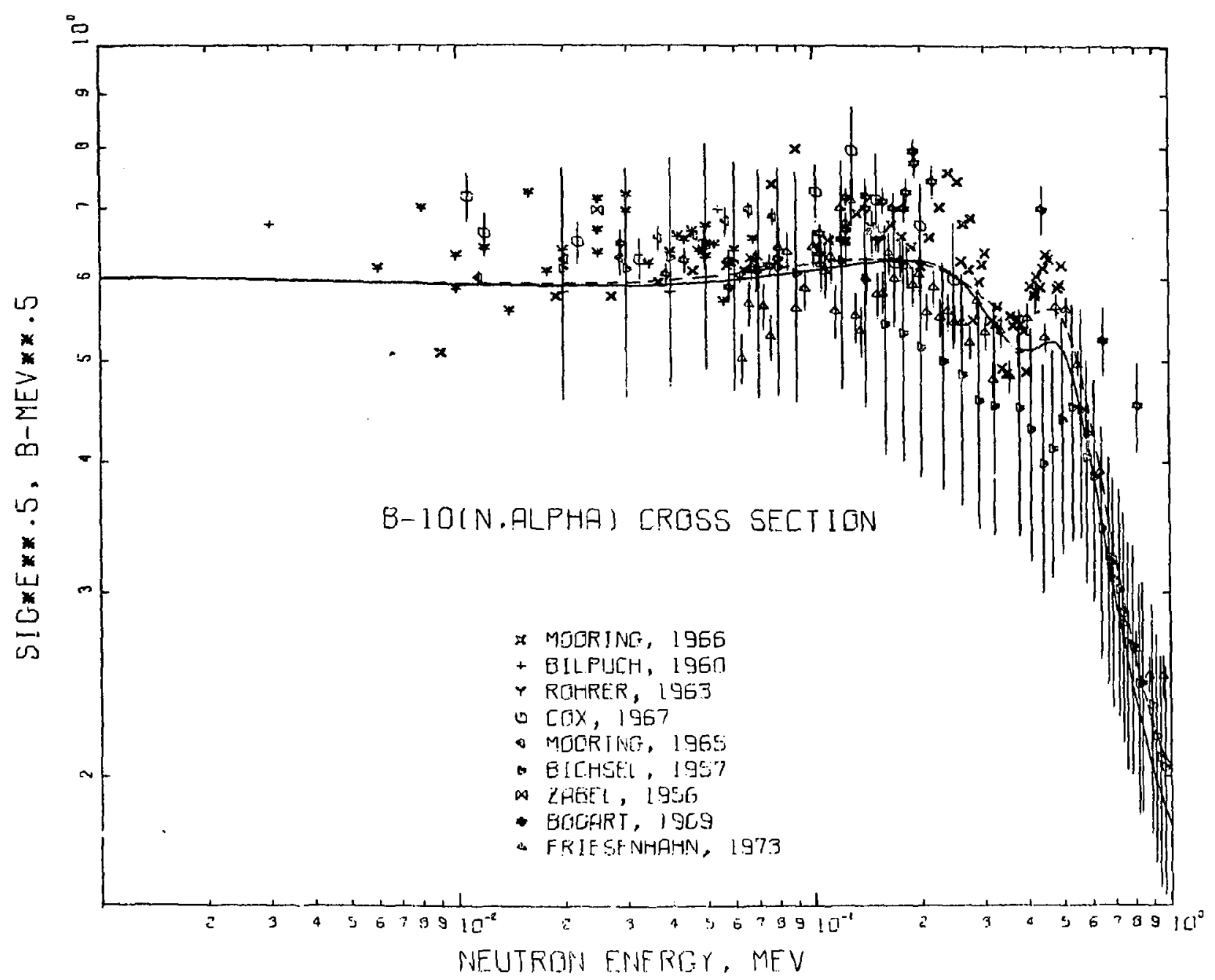

Fig. 1

Experimental and evaluated data for the ${ }^{1} 0_{B}(n, \alpha){ }^{7} \mathrm{Li}$ reaction from $1 \mathrm{keV}$ to $1 \mathrm{MeV}$. The solid curve is ENDF/B-V and the dashed curve is ENDF/B-IV. References for experimental data are given in LA-6518-MS. 


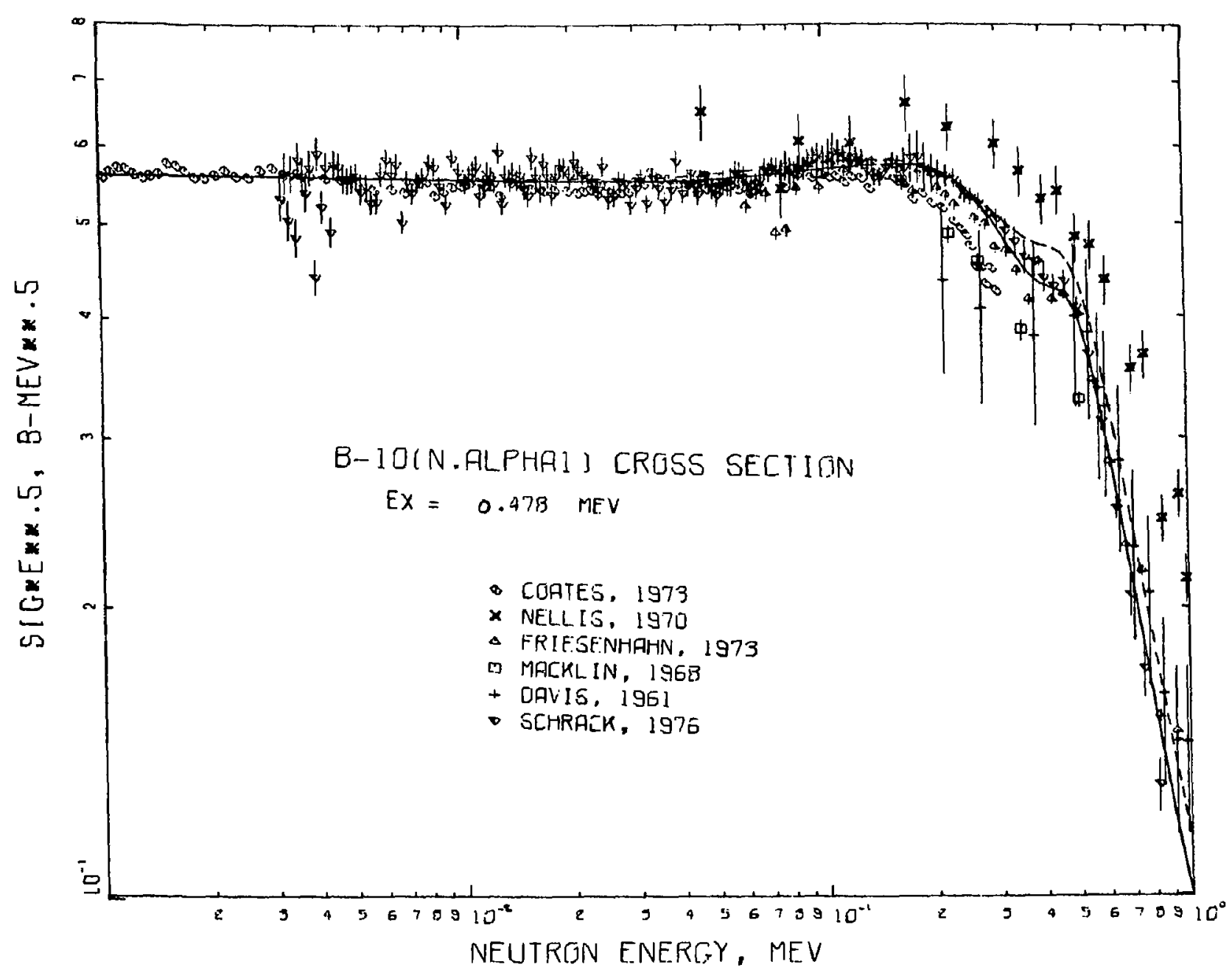

Fig. 2 .

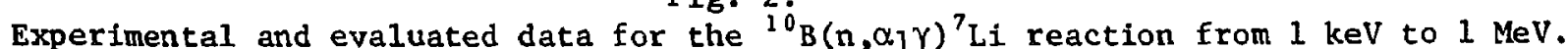
The solid. curve is ENDF/B-V and the dashed curve is ENDF/B-IV. References for experimental data are $\mathrm{Co73}, \mathrm{Da61}, \mathrm{Ma68}, \mathrm{Ne70}, \mathrm{Sc76}$, and those included in LA-6518-MS. 


\title{
SUMMARY DOCUMENTATION FOR ${ }^{14} \mathrm{~N}$
}

\author{
by \\ P. G. Young and D, G. Foster, Jr. \\ Los Alamos Scientific Laboratory \\ Los Alamos, New Mexico
}

\section{SUMMARY}

The ${ }^{14} \mathrm{~N}$ evaluation for ENDF/B-V (MAT 1275) is the same as Version IV except for updating of the covariance data format. Basic documentation for the evaluation is given in LA-4725 (1972), and revisions are described in LA-5375-PR (1973). The evaluation covers the energy range $10^{-5} \mathrm{eV}$ to $20 \mathrm{MeV}$.

\section{ENDF/B-V FILES}

File 1. General Information

MT=451. Descriptive Data

Thinning Note: Cross section data in $M F=3$ and 13 for $M T=4,16$, $51-82,102-108,700-704,720-723,740-741$, and 780-790 were thinned using a $2.5 \%$ thinning criterion. Similarly, in $M F=4$ the $M T=2,51-62$ angular distributions were thinned using the requirement that the interpolated angular distribution have an RMS average deviation from a fine-grid set of less than $2.5 \%$ and that the maximum excursion at any angle be less than $5 \%$.

File 2. Resonance Parameters

$\mathrm{MT}=151$. Effective Scattering Radius $=0.89014 \times 10^{-12} \mathrm{~cm}$.

Resonance parameters not given.

F1le 3. Neutron Cross Sections

The $2200 \mathrm{~m} / \mathrm{s}$ cross sections are as follows:

$\begin{array}{ll}\text { MT=1 } & \text { Sigma }=11.851 \text { barns } \\ \text { MT=2 } & \text { Sigma }=9.957 \text { barns } \\ \text { MT=3 } & \text { Sigma }=1.894 \text { barns } \\ \text { MT= 102 } & \text { Sigma }=0.075 \text { barns } \\ \text { MT= 103 } & \text { Sigma }=1.819 \text { barns }\end{array}$




\section{MT=1. Total Cross Section}

Zero to $1 \mathrm{eV}, \sigma_{\mathrm{T}}=9.957+0.3013 / \sqrt{\mathrm{E}}(\mathrm{b})$, from absorption of 1.894 $\mathrm{b}$ at $2200 \mathrm{~m} / \mathrm{s}$ and Me49 data above $1 \mathrm{eV}$.

$1 \mathrm{eV}$ to $10 \mathrm{keV}$, from data of Me49.

$10 \mathrm{keV}$ to $0.5 \mathrm{MeV}$, from Hi52, Bi59, Hu6l, and Bi62 with energy scales adjusted to match, normalized separately to join timeof-flight data smocthly at $0.5 \mathrm{MeV}$.

$0.5 \mathrm{MeV}$ to $20 \mathrm{MeV}$, from $\mathrm{Ca} 70$, He70, and Fo71 using Ca70 alone at sharp resonances.

Smoothed by appropriate fits. Log-log interpolation is good to $1.3 \%$ to $0.4 \mathrm{MeV}$. Linear interpolation is good to $0.5 \%$ from 0.4 to $20 \mathrm{MeV}$. Absolute error less than $1 \%$ above $0.75 \mathrm{MeV}$, but may rise to $5 \%$ in eV region.

\section{$\mathrm{MT}=2$. Elastic Scattering Cross Section}

Zero to $10 \mathrm{MeV}$, subtracted evaluated non-elastic cross section from the evaluated total, although direct elastic measurements of Fo55, Fo66, Bo57, Ch61, Ba67, Ph61, and Ne70 were considered.

10 to $12 \mathrm{MeV}$, Tre* Lon region.

12 to $20 \mathrm{MeV}$, Based upon data of Ch61, Ba67, Ne70, Ba63, and Bo68. MT=4. Total Inelastic Cross Section

Sum of $M T=51-82$ cross sections

Mi' $=16 . \quad(n, 2 n)$ Cross Section

Based upon data of Fe60, Br61, Bo65, and Pr60, estimated \pm 30 per cent error.

MT=51-62. Inelastic Cross Section to Discrete States

$$
\begin{array}{rrrrrr}
\mathrm{MT}=51 \mathrm{Q}=-2.313 \mathrm{MeV} & \mathrm{MT}=55 \mathrm{Q}=-5.691 \mathrm{MeV} & \mathrm{MT}=59 \mathrm{Q}=-7.028 \mathrm{MeV} \\
52 & -3.945 & 56 & -5.834 & 60 & -7.966 \\
53 & -4.913 & 57 & -6.198 & 61 & -8.061 \\
54 & -5.106 & 58 & -6.444 & 62 & -8.489
\end{array}
$$

Threshold to $15 \mathrm{MeV}$, from $(\mathrm{n}, \mathrm{n} \gamma)$ data of Di69, Or69, $\mathrm{C} 169, \mathrm{Bu} 69$, Ny69, Co68, and extensive measurements of Di73.

Above $15 \mathrm{MeV}$, smooth extrapolations.

MT=63-82 Inelastic Cross Section to Groups of Discrete States

Cross section is grouped into excitation energy bins of $0.5 \mathrm{MeV}$ width centered above Q-values between $-9.25 \mathrm{MeV}$ and $-18.75 \mathrm{MeV}$. 
The integrated cross section over these bands was adjusted such that the difference between tht total and non-elastic cross sections agreed with elastic data (see $M T=2$ ). The division of the cross section among the bands is based on Hauser-Feshbach and nuclear temperature calculations. Note that $(n, n p)$ and $(n, n \alpha)$ reactions are included with the discrete and binned inelastic data through use of LR flags ( $L R=28$ and 22 , respectively).

MI=102. Radiative Capture

Zero to $0.25 \mathrm{MeV}, 1 / \mathrm{V}$ from $75 \mathrm{mb}$ ( $\pm 10 \%$ ) at thermal (Ju63).

0.25 to $1 \mathrm{MeV}$. Transition region.

1 to $20 \mathrm{MeV}$. Deduced from ${ }^{14} \mathrm{~N}(\mathrm{p}, \mathrm{\gamma})^{15} \mathrm{O}$ data of $\mathrm{Ku} 70$, assuming charge independence. Energy scale adjusted to match foot hills of $(p, \gamma)$ giant resonance to resonance clusters observed in ${ }^{15} \mathrm{~N}$ compound nucleus.

MT=103. (n,p) Cross Section

Sum of $M T=700-704$.

MT $=104 . \quad(n, d)$ Cross Section

Sum of $M T=720-723$.

MT=105. (n,t) Cross Section

Sum of $M T=740-741$.

$\mathrm{MT}=107 . \quad(n, \alpha)$ Cross Section

Sum of MT $=780-790$.

MT $=108 . \quad(n, 2 \alpha)$ Cross Section

Based on L152, Mo67, and Hauser-Feshbach calculation.

MT=700. ( $\pi, p)$ Cross Section to ${ }^{14} \mathrm{C}$ Ground State

Zero to $4 \mathrm{MeV}$, from data of Jo50 and Ga59.

4 to $13 \mathrm{MeV}$, from inverse reaction data of Wo67.

13 to $20 \mathrm{MeV}$, smooth extrapolation.

$M T=701-704,(n, p)$ Cross Sections to ${ }^{14} \mathrm{C}$ Excited States

$\mathrm{MI}=701 \mathrm{Q}=-5.468 \mathrm{MeV} \quad \mathrm{EII}=6.095 \mathrm{MeV}(6.58,6.89-\mathrm{MeV}$ level excitation cross sections are also included)

$\mathrm{MT}=702 \quad Q=-6.102 \mathrm{MeV} \quad \mathrm{Ex}=6.728 \mathrm{MeV}$ 
$\mathrm{MT}=703 \quad \mathrm{Q}=-6.385 \quad \mathrm{Ex}=7.012 \mathrm{MeV}$

$704 \quad-6.711 \quad 7.337$

Threshold to $15 \mathrm{MeV}$, from (n,pY) data of 0r69, Di69, Cl69, Bu69, and Ny69.

15 to $20 \mathrm{MeV}$, smooth extrapolations.

MT=720. (n,d) Cross Section to ${ }^{13} \mathrm{C}$ Ground State.

Threshold to $15 \mathrm{MeV}$, from inverse cross section data of Ch61, Be63 near threshold and direct data of Mi68, Fe67, Ca57, and Za63 at $14 \mathrm{MeV}$.

15 to $20 \mathrm{MeV}$, smooth extrapolations.

$\mathrm{MT}=721-723(\mathrm{n}, \mathrm{d})$ Cross Section to Excited ${ }^{13} \mathrm{C}$ Levels

$$
\begin{array}{rcc}
\mathrm{MT}=721 & \mathrm{Q}=-8.411 \mathrm{MeV} & \mathrm{Ex}=3.086 \mathrm{MeV} \\
722 & -9.009 & 3.684 \\
723 & -9.179 & 3.854
\end{array}
$$

Threshold to $15 \mathrm{MeV}$, direct data of Fe67, Za63, Ca57 and (n,dy) data of Or69 and Di69 below $13 \mathrm{MeV}$.

15 to $20 \mathrm{MeV}$, smooth extrapolations.

MT=740-741. (n,t) Cross Sections to ${ }^{12} \mathrm{C}$ Ground and $4.439 \mathrm{MeV}$-Excited State.

Threshold to $15 \mathrm{MeV}$, direct data of Ga59, Sc66, Re67, and Fe67.

15 to $20 \mathrm{MeV}$, smooth extrapolations.

MT=780-790. $(n, \alpha)$ Cross Section to Discrete ${ }^{11} B$ States

$\begin{array}{ccc}\mathrm{MT}=780 & \mathrm{Q}=-0.157 \mathrm{MeV} & \mathrm{Ex}=0.000 \mathrm{MeV} \\ 781 & -2.282 & 2.124 \\ 782 & -4.602 & 4.444 \\ 783 & -5.176 & 5.019 \\ 784 & -6.900 & 6.743 \\ 785 & -6.950 & 6.793 \\ 786 & -7.453 & 7.296 \\ 787 & -8.153 & 7.996 \\ 788 & -8.723 & 8.566 \\ 789 & -9.082 & 8.925 \\ 790 & -9.342 & 9.185\end{array}$

Threshold to $6 \mathrm{MeV}$, from direct data of Jo50, Ge59, and Sc66.

6 to $15 \mathrm{MeV}$, used above direct data, together with $(\mathrm{n}, \mathrm{\alpha r})$ data of Ha59, Di69, Or69, Ny69, anó Bu69. Near $14 \mathrm{MeV}$, direct $(n, \alpha)$ data of Li52, Ba68, Le68, and Ma68 were also used. 
15 to $20 \mathrm{MeV}$, smooth extrapolations.

File 4. Secondary Neutron Angular Distributions

MT=2. Elastic Angular Distributions

Zero to $8 \mathrm{MeV}$, based upon resonance theory analysis of data of Fo55, Fo66, Bo57, Ch61, and Ba67, using parameters from total and partial cross section analyses and Aj70.

8 to $15 \mathrm{MeV}$, based upon data of $\mathrm{Ch} 61, \mathrm{Ba67}, \mathrm{Ne} 70$, and Ba63.

15 to $20 \mathrm{MeV}$, based upon optical model calculation.

$M T=16$. Angular Distribution for $(n, 2 n)$ Reaction

In the absence of data, isotropy in the $\mathrm{cm}$ system is assumed, and the corresponding 3-body phase-space transformed to the laboratory system is given. For any reasonable $\mathrm{cm}$ distribution, the strong forward peaking of the transformation will dominate. Normalized for trapezoidal integration.

$M T=51$ to 62 . Angular Distributions for Ine:astic Scattering

Above $7 \mathrm{MeV}$ taken from proton data of Do64, Ha70, and 0d60 assuming charge symmetry, and neutron data of Ba63. Threshold shapes modeled after Hauser-Feshbach calculations.

MT=63-82. Angular Distributions for Inelastic Scattering

Assumed isotropic in $\mathrm{cm}$ at all energies.

File 5. Energy Distribution of Secondary Neutrons

$M T=16$. Spectrum of $(n, 2 n)$ Secondary Neutrons

In the absence of data, only the 3-body phase-space distribution is given. Normalized for trapezoidal integration.

File 12. Photon Multiplicities

$M T^{\prime}=102 . \quad(n, \gamma)$ Multiplicities

Zero to $0.25 \mathrm{MeV}$, thermal spectrum based primarily upon measurements of Th67, Jo69, Gr68, and Mo62. LP flags were used to designate primary gamma rays.

0.25 to $1 \mathrm{MeV}$. Transition region where thermal spectrum is phased into single ground-state transition.

1 to $20 \mathrm{MeV}$, deduced from ${ }^{14} \mathrm{~N}(\mathrm{p}, \gamma)^{15} \mathrm{O}$ data of $\mathrm{Ku} 70$, who observed no significant transitions except to ground state. 


\section{File 13. Photon-Production Cross Sections}

All $(n, \gamma)$ cross sections agree with the excitation cross sections in $M F=3$ via the relevant decay scheme $(A j 68, A j 70)$. However, $M T=104,105$ include contributions from ( $n, n p \gamma)$ and ( $n, n d y)$.

$M T=4 . \quad(n, n \gamma)$ Cross Section

From data of Ha59, Di69, Or69, Cl69, Bu69, Ny69, Co68, and Di73.

MT $=103 . \quad(n, p \gamma)$ Cross Section

From data of Di69, Or69, Bu69, Ny69, and C269.

$M T=104 . \quad(n, d \gamma)+\left(n, n_{p} \gamma\right)$ Cross Section

From data of Di69, Or69, Bu69, Ny69, and Cl69.

$\mathrm{MT}=105 . \quad(\mathrm{n}, \mathrm{t} \gamma)+(\mathrm{n}, \mathrm{nd} \gamma)$ Cross Section

$(n, t \gamma)$ estimated from $(n, t)$ as discussed under $M F=3$ MT $=741$. $(n, d n \gamma)$ estimated from MT=63-82.

$\mathrm{MT}=107 . \quad(n, \infty \gamma)$ Cross Section

From $(n, \alpha)$ data of Ga59, Sc66 and $(n, 0 \gamma)$ data of Ha59, Di69, Ny69, Or69, and Bu69.

File 14. Photon Angular Distributions

Data on 9 strongest lines from inelastic scattering and particle reactions taken from Mo64.

$\mathrm{MT}=4$. Inelastic Scattering to ${ }^{14} \mathrm{~N}$

The 1.63- and 4.91-MeV gamma are given as anisotropic. All remaining gamma rays are isotropic.

$\mathrm{MT}=102 . \quad(\mathrm{n}, \gamma)$ Angular Distributions

Zero to $0.4 \mathrm{MeV}$, all photons are isotropic.

0.4 to $20 \mathrm{MeV}$, anisotropic distribution for the single ground state transition is based upon ${ }^{14} \mathrm{~N}\left(\mathrm{p}, \gamma_{\mathrm{O}}\right)^{15} \mathrm{O}$ data by Ku70.

$\mathrm{N}_{\mathrm{I}} \mathrm{T}=103$. ( $\left.\mathrm{n}, \mathrm{p} \gamma\right)$ Angular Distributions

All isotropic.

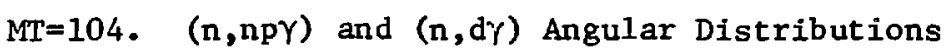

All isotropic except $3.85-\mathrm{MeV}$ gamma ray. 
$\mathrm{MT}=105 . \quad(n, n d \gamma)$ and $(n, t \gamma)$ Angular Distributions

All isotropic.

$M T=107 . \quad(n, \alpha \gamma)$ Angular Distributions

All isotropic.

File 33. Neutron Cross Section Corvariances

$\mathrm{MT}=1,2,4,102,103$, and 107. Smooth Cross Section Covariances

Covariances are based upon estimates of the uncertainty in the experimental measurements and theoretical calculations used in in the evaluation. Format updated for Version V. Derived covariances are included explicitly.

\section{REFERENCES}

Aj68 F.Ajzenberg-Selove, and T. Lauritsen, (HAV), Nuc1. Phys. A114, 1 (1968). Aj70 F.Ajzenberg-Selove (PEN), Nuc1. Phys. A152, 1 (1970).

Ba63 R. W. Bauer et al., (LRL), Nucl. Phys. 47, 241 (1963).

Ba67 R. W. Bauer et al., (LRL), Nucl. Phys. A93, 673 (1967).

Ba68 R. Bachinger and M. Uh1, (IRK), Nucl. Phys. A116 673 (1968).

Be63 R. E. Benenson and R. Yaramis, (COL), Phys. Rev. 129, 720 (1963).

Bi59 E. G. Bilpuch et al., (DKE), Private communication to BNL (1959),

B162 E. G. B1lpuch et al., (DKE), Private communication to R. J. Howerton (i962).

Bo57 N. A. Bostrom et al., (TNC), WADC-TR-57-446 (1957).

Bo65 M. Bormann et a1., (HAM), Nucl. Phys. 63, 438 (1965).

Bo68 F. Boreli et al., (TEX), Phys. Rev. 174, 1221 (1968).

Br61 0. D. Brill et al., (RUS), Sov. Phys. Dok1. 6 , 24 (1961).

Bu69 P. S. Buchanan (TNC). Private communication (1969).

Ca57 R. R. Carlson (LAS), Phys. Rev. 107, 1094 (1957).

Ca70 A. D. Carlson and R. J. Cerbone, (GGA), Nucl. Sci. Eng. 42, 28 (1970).

Ch61 L. F. Chase et al., (LOK), AFSWC-TR-61-15 (1961).

Gl69 G. Glayeux and G. Grenier (FR), CEA-R-3807 (1969).

Co68 H. Condé et al., (SWD), Neut. Cross Sect. and Tech. Conf., Washington, D.C., p. 763 (1968).

Di69 J. K. Dickens and F. G. Perey, (GRL), Nuc. Sci. Eng. 36, 280 (1969).

D170 J. K. Dickens et al., ORNL-4864 (1973).

Do64 D. F. Donovan et a1., (BNL), Phys. Rev. 133, B113 (1964).

Fe60 J. M. Ferguson and W. E. Thompson, (NRD), Phys. Rev. 118, 228 (1960).

Fe67 P. Fessenden and D. R. Maxson, (BRN), Phys. Rev, 158, 948 (1967).

Fo55 J. I. Fowler and C. H. Johnson, (GRL), Phys. Rev. $\frac{98}{9}, 728$ (1955).

Fo66 J. L. Fowler et al., (ORL), Neut. Cross Sect, and Tech. Conf., Wash. D.C., p. 653 (1968).

Fo71 D. G. Foster, Jr, and W. Glasgow, (PNL), Phys. Rev. C3, 576 (1971).

Ga59 F. Gabbard et al,, (RIC), Nucl. Phys. 14, 277 (1959).

Gr68 R. C. Greenwood, (MTR), m Phys. Lett. $2 \overrightarrow{7 \mathrm{~B}}, 274$ (1968).

Ha59 H. E. Hall and T. W. Bonner, (RIC), NucI. Phys. 14, 295 (1959).

Ha70 L. Hansen, (IRL), Private communication (1970).

He70 H. T. Heaton et al., (NBS), Bull. Am. Phys. Soc. 15, 568 (1970). 
Hi52 J. J. Hinchey et al., (MIT), Phys. Rev. 86, 483 (1952).

Hu61 C. M. Huddleston and F. P. Mooring, (ANL), ANL-6376, p. 13 (1961).

Jo50 C. H. Johnson and H. H. Barschall, (WIS), Phys. Rev. 80, 818 (1950).

Jo69 L. Jonsson and R. Harde11, (SWD), Symposium on Neutron Capture Gamma Rays, Studsvik (1969).

Ju63 E. T. Jurney and H. T, Motz, (LAS), ANL-9797, p. 241 (1963).

Ku70 H. M Kuan et al., (STF), Nucl. Phys. A151, 129 (1970).

Le68 B. Leroux et al., (FR), Nucl. Phys. A116, 196 (1968).

Li52 A. B. Lillie, (RIC), Phys. Rev. 87, 716 (1952).

Ma68 D. P. Maxson et a1., (BRN), Nuc1. Phys, A110, 609 (1968).

Me49 E. Melkonian, (COL), Phys. Rev. 76, 1750 (1949).

Mi68 D, Miljanio et a1., (YUG), Nuc1. Phys. A106, 401 (1968).

Mo62 H. T. Motz et a1., (LAS), Pile Neutron Research in Physics (IAEA, Vienna 1962), p. 225

Mo64 I. L. Morgan et al., (TNC), TNC Nuc1. Phys. Div. Ann. Rpt. (Aug. 1964).

Mo67 J. Mosner et al., (GER), Nuc1. Phys. A103, 238 (1967).

Ne70 D. 0. Nellis, (TNC), Private communication (1970).

Ny69 K. Nyberg, (SWD), Private communication to L. Stewart (1969).

od60 Y. Oda et a1., (TOK), J. Phys. Soc. Japan 15, 760 (1960).

0r69 V. J. Orphan et al., (GGA), GA-8006 (1969).

Ph61 D. D. Phillips (LAS), Private communication to R. J. Howerton (1961).

Pr60 J. T. Prudhomme et al., (TNC), AFSWC-TR-60=30 (1960.

Re67 D. Rendic (YUG), Nucl. Phys. A91, 604 (1967).

Sc66 W. Scobel et al., (HAM), Z. Physik 197, 124 (1966).

Th67 G. F. Thomas et al., (ANL), Nucl. Instr. Meth. 56, 325 (1967).

Wo67 C. Wong et al., (LRL), Phys. Rev. 160, 769 (1967).

Za63 M. R. Zatzick and D. R. Maxson, (BRN), Phys. Rev. 129, 1728 (1963). 
SUMMARY DOCUMENTATION FOR ${ }^{15} \mathrm{~N}$

\author{
by \\ E. D. Arthur, P. G. Young, and G. M. Hale \\ Los Alamos Scientific Laboratory \\ Los Alamos, New Mexico
}

\title{
I. SUMMARY
}

The ${ }^{15} \mathrm{~N}$ for ENDF/B-V (MAT 1307) is a new evaluation performed originally in 1975 and updated in 1978 for Version $V$. The data set covers the energy range $10^{-5} \mathrm{eV}$ to $20 \mathrm{MeV}$ and is partially documented in LA-6123-PR (1975) and LA-6164-PR (1975).

\section{ENDF/B-V FILES}

\section{File 1. General Information}

MT $=451$. Descriptive Data

General approach: The lack of experimental data, with the exception of total cross section measurements, led us to obtain cross sections with a variety of nuclear model codes. We used an energy dependent R-matrix analysis to $f i t$ the total cross section data of Ze71 for neutron energies from 0.8 to $5.4 \mathrm{MeV}$. As a starting point we used the level scheme reported in Ze71. Also used in the R-matrix analysis were the elastic angular distributions of Si62 and Ze71 in the energy range from 1.9 to $3.64 \mathrm{MeV}$. The parameters of the R-matrix analysis were then used to generate cross sections and to determine Legendre coefficients in the range from $1.0 \mathrm{E}-05$ to $5.4 \mathrm{MeV}$. In the energy region from 6 to 20 $\mathrm{MeV}$ we used two statistical model codes to generate cross sections. With the first, COMNUC, we computed the angular distributions of elastically scattered neutrons. The Legendre coefficients obtained were then joined smoothly with those from the R-matrix analysis. We also obtained angular distributions of neutrons leading to the first seven excited states of ${ }^{15} \mathrm{~N}$. To generate neutron transmission coefficients for this part of the analysis, we used the global optical model parameters of Wilmore and Hodgson as reported by Pe74, and adjusted them to give good agreement with the measured total crass section. The generation of capture, inelastic, and non-elastic cross sections as well as neutron, gammaray, and charged-particle spectra was done with the statistical model code, GNASH. This newly developed code (Yo77) is based largely on the statistical model formalism of Uh71. Again, neutron 
transmission coefficients were based on the optical model parameters of Wilmore and Hodgson. Transmission coefficients were generated for protons, deuterons, and ${ }^{4} \mathrm{He}$, with the appropriate global forms reported in Pe74. For tritons we used the global parameters for ${ }^{3} \mathrm{He}$ reported in Pe74 since these were consistent with triton optical model parameters for individual light nuclei as compiled by Pe74. The Gilbert-Cameron level density model was used with standard values for level density parameters (Gi64). To account for direct and semi-direct processes we used the preequilibrium model of Blann and Cline (C8.71) and assumed a $10 \%$ preequilibrium fraction.

Eile 2. Resonance Parameters

$\mathrm{MT}=151$. Effective scattering radius $=0.592916 \times 10^{-12} \mathrm{~cm}$

Resonance parameters not given.

File 3. Neutron Cross Sections

The $2200 \mathrm{~m} / \mathrm{s}$ Cross Sections are:

$$
\begin{aligned}
\text { MT }=1 & \text { Sigma }=4.4185 \mathrm{~b} \\
M T=2 & \text { Sigma }=4.418 \mathrm{~b} \\
M T=102 & \text { Sigma }=24.0 \mathrm{E}-06 \mathrm{~b}
\end{aligned}
$$

MT=1. Total Cross Section

From $1.0 \mathrm{E}-05 \mathrm{eV}$ to $5.4 \mathrm{MeV}$ total cross section values are based on the R-matrix analysis described above. From 5.5 to $20 \mathrm{MeV}$, the evaluated cross section is based on the smoothed results of Ze71.

MT=2. Elastic Scattering Cross Section

Zero to $20 \mathrm{MeV}$, subtracted evaluated non-elastic irom total to give elastic cross section.

MT=4. Inelastic Cross Section

Sum of $M T=22,28,51-57$, and 91 cross sections.

$M T=16 . \quad(n, 2 n)$ Cross Section

Based on smooth curve drawn through results of the statistical model calculations described before.

$\mathrm{MT}=22 . \quad(n, n \alpha)$ Cross Section

Based on smoothed results of statistical model calculation.

$M T=28$. ( $n, n p)$ Cross Section

Based on smoothed results of statistical model calculation. 
$\mathrm{MT}=51-57$. Inelastic Cross Section to Discrete States

$\begin{array}{rcrc}M T=51 & Q=-5.27 \mathrm{MeV} & \text { MT=55 } & Q=-7.301 \mathrm{MeV} \\ 52 & -5.299 & 56 & -7.566 \\ 53 & -6.324 & 57 & -8.313 \\ 54 & -7.155 & & \end{array}$

MT=91. $\quad(n, n)$ To Continuum

Continuum defined to begin at $0.1 \mathrm{MeV}$ above last ${ }^{15} \mathrm{~N}$ discrete level included in calculation, 1.e., continuum begins at $8.413 \mathrm{MeV}$.

MT=102. $\quad(n, \gamma)$ Cross Section

Zero to $0.010 \mathrm{MeV}, 1 / \mathrm{V}$ from $24.0 \mathrm{E}-06 \mathrm{~b}$ at thermal

6 to $20 \mathrm{MeV}$ calculated from statistical model with Axel (Ax62) giant dipole resonance approximation for gamma-ray transmission coefficients.

0.010 to $6 \mathrm{MeV}$, cross section based on smoothed curve from region where $1 / \mathrm{V}$ dependence ends to region where calculated values begin.

$\mathrm{MT}=103 . \quad(n, p)$ Cross Section

Sum of MT=700, 701, and 718 cross sections.

$\mathrm{MT}=104 . \quad(\mathrm{n}, \mathrm{d})$ Cross Section

Sum of $M T=720,721,722$, and 738 cross sections.

$\mathrm{MT}=105$. $\quad(n, t)$ Cross Section

Sum of $M T=740,741$, and 758 cross sections.

MT=107. $\quad(n, \alpha)$ Cross Section

Sum of $M T=780-785$ and 798 cross sections.

$\mathrm{MT}=700,701$. (n,p) Cross Section to ${ }^{15} \mathrm{C}$ Ground and First Excited States

$\mathrm{MT}=700 \quad \mathrm{Q}=-8.990 \mathrm{MeV} \quad \mathrm{EX}=0 . \quad \mathrm{MeV}$

$\begin{array}{lll}701 & -9.737 & 0.747\end{array}$

MT=738. (n,p) To Continuum States of ${ }^{15} \mathrm{C}$

Continuum defined to begin at $0.85 \mathrm{MeV}$, just above first excited state.

$M T=720-722$. (n,d) Cross Section to ${ }^{14} \mathrm{C}$ Ground, First, and Second Excited States 


$\begin{array}{rccc}\text { MT=720 } & Q=-7.984 & \mathrm{MeV} & \text { EX }=0 . \quad \mathrm{MeV} \\ 721 & -14.079 & & 6.095 \\ 722 & -14.567 & & =6.583\end{array}$

MT=738. (n,d) To Continuum States of ${ }^{14} \mathrm{C}$

Continuum defined to begin at $6.681 \mathrm{MeV}$, just above second excited level in ${ }^{14} \mathrm{C}$.

$M T=740-742 . \quad(n, t)$ Cross Section to ${ }^{13} \mathrm{C}$ Ground, First and Second Excited States

$\begin{array}{rcc}M T=740 & \mathrm{Q}=-9.902 \mathrm{MeV} & \mathrm{EX}=0 . \\ 741 & -12.992 & 3.09 \\ 742 & -13.586 & 3.684\end{array}$

MT=758. (n,t) To Continuun States of ${ }^{13} \mathrm{C}$

Continuum begins at $3.784 \mathrm{MeV}$, above second excited level in ${ }^{13} \mathrm{C}$. $\mathrm{MT}=780-785$. $(n, \alpha)$ Cross Sections to ${ }^{12} \mathrm{~B}$ Ground and First Five Excited Excited States

$\begin{array}{rrc}\text { MT=780 } & \text { Q=-7.623 MeV } & \text { EX=0. } \\ 781 & -8.576 & 0.953 \\ 782 & -9.297 & 1.674 \\ 783 & -10.244 & 2.621 \\ 784 & -10.343 & 2.72 \\ 785 & -11.013 & 3.39\end{array}$

MT=798. (n, $\alpha)$ To Continuum States of ${ }^{12} B$

Continuum begins at $3.49 \mathrm{MeV}$, just above fifth excited state in ${ }^{12} \mathrm{~B}$.

File 4. Secondary Neutron and Charged Particle Angular Distributions

MT=2. Elastic Angular Distributions

Zero to $5.4 \mathrm{MeV}$, based on results obtained from R-matrix theory analysis.

6 to $20 \mathrm{MeV}$, based on optical model calculation

$M T=16$. Angular Distribution for $(n, 2 n)$ Reaction

In the absence of data, isotropy in the laboratory system is assumed. $\mathrm{MT}=22$. Angular Distribution of Neutrons from $(n, n \alpha)$

Reaction assumed isotropic in the laboratory system. 
MT=28. Angular Distribution of Neutrons from $(n, n p)$ Reaction Assumed isotropic in the laboratory system.

MT=51-57. Angular Distribution Calculated Using Hauser-Feshbach Theory . $\mathrm{MT}=718$. Angular Distribution of Protons from $(n, p)$ Reaction Assumed isotropic in the laboratory system. $\mathrm{MT}=719$. Angular Distribution of Protons from (n,np) Reaction Assumed isotropic in the laboratory system. $M T=738$. Angular Distribution of Deutrons from (n,d) Reaction Assumed isotropic in the laboratory system. MT=758. Angular Distribution of Tritons from $(n, t)$ Reaction Assumed isotropic in the laboratory system. $M T=798$. Angular Distribution of Alphas from $(n, \alpha)$ Reaction Assumed isotropic in the laboratory system. $\mathrm{MT}=799$. Angular Distribution of Alphas from $(\mathrm{n}, \mathrm{n} \alpha)$ Assumed isotropic in the laboratory system.

File 5. Energy Distribution of Secondary Neutrons and Charged Particles MT=16, 22, 28, 91, 718, 758, and 798. Calculated with Statistical Model Code, GNASH (Yo77).

$M T=719$. Used for Proton Spectra from $(n, n p)$ Reaction

$M T=799$. Used for Alpha Spectra from $(n, n \alpha)$ Reaction

File 13. Photon Production Cross Sections

$\mathrm{MT}=4,16,103,104,105$ and 107, $\left(\mathrm{n}, \mathrm{n}^{\prime} \gamma\right),(\mathrm{n}, 2 \mathrm{n} \gamma),(\mathrm{n}, \mathrm{p} \gamma),(\mathrm{n}, \mathrm{d} \gamma)$, $(n, t \gamma),(n, \alpha \gamma)$ Cross Sections

Include both discrete and continuum contributions and were generated in the statistical model calculation with the GNASH code (Yo77).

File 14. Photon Angular Distributions

$\mathrm{MT}=4$. $\left(n, \mathrm{n}^{\prime} \gamma\right)$ to ${ }^{15} \mathrm{~N}$, all isotropic

$\mathrm{MT}=103$. ( $\mathrm{n}, \mathrm{pr}$ ) to ${ }^{15} \mathrm{C}_{9}$ all isotropic 


$$
\begin{aligned}
& \mathrm{MT}=104 . \quad(\mathrm{n}, \mathrm{d \gamma}) \text { to }{ }^{14} \mathrm{C}, \text { all isotropic } \\
& \mathrm{MT}=105 . \quad(\mathrm{n}, \mathrm{t \gamma}) \text { to }{ }^{13} \mathrm{C}, \text { all isotropic } \\
& \mathrm{MT}=107 . \quad(\mathrm{n}, \alpha \gamma) \text { to }{ }^{12} \mathrm{~B}, \text { all isotropic } \\
& \mathrm{MT}=16 . \quad(\mathrm{n}, 2 \mathrm{n} \gamma) \text { to }{ }^{14} \mathrm{~N}, \text { all isotropic }
\end{aligned}
$$

File 15. Energy Distribution of Secondary Photons

MT $=4,16,103,104,105,107$. Generated using statistical model code GNASH (Yo77).

\section{REFERENCES}

Aj70 F. Ajzenberg-Selove, Nuc1. Phys. A152, 1 (1973).

Aj71 F. Ajzenberg-Selove, Nuc1. Phys. A166, 1 (1971).

Ax62 L. P. Axe1, Phys. Rev. 126, 671 (1972).

Cl71 C. K. Cline and M. Blann, Nucl. Phys. Al72, 225 (1971).

Fe67 P. Fessenden and D. R. Maxson, Phys. Rev. 158, 948 (1967).

Gi65 A. Gillert and A. G. L. Cameron, Can. J. of Phys. 43, 1446 (1965).

Pe74 C. M. Perey and F. G. Perey, Atomic and Nucl. Data Tables 13, 293 (1974).

Si62 C. P. Sikkema, Nuc1. Phys. 32, 470 (1962).

U71 M. Uh1, Acta Physica Austria 31, 245 (1971).

Yo77 P. G. Young and E. D. Arthur, LA-6947 (1977),

Ze71 B. Zeitnitz et a1., Nuc1. Phys. Al66, 443 (1971). 
SUMMARY DOCUMENTATION FOR ${ }^{16} 0$

\author{
by \\ P. G. Young, D. G. Foster, Jr., and G. M. Hale \\ Los Alamos Scientific Laboratory \\ Los Alamos, New Mexico
}

\title{
I. SUMMARY
}

The ENDF/B-V evaluation for ${ }^{16} \mathrm{O}$ (MAT 1276) is based upon the Version IV evaluation. The only change made from the Version IV data was to update the formats for the correlated error information in File 33. The evaluation covers the energy range $10^{-5} \mathrm{eV}$ to $20 \mathrm{MeV}$ and is partially documented in LA-5759-PR (1974). See also LA-5375-PR (1973) and LA-4780 (1972).

\section{ENDF/B-V FILES}

File 1. General Information

MT=451. Descriptive Data.

Thinning Note: Cross section data in $M F=3$ and 13 for $M T=4,51-89$, $103,104,107,780-783$ were thinned above $E_{n}=6 \mathrm{MeV}$ using the requirement that interpolated values between any two points lie within $2 \%$ of the fine-grid value, providing that a certain basic grid $\left(200 \mathrm{keV}\right.$ intervals below $E_{\mathrm{n}}=10 \mathrm{MeV}$ and $500 \mathrm{keV}$ above $E_{\mathrm{n}}=10$ $\mathrm{Me} /$ ) be maintained. Below $\mathrm{E}_{\mathrm{n}}=6 \mathrm{MeV}$, a more stringent requirement of $1 \%$ was imposed on MT=780 and 107 .

In a similar manner, the $\mathrm{MT}=2$ Legendre coefficients in $\mathrm{MF}=4$ were thinned with the requirement that the interpolated angular distribution have an rms deviation from the fine-grid set of less than $2.5 \%$ and that the maximum excursion at any angle be less than $5 \%$.

\section{File 2. Resonance Parameters}

$M T=151$. Effective scattering radius $=0.54614 \times 10^{-12} \mathrm{~cm}$.

Resonance parameters not included.

File 3. Neutron Cross Sections

The $2200 \mathrm{~m} / \mathrm{s}$ cross sections are as follows:

$$
\mathrm{MT}=1 \quad \text { Sigma }=3.7483 \mathrm{~b}
$$


MT $=2 \quad$ Sigma $=3.7481 \mathrm{~b}$

$\mathrm{MT}=102$ Sigma $=0.1780 \mathrm{mb}$

MT $=1$. Total Cross Section

0.0 to $5.7 \mathrm{MeV}$, essentially calculated from R-Matrix parameters obtained by fitting simultaneously almost all available data for reactions ${ }^{16} \mathrm{o}(\mathrm{n}, \mathrm{n}){ }^{16} \mathrm{O},{ }^{16} \mathrm{o}\left(\mathrm{n}, \alpha_{0}\right)^{13} \mathrm{c},{ }^{13} \mathrm{c}\left(\alpha_{0}, \alpha_{0}\right){ }^{13} \mathrm{c}$ at energies below $E_{n}=5.7 \mathrm{MeV}$. Data for total are smoothed composite of Sc71 and normalized $\mathrm{Ci} 68$, with inserts of $\mathrm{Ci} 68$, normalized composite Fo73, and normalized Fo6l for narrow structure. Fo73 and Fo61 energy scales adjusted to match Ci68. Resolution corrections applied simultaneously to total and $\left(n, \alpha_{0}\right)$ to give same width and consistent heights to peaks. Level scheme is based on Jo73. 0.44-MeV resonance from normalized data of 0k55. Inserts from the smoothed composite are used over some narrow resonances, and over regions where it appears to better represent the experimental cross section. ${ }^{16} \mathrm{O}(\mathrm{n}, \gamma)$ capture cross section has been added at low energies, and the $n-{ }^{18} 0$ total cross section added in the vicinity of the $n-{ }^{16} 0$ minimum at $E_{n}=2.35 \mathrm{MeV}$.

5.7 to $20.0 \mathrm{MeV}$, based on a smoothed, empirical fit to the composite of experimental data described above.

$\mathbb{M T}=2$. Elastic Scattering Cross Section

0.0 to $5.7 \mathrm{MeV}$, R-matrix calculations.

6 to $11 \mathrm{MeV}$, obtained by subtracting the sum of MT=4, 102, 103, 104, and 107 from $M T=1$. However, adjustments were made to certain reaction channels, particularly MT $=51$, to enhance agreement with the elastic measurements of $\mathrm{Ph} 61, \mathrm{Ch} 61$, and especlally Ne72, Ki72, and Bu73.

11 to $14 \mathrm{MeV}$, smooth curve drawn so as to agree with $1.4-\mathrm{MeV}$ measurements of Ba63, Mc66, and Be67.

14 to $20 \mathrm{MeV}$, optical model extrapolation using parameters from fit of 14-MeV data of Ba63, adjusted to give correct total cross sections up to $20 \mathrm{MeV}$.

MT=4. Inelastic Cross Section

Threshold to $20 \mathrm{MeV}$, sum of $\mathrm{MT}=51-89$.

MT=51-70. Inelastic Cross Section to Discrete States

$\begin{array}{rrrrrr}\text { MT=51 } & \mathrm{Q}=-6.052 \mathrm{MeV} & \mathrm{MT}=58 & \mathrm{Q}=-10.354 \mathrm{MeV} & \mathrm{MT}=65 & \mathrm{Q}=-11.63 \mathrm{MeV} \\ 52 & -6.131 & 59 & -10.952 & 66 & -12.053 \\ 53 & -6.917 & 60 & -11.080 & 67 & -12.442 \\ 54 & -7.119 & 61 & -11.096 & 68 & -12.528 \\ 55 & -8.872 & 62 & -11.26 & 69 & -12.795 \\ 56 & -9.597 & 63 & -11.44 & 70 & -12.967 \\ 57 & -9.847 & 64 & -11.521 & & \end{array}$


Threshold to $20 \mathrm{MeV}, \mathrm{MT}=52-55,59$, and 60 are based mainly on $(\mathrm{n}, \mathrm{n} \gamma)$ data of D170, Or70, Dr70, Bu71, Ny69, Cl69, and En64 below $15 \mathrm{MeV}$ and were extrapolated to $20 \mathrm{MeV}$ with compound nucleus reaction theory calculations. The remaining MT numbers are based on compound nucleus calculations normalized to give an $M T=3$ in agreement with elastic data of Ba62, Be67, and Mc66 at $14 \mathrm{MeV}$. At lower energies, adjustments made, especially to $\mathrm{MT}=51$, to enhance agreement with $\left(n, n^{\prime}\right)$ measurements of $\mathrm{Ne} 72, \mathrm{Ki} 72$ and with elastic measurements of $\mathrm{Ne} 72, \mathrm{Ki} 72$, and Bu73. Integral data from sphere transmission measurements (Wo72) were also factored into deriving some of the $14-\mathrm{MeV}$ data.

MT=71-89. Inelastic Cross Section to Groups of Discrete Stat॰s

Data combined into $0.3-\mathrm{MeV}$ wide excitation energy bins centered about Q-values between -13.15 MeV (MT=71) and -18.55 MeV (MT=89). This representation was used in lieu of $M F=5, M T=91$, which does not preserve kinematic relationships.

Threshold to $20 \mathrm{MeV}$, integrated cross section adjusted to give a nonelastic cross section in agreement with the evaluated total and elastic. The cross section was divided among bands according to a nuclear temperature calculation using $\mathrm{T}=2 \mathrm{MeV}$. Please note that much of the cross section to levels above $9 \mathrm{MeV}$ subsequently results in charged particle emission. MT=56-58, 61-66, 68-70, $72-73,75,77-78,80,82-83,85,87$, and 89 , are flagged as decaying by alpha emission. This choice leads to a total alpha emission cross section simflar to data of Li.52, Da68, anc Bo66 below $17 \mathrm{MeV}$. The remaining MT numbers above MT=66 are flagged as being proton emitters.

$\mathrm{MT}=102 . \quad(\mathrm{n}, \gamma)$ Cross Section

$1 / V$ variation from $178 \mu \mathrm{b}$ at $2200 \mathrm{~m} / \mathrm{s}$, from Ju64.

MT=103. (n,p) Cross Section

Threshold to $15 \mathrm{MeV}$, used evaluation of S165, raised 5 per cent above $12 \mathrm{MeV}$.

15 to $20 \mathrm{MeV}$, based on experimental data of Bo66, Se62, De62, and Ma54.

$M T^{\prime}=104 . \quad(n, d)$ Cross Section

Threshold to $20 \mathrm{MeV}$, compound nucleus reaction theory calculation normalized to single datum point of Li52 at $14 \mathrm{MeV}$.

MT=107. $\quad(n, \alpha)$ Cross Section

Threshold to $20 \mathrm{MeV}$, sum of $\mathrm{MT}=780-783$. 
$\mathrm{MT}=780 . \quad(n, \alpha)$ Integrated Cross Section to ${ }^{13} \mathrm{C}$ Ground State

0.0 to $5.7 \mathrm{MeV}$, essentially calculated from R-matrix fit described under MT=1. Energy scale of Ba72 adjusted to match Ci68 total cross section and resolution corrections applied to give width and height of peaks consistent with total. Normalization of 0.85 for Ba72 determined from R-matrix fit and applied. Level scheme based on Jo73.

5.7 to $20 \mathrm{MeV}_{3}$, based on data of Da63, Da68, Si68, Ba73, and composite of Mc66A, Ma68, and Le68 at $14 \mathrm{MeV}$.

$\mathrm{MT}=781-783$. ( $n, \alpha)$ Cross Section to Excited Levels of ${ }^{13} \mathrm{C}$

Threshold to $15 \mathrm{MeV}$, used $(n, \alpha)$ data of Da63 and $(n, \alpha \gamma)$ data of Di70, Or70, C169, Ny69, En64, and Bu71.

15 to $20 \mathrm{MeV}$, based on data of Si68.

File 4. Neutron Angular Distributions

MT $=2$. Elastic Angular Distributions

0.0 to $5.7 \mathrm{MeV}$, calculated from R-matrix fit (see $M F=3, M T=1$ ). Measured angular distributions input to the fit were those of Ch61, Fo58, Fo70, Hi58, Hu62, Jo67, Ki72, La60, Li66, Ma62, and Ph61.

5.7 to $14 \mathrm{MeV}$, smooth curve through coefficients derived from fits to elastic data of $\mathrm{Ph} 61, \mathrm{Ne} 72, \mathrm{Ch} 61, \mathrm{Ba} 63, \mathrm{Be} 67, \mathrm{Mc66}, \mathrm{Ki72}$, and Bu73.

14.0 to $20 \mathrm{MeV}$, from optical model calculation using parameters from fic to $14 \mathrm{MeV}$ data of $\mathrm{Ba63}$, adjusted to give correct total cross sections up to $20 \mathrm{MeV}$.

MT=51-89. Inelastic Angular Distributions

Threshold to $20 \mathrm{MeV}$, assumed isotropic in center-of-mass system except for MT=51-55 above $10 \mathrm{MeV}$ which are based on fits of the 14-MeV measurements of Ba63 and Mc66. Note that the sum MT=51 and 52 is consistent with isotropy at $8.56 \mathrm{MeV}$ (Ki72).

File 7. Thermal Scattering Low Data

Provided by D. Finch (SRL). Constrained to match $M F=3, M T=2$ data.

File 12. Photon Multiplicities

MT=102. Radiative Capture

Based on experimental data from Ju64 and private communication. 


\section{File 13. Gamma Ray Cross Sections}

\section{$M T=4 . \quad(r, n \gamma)$ Cross Sections}

Threshold to $15 \mathrm{MeV}$, based mainly on data of Di70, 0r70, Dr70, Lu70, Bu69, Ny69, Cl69, and En64. Data generated from MF=3 $\mathrm{MT}=51-60$ using an ${ }^{16} \mathrm{O}$ decay scheme from $\mathrm{Aj} 70$.

15 to $20 \mathrm{MeV}$, based on extrapolation of $\mathrm{MT}=51-60$ using compound nucleus reaction theory calculations.

Note that the first excited level of ${ }^{16} \mathrm{O}$ at $6.052 \mathrm{MeV}$ is assumed to decay with emission of two $0.51-\mathrm{MeV}$ gamma rays.

MT=22. (n, n $\alpha \gamma)$ Cross Sections

Threshold to $20 \mathrm{MeV}$, smooth curve based crudely on $(n, n \alpha)$ cross section and known levels in ${ }^{12} \mathrm{C}$, adjusted to agree with composite of $14-\mathrm{MeV}$ data of $\mathrm{C} 169$, and $0 r 70$.

MT=103. (n,pr) Cross Sections

Threshold to $20 \mathrm{MeV}$, based on crude division of $\mathrm{MF}=3$, MT=103 cross section among available levels according to $(2 * \mathrm{~J}+1)$.

$M T=107 . \quad(n, \alpha \gamma)$ Cross Sections

Threshold to $15 \mathrm{MeV}$, based mainly on ( $n, \alpha \gamma$ ) data of Di70, or70, Cl69, Ny69, En64, Bu71, and $(n, \alpha)$ data of Da63.

15 to $20 \mathrm{MeV}$, based on data of Si68.

File 14. Gamma Ray Angular Distributions

$\mathrm{MT}=4$. $\quad(\mathrm{n}, \mathrm{n} \gamma)$ Angular Distributions

Assumed isotropic except for the 6.131-MeV gamma, which is based on the angular distribution measurement of $\operatorname{Dr} 70, \mathrm{Lu} 70, \mathrm{Bu} 71$, and $\mathrm{D} 170$, and the $6.917-\mathrm{MeV}$ gamma, which is based on the two-angle measurements of Di70.

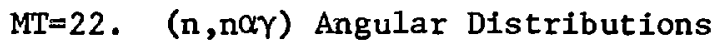

Assumed isotropic.

$M T=103 . \quad(n ; \mathrm{p} \gamma)$ Angular Distributions

Assumed isotropic.

$\mathrm{Mr}=107$. (n, ar) Angular Distributions

Assumed isotropic. 
File 33. Neutron Cross Section Covariances

$\mathrm{MT}=1,2,4,103,107$. Smooth Cross Section

Covariances are based upon estimates of the uncertainty in the experimental measurements and theoretical calculations used in the evaluation. Format updated for Version-V. For more details, see separate summary documentation for covarlances.

\section{REFERENCES}

Ad49 R. K. Adair et al., Phys. Rev. 75, 1124 (1949).

Aj70 F. Ajzenberg-Selove, Nuc1. Phys. A52, 1 (1970).

Ba63 R. W. Bauer et al., Nuc1. Phys, 47, 241 (1963).

Ba73 J. K. Batr and F. X. Haas, Phys. Rev. C7, 1356 (1973).

$\left(n, \alpha_{0}\right)$ cross sections obtained by detail balance from this reference.

Be67 P. L. Beach et al., Phys. Rev. 156, 1201 (1967).

Bo66 M. Bormann et al., Proc. IAEA Conf. Nucl. Data, Paris (1966), p.225.

Bu71 P. S. Buchanan et al., OR0-2791-32 (1971).

Bu73 W. P. Bucher et al., BRL-R-1652 (1973).

Ch61 L. F. Chase et al., AFSWC-TR-61-15 (1961).

Ci68 S. Cierjacks et al., KFK-1000 (1960).

Cl69 G. Clayeux and G. Grenier, CEA-R-3807 (1969).

Da63 E. A. Davis et al., Nuc1. Phys. 48169 (1963).

Da68 D. Dandy et al., AWRE 060/68 (1968).

De62 J. A. DeJuren et al., Phys. Rev. 127, 1229 (1962).

Di70 J. K. Dickens and F. G. Perey, Nucl. Sc1. Eng. 40, 283 (1970).

Dr70 D. M. Drake et al., Nucl. Sc1. Eng. 40, 294 (1970).

En64 F. C. Engesser and W. E. Thompson, J. Nuc1. Eng. 21, 487 (1967).

Fo58 J. L. Fowler and H. O. Cohn, Phys. Rev. 109, 89 (1958).

Fo61 D. B. Fossan et al., Phys. Rev. 123, 209 (1961).

Fo 70 J. L. Fowler and C. H. Johnson, Phys. Rev. C2, 124 (1970).

Fo73 J. L. Fowler et al., Phys, Rev. C8, 545 (1973).

Hi58 R. W. Hill, Phys. Rev. 109, 2105 (1959).

Hu62 W. Hunzinger and P. Huber, Helv. Phys. Acta 35, 351 (1962).

Jo48 W. B. Jones, Jr., Phys. Rev. 74,364 (1948).

Jo67 C. H. Johnson and J. L. Fowler, Phys. Rev. 162, 890 (1967).

Jo73 C. H. Johnson, Phys. Rev. C7, 561 (1973).

Ju64 E. T. Jurney and H. T. Motz, Bull. Am. Phys. Soc. 9, 176 (1964).

Ki72 W. E. Kinney and F. G. Perey, ORNL-4760 (1972).

La60 $R .0$. Lane et al., ANL-6172 (1960).

Le68 B. Leroux et al., Nucl. Phys. A116, 196 (1968).

Li52 A. B. Lillie, Phys. Rev. 87, 716 (1952).

Li66 D. Lister and A. Sayres, Phys. Rev. 143, 745 (1966).

Lu70 B. Lundberg et al., Physica Scripta 273 (1970).

Ma54 H. C. Martin, Phys. Rev. 93, 498 (1954).

Ma62 J. P. Martin and M. S. Zucker, Bull. Am. Phys. Soc. I, 72 (1962).

Ma68 D. R. Maxson and R. D. Murphy, Nuc1. Phys. A110, 555 (1968).

Mc66 W. J. McDonald et al., Nuc1. Phys. 75, 353 (1966).

Mc66A W. N. McDonald and W. Jack, Nucl. Phys. 88, 457 (1966).

Me49 E. Melkonian, Phys. Rev. 76, 1750 (1949).

Ne71 D. O. Nellis and P. S. Buchanan, private communication (1971). 
Ne72 D. 0. Nel11s and P. S. Buchanan, DNA-2716 (1972).

Ny69 K. Nyberg, private communication to L. Stewart (1969).

Ok55 A. Okazaki, Phys, Rev. 99, 55 (1955).

Or70 V. J. Orphan et al., Nuc1. Sci. Eng. 42, 352 (1970).

Ph61 D. D. Phillips, private communication to BNL (1961).

Sc71 R. B. Schwartz, private communication (1971).

Se62 K. W. Seemann and W. E. Moore, KAPL-2214 (1962).

Siss Von I. Sick et al., Helv. Phys. Acta 41, 573 (1968).

Sl65 E. L. Slaggie and J. T. Reynolds, KAPL-M-6452 (1965).

Wo72 C. Wong et al., UCRL-51144, Rev. 1 (1972). 


\title{
SUMMARY DOCUMENTATION FOR $27_{\text {Al }}$
}

by

\author{
P. G. Young and D. G, Foster, Jr. \\ Los Alamos Scientific Laboratory \\ Los Alamos, New Mexico
}

\section{SUMMARY}

The ENDF/B-IV evaluation for ${ }^{27} \mathrm{Al}$ was carried over for Version $\mathrm{V}$ as MAT 1313. Besides minor format changes, the only new data included are correlated errors in File 33. The evaluation covers the energy range $10^{-5} \mathrm{eV}$ to $20 \mathrm{MeV}$. Documentation for the evaluation is LA-4726 (1972), as updated by LA-5759-PR (1974).

\section{ENDF/B-V FILES}

File 1. General Information

MT=451. Descriptive data.

File 2. Resonance Parameters

$\mathrm{MT}=151$. Effective scattering radius $=0.32752 \times 10^{-12} \mathrm{~cm}$.

Resonance parameters not given.

\section{File 3. Neutron Cross Sections}

The $2200 \mathrm{~m} / \mathrm{s}$ cross sections are:

$$
\begin{array}{ll}
\mathrm{MT}=1 & \sigma=1.580 \mathrm{~b} \\
\mathrm{MT}=2 & \sigma=1.348 \mathrm{~b} \\
\mathrm{MT}=102 & \sigma=0.232 \mathrm{~b}
\end{array}
$$

MT=1. Total cross Section

Below $4.6 \mathrm{keV}$ : $1 / \mathrm{V}$ fit to Me52 and Hi50 (normalized to Me52), using thermal capture $\sigma$ of $232 \mathrm{mb}$ (Go71) and resulting in a total of $1.580 \mathrm{~b}$.

4.6 - $189 \mathrm{keV}$ : From Ga65 normalized to Me52 via Hi59 at low energies and to $\mathrm{Pe} 72$ at high energies.

209 - $475 \mathrm{keV}$ : From Pe72 with energy scale corrected to match $\mathrm{C} 168$ at higher energies. 
.475 - $11.5 \mathrm{MeV}$ : Composite of Pe72 and Sc69, normalized to their weighted average, with inserts of normalized Ci68 where needed to preserve resolution.

11.5 - $20 \mathrm{MeV}$ : Composite of Pe72, Fo71, Al66, and Pe60 normalized to their weighted average. Slope at $20 \mathrm{MeV}$ includes guidance from Ta55.

Smoothing between $4.6 \mathrm{keV}$ and $2 \mathrm{MeV}$ by approximate Breit-Wigner fits where possible, connected by polynomial fits. $2-12 \mathrm{MeV}$ smoothed by running cubic polynomial fits. Above $12 \mathrm{MeV}$ fitted secondary polynomial to middles of running polynomial fits.

MT=2. Elastic Scattering Cross Section

Below $5 \mathrm{MeV}$ : Subtracted nonelastic from total.

5 to $16 \mathrm{MeV}$ : Mainly based on elastic data of Ho69, St59, Ki70, St65, Co58, Co59, Be58, Mi68, Be56, and Ka72 together with the evaluated total and the nonelastic measurements iisted below.

16 to $20 \mathrm{MeV}$ : Smooth extrapolation to $1 / 2$ the total at $20 \mathrm{MeV}$.

MT=3. Nonelastic Cross Section (implicit - not in file)

Below $9 \mathrm{MeV}$ : Based on the nonelastic measurements of Ba58, Be56, Ta55, De65, on the sum of MT=4,102, 103, 107, and on the difference between the evaluated total and the elastic neasurements of $\mathrm{Ho69}, \mathrm{Ki} 70, \mathrm{Be} 56$, and Mi68.

9 to $16 \mathrm{MeV}$ : Based on nonelastic measurements of Ba58, Ta55, Gr55, $\mathrm{Ph} 52, \mathrm{Ma57}, \mathrm{De} 61, \mathrm{~F} \ell 56, \mathrm{Ch} 67$, and on the difference between $M T=1$ and $M T=2$ data of $S t 59, S: 65, C o 58, C o 59$, and Be58.

16 to $20 \mathrm{MeV}$ : Difference between $\mathrm{MT}=1$ and $\mathrm{MT}=2$.

MT=4. Inelastic Cross Section

Threshold to $5 \mathrm{MeV}$ : Sum of $\mathrm{MT}=51-63$.

5 to $9 \mathrm{MeV}$ : Based on $\left(n, n^{\prime}\right)$ data of Th63, To67, the evaluated $\left(n, n^{\prime}\right)$ data of Di7l which includes consideration of several other measurements, and the $(n, n Y)$ data of $D i 71,0 r 71$, and Di73.

9 to $20 \mathrm{MeV}$ : The difference between $\mathrm{MT}=1$ and the sum of $\mathrm{MT}=2,16$, $102,103,104,105$, and 107.

$\mathrm{MT}=16 . \quad(n, 2 n)$ Cross Section

Threshold to $20 \mathrm{MeV}$ : Estimated using a nuclear temperature calculation assuming that highly excited states in ${ }^{27} \mathrm{Al}$ decay 50 per cent by neutron emission. 
MT=51 - 63. Inelastic Cross Section to Discrete States

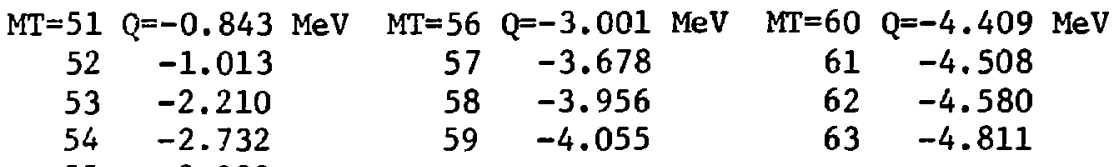

Threshold to $5 \mathrm{MeV}$ : Based on the $\left(n, n^{\prime}\right)$ data of To62, Wi63, Ts61, and the $(n, n \gamma)$ data of Ch68, Ma65, Di71, Or71, and Di73.

5 to $9 \mathrm{MeV}$ : Based on an evaluation of several measurements given in Di71 (Table B1).

9 to $20 \mathrm{MeV}$ : Smooth extrapolation passing through $14 \mathrm{MeV}$ data of Stó 5 , and Bo65A.

MT=64 - 90. Inelastic Cross Section To Groups of Discrete States in Energy Bins Centered About Q-values Between $-5.25 \mathrm{MeV}$ and $-18.875 \mathrm{MeV}$.

Threshold to $20 \mathrm{MeV}$ : Integrated cross section over ìands obtained by subtracting $M T=51-63$ from $M T=4$. Cross section divided antong bands according to a nuclear temperature calculation using tamperatures based on $\left(n, n^{\prime}\right)$ data of Th63, and Gr53. The cross section to the bands with $M T=64-71$ was adjusted extensively to produce agreement with the $14-\mathrm{MeV}$ measurements of secondary neutron spectra by Ka72.

(Please note that much of the cross section to bands above $E_{X}=9 \mathrm{MeV}$ subsequently results in charged particle emission. Since these data are not included explicitly as $(n, n x)$ reactions, it is important that users involved in certain calculations (e.g., local heating) be aware of this information.)

MT $=102 . \quad(n, \gamma)$ Cruss Section

Below $1 \mathrm{keV}$ : $1 / \mathrm{V}$ from thermal value of $232 \mathrm{mb}$ (Go71).

1 to $140 \mathrm{keV}$ : From B168, 6-keV resonance has width deduced from total cross section. Small resonances nearby also from total.

Above $140 \mathrm{keV}$ : Mainly from He50, He53, and Ca62.

$\mathrm{MT}=103$. (n,p) Cross Section (see Yo72 for more details)

Threshold to $4 \mathrm{MeV}$ : Based on the measurements of $\mathrm{He} 54$ and $\mathrm{Gr67}$, extrapolated from $3 \mathrm{MeV}$ to threshold with an L=0 penetrability function for the outgoing $p+{ }^{27} \mathrm{Mg}$ channel.

4 to $20 \mathrm{MeV}$ : Smooth curve through the measurements of He54, Gr67, $\mathrm{C} a 62, \mathrm{Ba} 66$, and Ma60. 
MT=104. (n,d) Cross Section

Threshold to $20 \mathrm{MeV}$ : Smooth curve through single datum of Gl61.

$\mathrm{MT}=105 . \quad(n, t)$ Cross Section

Threshold to $20 \mathrm{MeV}$ : Smooth curve with same shape as MT=104.

Reaching maximum of $15 \mathrm{mb}$ at $20 \mathrm{MeV}$.

$\operatorname{MT}=107 .(n, \alpha)$ Cross Section (see Yo72 for more details)

Threshold to $20 \mathrm{MeV}$ : Based on a smooth curve through experimental data, mainly those of Bu63, Pa65, and Li66, with results from Gr67, Sc61, Gr58, Ba61, Ma65, Ga62, Im64, Ke59 and several 14-MeV points also being considered. The curve was extrapolated from 6 $\mathrm{MeV}$ to threshold with an $\mathrm{L}=0$ penetrability function for the outgoing $\alpha+{ }^{24} \mathrm{Na}$ channel.

File 4. Neutron Angular Distributions

MT=2. Elastic Angular Distributions

From smooth curves through plots of coefficlents from fits to all available data above $.25 \mathrm{MeV}$, without evaluation of measurements. Mainly from Ta57, Ch66, To62, Ts61, Ki70, and Be58, with opticalmodel bridge to Si62. Some of data augmented before fitting by zero-degree point slightly above Wick limit, and coefficients adjusted empirically afterwards to obey Wick limit. But only barely at higher energies.

$M T=16$. (n,2n) Angular Distributions

Assumed isotropic in CM, using 2-body kinematics to estimate transformation to laboratory system.

MT=51 - 63. Inelastic Neutron Angular Distributions

Anisotropic distributions based on measurements by Ts61, To62, Wi63, Mi68, Ta70, Gl72, St65, Bo65A, and Ka72 were incorporated over varying energy ranges from threshold to $20 \mathrm{MeV}$. In several regions where data were lacking, isotropy was assumed.

MT=64 - 90. Inelastic Neutron Angular Distributions

Assumed isotropic in the center-of-mass system.

File 5. Neutron Energy Distributions

MT=16. $(n, 2 n)$ Energy Distributions

Based on statistical theory. 
File 12. Gamma Ray Multiplicities

MT $=102, \quad(n, \gamma)$ Capture Multiplicities

The ${ }^{28} \mathrm{~A} 2$ decay scheme for excitation by radiative capture with thermal neutrons from Ha69 was adopted after some modification to enhance agreement with experiment. The results have been compared with the less detailej data of Ba67, Ju71, and Ma69, and are reasonably consistent. Some of the lines in Ra70 below $1 \mathrm{MeV}$ appear to be spurious and have been dropped. Spectrum for thermal neutrons is assumed to apply at all energies.

File 13. Gamma Ray Smooth Cross Sections

$M T=4 . \quad(n, n \gamma)$ Cross Sections

Threshold to $5 \mathrm{MeV}$, based on the $\left(n, n^{i}\right)$ data of To62, Wi63, Ts61, and the $(n, n \gamma)$ data of Ch68, Ma65, Di71, Or71, and Di73.

5 to $20 \mathrm{MeV}$. Mainly from (n,ny) data of $\mathrm{Di} 73$, Or71, and $\mathrm{Di} 71, \mathrm{Dr} 73$, Pe64, Be66, Cl69, En67, Bo65, Ma68, Ca60 and Pr60, supplemented by statistical theory calculations.

$M T=28 . \quad(n, n p \gamma)$ Cross Sections

Threshold to $20 \mathrm{MeV}$, based on the measurements of $\mathrm{Di} / 3$, Or71, En67, Pr60, C169, Be66, Bo65, and on statistical theory.

MT=103. (n, PY) Cross Sections

Threshold to $9 \mathrm{MeV}$, used data of Di71.

9 to $20 \mathrm{MeV}$. Smooth Extrapolation.

File 14. Gamma Ray Angular Distributions

$\mathrm{MT}=4$. (n, nY) Angular Distributions Assumed Isotropic.

$M T=28$. ( $n, n p \gamma)$ Angular Distributions Assumed Isotropic.

$M T=103$. ( $n, p \gamma$ ) Angular Distributions Assumed Isotropic.

File 15. Gamma Ray Energy Distributions

MT=4. (n,n $\gamma)$ Energy Distributions

Statistical theory calculation adjusted to fit measurement of Di73. MT=28. (n,npY) Energy Distributions

Statistical theory calculation adjusted to fit measurement of Di73. 


\section{File 33. Neutron Crcss Section Covariances}

Based on revised version of Table 6 in LA-4726. Errors are given only for MT=1, 2, 4, 16, 102, 103, and 107. MT=2 is taken from experiment between 9 and $17 \mathrm{MeV}$, and derived evexywhere else. $\mathrm{MT}=4$ is derived above $9 \mathrm{MeV}$. The derivation formulas are given by NC-type sub-subsections with $L T Y=0$ in $M T=2$ and $M T=4$.

\section{REFERENCES}

Ba58 W. P. Ball et a1., (LRL), Phys. Rev. 110, 1392 (1958).

Ba61 B. P. Bayhurst and R. J. Prestwood, ( $\overline{\text { LAS }), ~ P h y s . ~ R e v . ~ 121, ~} 1438$ (196i).

Ba66 R. Base, EANDC(E)-66U, P. 64, and Private communication (1966).

Ba67 G. A. Bartholomew et al., Nuc1. Data A3, 367 (1967).

Be55 J. R. Beyster et al., (LAS), Phys. Rev. 98, 1216 (1955).

Be56 J. R. Beyster et al., (LAS), Phys. Rev. 104, 1319 (1956).

Be58 S. Berko et a1., Nuc1. Phys. 6, 210 (1958).

Be66 V. M. Bezotosnyi et al., (Rus), Sov. J. Nucl. Phys. 3, 632 (1966).

B168 R. C. Block, Private communication to R. J. Howerton (1968).

Bo59 N. A. Bostrom et al., (TNC), WADC-TN-59-107 (1959).

Bo65 V. N. Bockharev and V. V. Nepedov (LEB), Sov. J. Nucl. Phys. 1, 574 (1965).

Bo65A G. C. Bonazzola et al., (TUR), Phys, Rev. 140, 635 (1965).

Bu63 J. P. Butler and D. C. Santry, Can. J. Phys. 41, 372 (1963).

Ca60 R. L. Caldwell et al., (SOC), Nucl. Sci. Eng. 8 , 173 (1960).

Ca62 G. Calvi et a1., Nuc1. Phys. 39, 621 (1962).

Ca67 A. D. Carlson and H. H. Barschall, Phys, Rev. 158, 1142 (1967).

Ch66 J. P. Chien and A. B. Smith, Nucl. Sc1. Eng. $2 \overline{6}, 500$ (1966).

Ch67 A. Chatterjee and A. M. Ghose (BOS), Phys. Rev. 161, 1181 (1967).

Ch68 K. C. Chung et al., (KTY), Nuc1. Phys, 68, 476 (1968).

Ci68 S. Cierjacks et a1., KFK-1000 (1968).

Cl69 G. Clayeux and G. Grenier (FR), CEA-R-3807, (I969).

Co58 J. H. Coon et al., (LAS), Phys. Rev. 111, 250 (1958).

Co59 J. H. Coon (LAS), Private communications to R. J. Howerton (1959).

De61 Yu. G. Degtyarev and V. G. Nadtochii, Sov. J. At. Energy 11, 1043 (1961).

De65 Yu. G. Degtyarev (RUS), J. Nuc1. Energy 20, 818 (1965).

Di71 J. K. Dickens (CRL), ORNL-TM-3284, 1971.

Di73 J. K. Dickeins et al., ORNL-TM-4232 (1973).

Dr70 D. M. Drake et al., (LAS), Nuc1. Sc1. Eng. 40, 294 (1970).

En67 F. C. Engesser and W. E. Thompson (NRD), J. Nuc1. Energy 21, 487 (1967).

Fe67 J. M. Fergison and J. C. Albergotti (NRD), Nucl. Phys. 98, 65 (1967).

Fl56 N. N. Flerov and V. M. Talyzin (RUS), Atomnaya Energiya 1, 155 (1956).

Fo71 D. G. Foster, Jr. and D. W. Glasgow, Phys, Rev, C3, 576 (1971).

Ga62 F. Gabbard and B. D. Kern (KTY), Phys. Rev. 128, $\overline{12} 76$ (1962).

Ga65 J. B. Garg et a1., Private communication to R. J. Howerton (1965).

Gl61 R. N. Glover and E. Wilgold (CBR), Nuc1. Phys. 24, 630 (1961).

Gl72 D. W. Glasgow, Private communication (1972).

Go71 D. T. Goldman, Unpublished evaluation (1971).

Gr53 E. R. Graves and L. Rosen (LAS), Phys. Rev. 89, 343 (1953).

Gr55 E. R. Graves and R. W. Davis (IAS), Phys. Rev. 97, 1205 (1955).

Gr58 J. A. Grundl et al., (LAS), Phys. Rev. 109, 425 (1958).

Gró7 J. A. Grundl (LAS), Nucl. Sci. Eng. 30, 69 (1967).

Ha69 R. Harde11 et al., Nuc1. Phys. A126, 392 (1969). 
He50 R. L. Henkel and H. H. Barschall, Phys. Rev. R0, 145 (1950).

He53 R. L. Henkel, Private communication to Sigma Center (1953).

Hi59 C. T. Hibdon, Phys. Rev. 114, 179 (1959).

Ho69 B. Holmqvist and T. Wiedling (AE), AE-366 (1969).

Im64 W. L. Imhog (LOK), private communication to NNCSC (1964).

Jo64 G. D. Joanou and C. A. Stevens (GA), GA-5884 (1964).

Ju71 E. T. Jurney, Private communication (1971).

Ka72 J. L. Kammerdiener, Thesis, University of California at Davis (1972).

Ke59

$\mathrm{Ki} 70$

La57

Li66

Ma57

Ma60

Ma65

Ma68

Ma69

$\mathrm{Me} 52$

Mi68

0r71

Pa65

Pe64

$\mathrm{Pe} 72$

B. D. Kern et a1., (NRD), Nucl. Phys. 10, 226 (1959).

W. E. Kinney and F. G. Perey (ORL), ORNL-4516 (1970).

A. Langsdorf et a1., Phys. Rev. 107, 1077 (1957).

H. Liskien and A. Paulsen, Nucleonics $\underline{8}, 315$ (1966).

M. H. MacGregor et al., (LRL), Phys. Rev. 108, 726 (1957).

G. S. Mani et al., (HAR), Nucl. Phys. $19, \overline{535}$ (1960).

S. C. Mathur et a1., (TNC), Nuc1. Phys. 73, 561 (1965).

G. N. Maslov et a1., (RUS), Sov. J. At. Energy 24, 704 (1968).

R. E. Maerker and F. J. Muckenthaler, ORNL-4382 (1969).

A. W. Merrison and E. R. Wiblin, Proc. Roy. Soc. (L) 215, 278 (1952).

A. Mittler et al., (KTY), Bull. Am. Phys. Soc. 13, 1420 (1968).

V. J. Crphan and C. G. Hoot, Gulf-RT-A10743 (1971).

Ph52 D. D. Phillips et al., (LAS), Phys. Rev. 88, 600 (1952).

Pr60 J. T. Prudhomme et a1., (TNC), AFSWC-TR-60-30 (1960).

Ra70 N. C. Rasmussen et al., GA-10248 (1970).

Sc61 H. W. Schmitt and J. Halperin (ORL), Phys. Rev. 121, 827 (1961).

Sc70 R. B. Schwartz, private communication (1970).

St59 C. St. Pierre et al., (MON), Phys. Rev. 115, 999 (1959).

St62 T. P. Stuart et al., Phys. Rev. 125, 276 (1962).

St65 P. H. Stelson and R. L. Robinson (ORL), Nucl. Phys. 68, 97 (1965).

Ta55

TA70

Th63

To62

T067

Ts61

Wi63

H. L. Taylor et al., (RIC), Phys. Rev. 100, 174 (1955).

S. Tanaka et a1., Nuc1. Data for Reactors, IAEA p. 317 (1970).

D. B. Thompson (LAS), Phys. Rev. 129, 1649 (1963).

J. H. Towle and W. B. Gilboy, Nuc1. Phys. 39,300 (1962).

J. H. Towle and R. O. Owens (ALD), Nucl. Phys. A100, 257 (1967).

3 D. Winterhalter (ZAG), Nucl. Phys, 43, 339 (1963).

Yo72 P. G. Young, LASL Memo T-2-99 to the Norm. and Standards Subcommittee of CSEWG (1972). 
SUMMARY DOCURENTATION FOR ${ }^{182} \mathrm{~W}$

\author{
by \\ P. G. Young, J. Otter, ${ }^{\dagger}$ E. Ottewitte, ${ }^{\dagger}$ and P. Rose ${ }^{\dagger}$ \\ Los Alamos Scientific Laboratory \\ Los Alamos, New Mexico
}

\title{
I. SUMMARY
}

The ${ }^{182} \mathrm{~W}$ evaluation for ENDF/B-V (MAT 1128) was carried over intact from Version IV with only minor format changes being made. The evaluation of the neutron files was performed at Atomics International and is documented in TI-707-130-026 (1973). The gamma-ray production data were evaluated at Los Alamos Scientific Laboratory and are documented in LA-5793 (1975). The ENDF/B-V data span the energy range $10^{-5} \mathrm{eV}$ to $20 \mathrm{MeV}$.

\section{ENDF/B-V FILES}

File 1. General Information

MT=451. Descriptive Data

Atomic mass and Q-values taken from Ref. 1 .

File 2. Resonance Parameters

$\mathrm{MT}=151$. (A) Resolved Resonances Evaluation

Potential scattering cross section $=8.0 \pm 1.0$ b at $E=0$.

$$
\begin{aligned}
& 2200 \mathrm{~m} / \mathrm{s} \text { Cross Sections (barns) } \\
& \text { CAP CALC MEAS (Ref. 2) } \\
& \text { SCAT } 11.6
\end{aligned}
$$

Resolved Resonance Parameters

$\begin{array}{rll}-100 \mathrm{eV} & \text { from } & \text { fit to capture } \\ 4.16 \mathrm{eV} & \text { frcm } & \text { Ref. } 2 \\ 5-250 \mathrm{eV} & \text { from } & \text { evaluation, Refs. 2, 3, and } 4 \\ 250-1250 \mathrm{eV} & \text { from evaluation, Refs. 3 and } 4 \\ 1250-4500 \mathrm{eV} & \text { from } & \text { Ref. } 4 \\ 1920-2198 \mathrm{eV} & \text { from } & \text { Ref. } 3\end{array}$

$\varlimsup_{\text {Atomics International }}$ 
MT=151. (B) Unresolved Resonances Evaluation

Potential scattering cross section $=8.5 \pm 1.0 \mathrm{~b}$ at $E_{\mathrm{n}}=0$. Total cross section $=8.85 \mathrm{~b}$ at $\mathrm{E}_{\mathrm{n}}=100 \mathrm{keV}$ (calculated).

Unresolved resonance parameters from automated optimized fit to the evaluated measured capture cross section.

AV $L=0$ level spacing $\left(E_{n}=0\right)=57.45 \mathrm{eV}$, energy dep. fròm Ref. 5 . AV Capture level width $=0.0901 \mathrm{eV}$, energy independent $\mathrm{L}=0$ Strength function $\quad=1.8 \mathrm{E}-4$, energy independent $\mathrm{L}=1$ Strength function $\quad=0.272 \mathrm{E}-4$, energy independent $\mathrm{L}=2$ Strength function $=0.0$

Average capture cross section uncetainty at energy $\mathrm{E}$ E (k.eV) Uncertainty (\%) 100

$10 \quad 10$

$4.5 \quad 17$

Resonance integral (capture) 596 b calculation, $590 \pm 10$ b measurement (Ref. 6).

\section{File 3. Neutron Cross Sections}

MT=1. Total Cross Section

The total cross section was evaluated using a least squares spline fit to experimental data for this isotope. Spline fits of experimental data for natural tungsten were also factored into the evaluation (see MAT 1129 for Refs). The total cross section curve was smoothly joined to the evaluated total cross section in the unresolved resonance range below $100 \mathrm{keV}$. Isotopic data references are Whalen ${ }^{7}(10 \mathrm{~J}-650 \mathrm{keV})$, Martin ${ }^{8}(0.65-20 \mathrm{MeV})$, and Foster and Glasgow ${ }^{9}(2.5-15 \mathrm{MeV})$. General references for the total cross section are Goldberg et al. ${ }^{2}$ and Devaney and Foster. 10 The uncertainty in the total cross section is probably less than $7 \%$ over the energy range from $600 \mathrm{keV}$ to $20 \mathrm{MeV}$. Between $300 \mathrm{keV}$ and $600 \mathrm{keV}$ the uncertainty increases to $10 \%$ and from 100 to 200 $\mathrm{keV}$ the uncertainty is estimated to be $15 \%$.

\section{MT $=2$. Elastic Cross Section}

The elastic cross section was obtained by subtracting the nonelastic cross sections from the evaluated total cross section. The elastic cross section is in good agreement with the data of Lister ${ }^{11}$ between $300 \mathrm{keV}$ and $1.5 \mathrm{MeV}$. Between $1 \mathrm{MeV}$ and $9 \mathrm{MeV}$ the evaluated curve lies above 2PLUS-COMNUC results. At $4.3 \mathrm{MeV}$ the cross section is some $15 \%$ lower than the experimental data point of Kinney and Perey ${ }^{12}$ for natural tungsten. Our evaluation is, however, in agreement with their data above $5 \mathrm{MeV}$. 
MT=4. Total Inelastic Cross Section

Equal to the sum of the level excitation and continuun cross sections. The total inelastic cross section for this isotope is in general agreement with the data of owens et al. 13 for natural tungsten between 5 and $7 \mathrm{MeV}$.

$M T=16 . \quad(n, 2 n)$ Cross Section

The semi-empirical techniques of Pearlstein ${ }^{14}$ and $W$. D. Lu et al. ${ }^{15}$ were used to deduce the $n, 2 n$ cross section. The evaluated curve at $14.8 \mathrm{MeV}$ is in agreement with Dilg et $a 1 .^{16}$ and Druzhinin et al. 17

$\mathrm{MT}=17 . \quad(\mathrm{n}, 3 \mathrm{n})$ Cross Section

The $n, 3 n$ cross sections were deduced using the semi-empirical techniques of $S$. Pearlstein ${ }^{14}$ and $W$. D. Lu. ${ }^{15}$ An effective threshold for the $n, 3 n$ reaction was set $1.25 \mathrm{MeV}$ above the theoretical threshold.

$\mathrm{MT}=28$. ( $\mathrm{n}, \mathrm{pn})$ Cross Section

The shape of the $n$,pn excitation curve is based upon the experimental data of $J$. F. Barry et a1. ${ }^{18}$ for ${ }^{186} \mathrm{~W}$ (see MAT 1131). The curve has been shifted in energy by the difference in the reaction $Q$ for this isotope and ${ }^{186} \mathrm{~W}$. The cross section contains contributions from the $n, n p$ and $n, d$ reactions.

$M T=51$. Inelastic Excitation (Direct and Compound Nucleus) to the $2^{+}$Leve1

ihe cross sections were calculated using 2PLUS-COMNUC. They have been renormalized via a least squares fit to data of $D$. Lister et al. ${ }^{19}$ with a $10 \%$ uncertainty in the adjusted curve.

MT=52-58, 91. Inelastic Excitation (Compound Nucleus Only).

Calculation by the COMNUC code for the $329,680,1220,1258,1289$, 1331 , and $1374 \mathrm{keV}$ levels plus the continuum. The 329-keV level excitation curve was renormalized to the data of D. Lister. ${ }^{9}$ The uncertainty in the adjusted cross sections is about $10 \%$.

MT $=102$. Radiative Capture Cross Section

The radiative capture cross section was evaluated between $10 \mathrm{keV}$ and $150 \mathrm{keV}$ using a least squares fit to experimental data (see $M F=2, M T=151$ ). This fit was combined and joined to the theoretical cross sections computed between $100 \mathrm{keV}$ and $4 \mathrm{MeV}$ using the CONNUC code. Above $4 \mathrm{MeV}$ the Comaru results were joined to the collective and direct interaction capture cross sections calculated using the theory of $V$. Benzi and $G$. Reffo. 20 The uncertainty in the radiative capture cross section is probably less than $15 \%$ for energi'zs between $100 \mathrm{keV}$ and $200 \mathrm{keV}$. Above $200 \mathrm{keV}$ the cross 
section is reliable only to the extent of the validity of the theoretical models.

MT $=103 . \quad(n, p)$ Cross Section

The shape of the $n, p$ excitation curve is based upon the experimental data of J. F. Barry et $a 1.18$ for ${ }^{186} \mathrm{~W}$ (see MAT 1131). The curve has been shifted in energy to pass through the data point reported by $M$. Lindner et al. ${ }^{21}(3.5 \pm 0.35 \mathrm{mb}$ at $14.1 \mathrm{MeV})$.

MT=107. $\quad(n, \alpha)$ Cross Section

The $n, \alpha$ cross section evaluation is based upon data of A. Rubino and $D$. Zubke ${ }^{22}$ for ${ }^{181} \mathrm{Ta}$. The few experimental data points for isotopes of tungsten between 14 and $15 \mathrm{HeV}$ qualitatively substantiate the use of this curva. The evaluation has not been extended below $11 \mathrm{MeV}$.

\section{File 4. Neutron Angular Distributions}

MT=2 Elastic Angular Distributions

The angular distributions for elastic scattering are given as Legendre polynomial coefficients in the $\mathrm{cm}$ system. These data were calculated by 2PLUS-COMNUC codes for each isotope. As Iittle isotopic experimental data existed, the calculated data were averaged according to abundance and compared to natural tungsten data. The comparison indicated that the experimental data contained direct inelastic reactions to the first level. With the calculated first-level direct inelastic included, agreement within experimental error was generally indicated over all energies. This agreement also supports the validity of the inelastis angular file below. The natural tungsten experimental data were taken from Refs. 23-30. Error estimates for this section are difficult because it appears that pure elastic scatter has not been measured except at low energies. Because of the good agreement overall with the combined data, this file is estimated to be accurate to $15 \%$ at energies below $1 \mathrm{MeV}$, and within a factor of two at energies above $1 \mathrm{MeV}$ for all scattering angles.

MT $=51-58,91$. Angular Distribution of Inelastic Scattered Neutrons

Angular distributions are given as Legendre polynomial coefficients in the laboratory frame of reference. These data are based upon 2PLUS-COMNUC calculations. They include anisotropic contributions from direct inelastic excitation of the $2^{+}$level. Compound level and continuum excitations are isotropic in the laboratory system. Errors are small simply due to the predominance of isotropy. 


\section{File 5. Neutron Energy Distributions}

$M T=16,17$. Nuclear Temperatures for the $(n, 2 n)$ and $(n, 3 n)$ Reactions

Temperature of first emitted niutron same as for MT=91. Temperatures of second and third emitted neutrons deduced by conservation of energy assuming the kinetic energy of an emitted neutron is $2{ }^{*} \mathrm{KT}$.

MT=91. Nuclear Temperature for Inelastic Continuum

References 31-34 were used in deriving temperatures. Least riuares Weisskopf level spacing was fit to natural tungsten data. Isotopic data adjusted using deformed nucleus level density formula of Gilbert and Cameron. ${ }^{34}$

\section{File 12. Gamma Ray Multiplicities}

MT=102. Multiplicities for Gamma Ray Production from Radiative Capture

Multiplicities at neutron energies below $1 \mathrm{eV}$ are based on a preliminary thermal measurement for natural $\mathrm{W}$ by Jurney ${ }^{35}$ and from $1 \mathrm{eV}$ to $1000 \mathrm{eV}$ on calculations by Yost et al. ${ }^{36}$ At higher energies the multiplicities are based on an analysis of natural $W$ measurements (Dickens, ${ }^{38} E_{n}=1-20 \mathrm{MeV}$ ) using statistical calculations similar to those described by Troubetzkoy, ${ }^{37}$ with the multip? icitles chosen to give rough agreement with the measurements. The theory was used at all energies to divide the natural $W$ data into isotopic components.

File 13. Gamma Ray Production Cross Sections

MT=4. Gamma Ray Production Cross Sections from Inelastic, $(n, 2 n)$, and $(n, 3 n)$ Reactions

The cross sections for discrete photons are based on the level excitation cross sections in $M F=3, M T=51-58$, mainly using the level decay scheme of Way. ${ }^{39}$ The cross sections for continum gamma rays are based on a statistical theory analysis of the Dickens ${ }^{38}$ measurements on natural $\mathrm{W}$. The theory was used for interpolation, smoothing, and separation of the data into isotopic components.

File 14. Gamma Ray Angular Distributions

$\mathrm{MT}=4$, 102. Angular Distributions of Gamma Rays from Radiative Capture, Inelastic, $(n, 2 n)$, and $(n, 3 n)$ Reactions

Gamma rays from all reactions are assumed isotropic in the Jaboratory systern. 


\section{File 15. Gamma Ray Energy Distributions}

MT=4. Energy Distributions of Gamma Rays from Inelastic, $(n, 2 n)$, and $(n, 3 n)$ Reactions

The spectra at all energies are based on a statistical theory analysis of the Dickens ${ }^{38}$ measurements with natural $\mathrm{W}$. The theory was used as described above (MF=13 MT=4). Arbitrary adjustments were made to the theoretical fits in regions where agreement with experiment was poor (mainly below $\mathrm{E}_{\mathfrak{n}}=5 \mathrm{MeV}$ ).

\section{$\mathrm{MT}=102$. Energy Distributions of Gamma Rays From Radiative Capture}

Spectra at neutron energies below $1 \mathrm{eV}$ are based on a preliminary thermal measurement by Jurney ${ }^{35}$ and from $1 \mathrm{eV}$ to $1000 \mathrm{eV}$ on calculations by Yost et $a 1 .^{36}$ At higher energies the spectra are based on statistical calculations using parameters obtained by analyzing the Dickens ${ }^{38}$ measurement for $E_{n}=1-3 \mathrm{MeV}$. The theory was used at all energies to divide the natural $W$ data into separate isotopic components.

(Note: No information is provided in this evaluation on electron production from internal conversion. Such information might be important for local heating and radiation damage problems.)

\section{REFERENCES}

1. A. H. Wapstra and N. B. Gove, Nucl. Data Tables 9, 267 (1971).

2. M. D. Goldberg et al., BNL-325, 2nd Edition, Supplement 2, (1966).

3. Z. M. Bartolome et ai., Nucl. Sci. Eng. 37, p. 137 (1969).

4. F. J. Rahn and H. Camarda, personal comunication, Colimbia (1970).

5. J. L. Cook et al., AAEC/TM-392 (1967).

6. M. Drake, (1968) for American Institute of Phys. Handbook (1972).

7. J. F. Whalen, ANL-7210, 16 (1966).

8. R. C. Martin, PhD Thesis, RPT (1967).

9. D. G. Foster and D. W. Glasgow, Phys. Rev. 3C, 576 (1971).

10. J. J. Devaney and D. G. Foster, Jr., Los Alamos report LA-4928 (1972).

11. D. Lister et al., ANL-7288 (1967).

12. W. E. Kinney and F. G. Perey, ORNL-4803 (1973).

13. R. O. Owens et al., Nucl. Phys. A112, 337 (1968).

14. S. Pearlstein, Nuc1. Sci. Eng. 23, 238 (1965).

15. W. D. Lu et a1., Phys. Rev. C1, 350 (1970).

16. Dilg et a1., Nuc1. Phys. A118, 9 (1968).

17. Druzhinin et a]., Sov. J. Nuc1. Phys. 4, 515 (1966).

18. J. F. Barry et al., Proc. Phys. Soc. $7 \overline{4}, 632$ (1959).

19. D. Lister et al., Phys. Rev. 162, 1077 (1967).

20. V. Benzj, and G. Reffo, CCDN-NW10, ENEA Neutron Compilation Center (1969).

21. M. Lindner et a1., Lawrence Livermore Laboratory, private communication (1972).

22. A. Rubino and D. Zubke, Nucl. Phys. 85, 606 (1966).

23. A. B. Smith and P. A. Moldauer, BAPS $\underline{5}, 409$ (1960).

24. F. T. Kuchni: et al., Phys. Rev. 176, 1405 (1968). 
25. W. Walt and H. Barscha1l, Phys. Rev. 93, 1062 (1954).

26. K. Tsukada et a1., Nucl. Phys. A125, 641 (1966).

27. R. W. Hill, Phys. Rev. 109,2105 (1958).

28. W. Kinney, private communication (1972).

29. D. B. Thomson, Phys. Rev. 129, 1649 (1963).

30. H. Nauta, Nucl. Phys. 2, $1 \overline{24}$ (1956).

31. S. G. Buccino et al, Nuc1. Phys. 60, 17 (1964).

32. R. O. Owens and J. H. Towle, Nuc1. Phys. A112, 337 (1968).

33. W. E. Kinney, ORNL, private communication (1972).

34. A. Gilbert and A. G. W. Cameron, Can. J. Phys. 43, 1446 (1965).

35. E. T. Jurney, private communication (1973).

36. K. J. Yost, J. E. WhIte, C. Y. Fu, and W. E. Ford, Nucl. Sci. Eng. 47, 209 (1972).

37. E. S. Troubetzkoy, Phys, Rev. 122, 212 (1961).

38. J. K. Dickens, private communication (1972).

39. K. Way, Nuclear Data B1-1, 1 (1966). 
SUMMARY DOCUNENTATION FOR ${ }^{183} \mathrm{~W}$

\author{
by \\ P. G. Young, J. Otter, ${ }^{\dagger}$ E. Ottewitte, ${ }^{\dagger}$ and P. Rose \\ Los Alamos Scientific Labcratory \\ Los Alamos, New Mexico
}

\title{
I. SUMMARY
}

The ${ }^{283} \mathrm{~W}$ evaluation for $\mathrm{ENDF} / \mathrm{B}-\mathrm{V}$ (MAT 1129) was carried over intact from Version IV with only minor format changes being made. The evaluation of the neutron files was performed at Atonics International and is documented in TI-707-130-026 (1973). The gamma-ray production data were evaluated at Los Alamos Scientific Laboratory and are documented in LA-5793 (1975). The ENDF/B-V data span the energy range $10^{-5} \mathrm{eV}$ to $20 \mathrm{MeV}$.

\section{ENDF/B-V FILES}

File 1. General Information

$$
\text { MT }=451 \text {. Descriptive Data }
$$

Atomic mass and Q-values taken from Ref. 1.

File 2. Resonance Parameters

$$
\begin{aligned}
& \text { MT=151. (A) Resolved Resonances Evaluation } \\
& \text { Potential scattering cross section }=8.0 \pm 1.0 \mathrm{~b} \text { at } E_{n}=0 \text {. } \\
& 2200 \mathrm{~m} / \mathrm{s} \text { Cross Sections (barns) } \\
& \text { CALC } \quad \text { MEAS (Ref. 2) } \\
& \text { CAP } \overline{10.0} \quad \overline{10.2 \pm 3.0} \\
& \text { SCAT } \quad 3.4 \\
& \text { Resolved Resonance Parameters } \\
& -1.5 \mathrm{ev} \text { from fit to capture } \\
& 0-150 \mathrm{eV} \text { from Ref. } 2 \\
& 150-175 \mathrm{eV} \text { from evaluation, Refs. } 2 \text { and } 3 \\
& 175 \text { - } 760 \mathrm{eV} \text { from Ref. } 3
\end{aligned}
$$

$\bar{†}_{\text {Atomics International }}$ 
MT=151. (B) Unresolved Resonances Evaluation

Potential scattering cross section $=8.5 \pm 1.0 \mathrm{~b}$ at $E_{n}=0$.

Total cross section $=11.3 \mathrm{~b}$ at $\mathrm{E}_{\mathrm{n}}=45 \mathrm{k \in V}$ (calculated).

Unresolved resonance parameters from automated optimized fit to the evaluated measured capture cross section. The competitive reaction width for $L=2, J=1$ is for the $L$ to $L-2$ elastic scattering process.

AV $L=0$ level spacing $\left(E_{n}=0\right)=12.53 \mathrm{eV}$, energy dep. from Ref. 4 .

AV Capture level width $\quad=0.0801 \mathrm{eV}$, energy independent

$\mathrm{L}=0$ Strength function $\quad=2.125 \mathrm{E}-4 \mathrm{eV}$, energy independent

$\mathrm{L}=1$ Strength function $\quad=0.227 \mathrm{E}-4 \mathrm{eV}$, energy independent

$\mathrm{L}=2$ strength function $\quad=2.56 \mathrm{E}-4 \mathrm{eV}$, energy independent

Average capture cross section uncertainty at energy $\mathrm{E}$

E (keV) Uncertainty (\%)

$45 \quad 11$

$10 \quad 12$

520

$2.5 \quad 40$

Resonance integral (capture) 355 b calculation, $380 \pm 15$ b measurement (Ref. 5).

File 3. Neutron Cross Sections

$M T=1$. Total Cross Section

There are no experimental data for ${ }^{183} \mathrm{~W}$. The total cross section was evaluated using a least squares spline fit to experimental data for natural tungsten. ${ }^{6-10}$ The total cross section curve was smoothly joined to the evaluated total cross section in the energy range below $100 \mathrm{keV}$. The lack of direct experimental data introduces a large uncertainty in the total cross section between $100 \mathrm{keV}$ and $700 \mathrm{keV}$ (see Ref. 10).

MT $=2$. Elastic Cross Section

The elastic cross section was obtained by subtracting the nonelastic cross sections from the evaluated total cross section. Between 1 $\mathrm{MieV}$ and $9 \mathrm{MeV}$, the evaluated curve lies above 2PLUS-COMNUC results. At $4.3 \mathrm{MeV}$ the cross section is sone 15\% lower than the experimental data point of Kinney and Perey ${ }^{1}$ for natural tungsten. our evaluation 1s, however, in agreement with their data above $5 \mathrm{MeV}$.

MT=4. Total Inelastic Cross Section

Equrl to the sum of the level excitation and continuum cross rctilons. The total inelastic cross section for this isotope is in general agreenent with the data of Owens et al. ${ }^{12}$ for natural tungsten between 5 and $7 \mathrm{MeV}$. 
$\mathbb{M T}=16 . \quad(n, 2 n)$ Cross Section

The semi-empirical techniques of Pearlstein ${ }^{13}$ and $W . D$. Lu et a1.14 were used to deduce the $n, 2 n$ cross section.

$M T=17 . \quad(n, 3 n)$ Cross Section

The $n, 3 n$ cross sections were deduced using the semi-empirical techniques of S. Pearlstein ${ }^{13}$ and W. D. Lu. ${ }^{14}$ An effective threshold for the $n, 3 n$ reaction was set $1.25 \mathrm{MeV}$ above the theoretical threshold.

$\mathrm{MT}=28 . \quad(\mathrm{n}, \mathrm{pn})$ Cross Section

The shape of the $n, p n$ excitation curve is based upon the experimental data of J. F. Barry et al. ${ }^{15}$ for ${ }^{186} \mathrm{~W}$ (see MAT 1131). The curre has been shifted in energy by the difference in the reaction $Q$ for this isotope and ${ }^{186} \mathrm{~W}$. The cross section contains contributions from the $n, n p$ and $n, d$ reactions.

$\mathrm{MT}=51$, 52. Inelastic Excitation (Direct and Compound Nucleus) to the 46.5 and $99 \mathrm{keV}$ Levels.

The cross sections were calculated using the 2PLUS-COMNUC codes. No experimental data has been reported.

MT=53-59, 91. Inelastic Excitation (Compound Nucleus Only).

Calculation by the COMNCC sode for the 207, 209, 292, 309, 412, 453, and 595-keV levels plus the continuum. The theoretical cross sections were used without modification.

MT=102. Radiative Capture Cross Section

The radiative capture cross section was evaluated between $10 \mathrm{keV}$ and $100 \mathrm{keV}$ using a least squares fit to experimental data (see $M F=2, M T=151$ ). This fit was combined and joined to the theoretical cross sections computed between $100 \mathrm{keV}$ and $4 \mathrm{MeV}$ using the COMNUC code. Above $4 \mathrm{MeV}$ the COMNUC results were joined to the collective and direct interaction capture cross sections calculated using the theory of $V$. Benzi and G. Reffo. ${ }^{1 E}$ The uncertainty in the radiative capture cross section is $11 \%$ between 45 $\mathrm{keV}$ and $90 \mathrm{keV}$. Between $90 \mathrm{keV}$ and $200 \mathrm{keV}$ the uncertainty is probably less than 25\%. Above $200 \mathrm{keV}$ the cross section is reliable only to the extent of the validity of the theoretica? models,

MT=103. (n,p) Cross Section

The shape of the $n, p$ excitation curve is based upon the experimental data of J. F. Barry et al. ${ }^{15}$ for ${ }^{186} \mathrm{~W}$ (see MAT 1131). The curve has been shifted in energy to pass through the data point reported by M. Lindner et $\mathrm{al}^{17}(4.2 \pm 0.42 \mathrm{mb}$ at $14.1 \mathrm{MeV})$. 
MT=107. $(n, \alpha)$ Cross Section

The $n, \alpha$ cross section evaluation is based upon data of $A$. Rubino and $D$. Zubke ${ }^{18}$ for ${ }^{181} \mathrm{Ta}$. The few experimental data points for isotopes of tungsten between 14 and $15 \mathrm{MeV}$ qualitatively substantiate the use of this curve. The evaluation has not been extended below $11 \mathrm{MeV}$.

File 4. Neutron Angular Distributions

MT=2, Elastic Angular Distributions

The angular distributions for elastic scattering are given as Legendre polynomial coefficients in the cm system. These data were calculated by 2PLUS-COMNUC codes for each isotope. As little isotopic experimental data existed, the calculated data were averaged according to abundance and compared to natural tungsten data. The comparison indicated that the experimental data contained direct inelastic reactions to the first 2 levels. With the calculated first-and second-level direct inelastic included, agreement within experimental error was generally iildicated over all energies. This agreement also supports the validity of the inelastic angular file below. The natural tungsten experimental data were taken from Refs. 19-26. Error estimates for this section are difficult because it appears that pure elastic scatter has not been measured except at low energies. Because of. the good agreement overall with the combined data, this file is estimated to be accurate to $15 \%$ at energies below $1 \mathrm{MeV}$, and within a factor of two at energies above $1 \mathrm{MeV}$ for all scattering angles.

MT=51-59, 91. Angular Distribution of Inelastic Scattered Neutrons

Angular distributions are given as Legendre polynomial coefficients in the laboratory frame of reference. These data are based upon 2PLUS-COMNUC calculations. They include anisotropic contributions from direct inelastic excitation of the $3 / 2^{-}$and $5 / 2^{-}$ levels. Compound level and continum excitations are isotropic in the laboratory system. Errors are small simply due to the predominance of isotropy.

\section{File 5. Neutron Energy Distributions}

$\mathrm{MT}=16,17$. Nuclear Temperatures for the $(n, 2 n)$ and $(n, 3 n)$ Reactions

Temperature of first emitted neutron same as for $M T=91$. Temperatures of second and third emitted neutrons deduced by conse:vation of energy assuming the kinetic energy of an emitted neutron is $2 * \mathrm{KT}$.

MT=91. Nuclear Temperatures For Inelastic Continuum

References 27-30 were used in deriving temperatures. Least squares Weisskopf level spacing was fit to natural tungsten data. 
Isotopic data adjusted using deformed nucleus level density formula of Gilbert and Cameron. 30

File 12. Gamma Ray Multiplicities

MT=102. Multiplicities for Gamma Ray Production from Radiative Capture

Multiplicities at neutron energies below $1 \mathrm{eV}$ are based on a preliminary thermal measurement for natural $\mathrm{V}$ by Jurney ${ }^{31}$ and from $1 \mathrm{eV}$ to $1000 \mathrm{eV}$ on calculations by Yost et al. ${ }^{32}$ At higher ene=gles the multiplicities are based on an analysis of natural measurements (Dickens, ${ }^{34} \mathrm{E}_{\mathbf{n}}=1-20 \mathrm{MeV}$ ) using statistical calculations similar to those described by Troubetzkoy, ${ }^{3}$ with the multiplicities chosen to give rough agreement with the measurements. The theory was used at ali energies to divide the natural $W$ data into isotopic components.

\section{File 13. Gamma Ray Production Cross Sections}

MT=4. Gamna Ray Production Cross Sections from Inelastic, $(n, 2 n)$, and $(n, 3 n)$ Reactions

The cross sections for discrete photons are irased on the level excitation cross sections in $M F=3, M T=51-59$, mainly using the level decay scheme of Artna. ${ }^{35}$ The cross sections for continuum gamma rays are based on a statistical theory analysis of the Dickens ${ }^{34}$ measurements on natural $W$. The theory was used for interpolation, smoothing, and separation of the data into isotopic components.

\section{File 14. Gamma Ray Angular Distributions}

$M T=4,102$. Angular Distributions of Gamma Rays from Radiative Capture, Inelastic, $(n, 2 n)$, and $(n, 3 n)$ Reactions

Gamma rays from all reactions are assumed isorropic in the laboratory system.

File 15. Gamma Ray Energy Distributions

$M T=4$. Energy Distributions of Gamma Rays from Inelastic, $(n, 2 n)$, and $(n, 3 n)$ Reactions

The spectra at all energies are based on a statistical theory analysis of the Dickens ${ }^{34}$ measurements with natural $\mathrm{W}$. The theory was used as described above $(M F=13, M T=4)$. Arbitrary adjustments were made to the theoretical fits in regions where agreement with experiment was poor (mainly below $\mathrm{E}_{\mathrm{n}}=5 \mathrm{MeV}$ ).

\section{$\mathrm{MT}=102$. Energy Distributions of Gamma Rays from Radiative Capture}

Spectra at neutron energies below $1 \mathrm{eV}$ are based on a preliminary thermal measurement by Jurney ${ }^{31}$ and from $1 \mathrm{eV}$ to $1000 \mathrm{eV}$ on 
calculations by Yost et al. ${ }^{32}$ At higher energies the spectra are based on statistical calculations using parameters obtained by analyzing the Dickens ${ }^{34}$ measurement for $E_{n}=1-3 \mathrm{MeV}$. The theory was used at all energies to divide the natural $\mathrm{W}$ data into separate Isotopic components.

(Note: No information is provided in this evaluation on electron production from internal conversion. Such information might be important for local heating and radiation damage problems.)

\section{REFERENCES}

1. A. H. Wapstra and N. B. Gove, Nucl. Data Tables 9 , 267 (1971).

2. M. D. Goldberg et al, BNL-325, 2nd Edition, Supplement 2 (1966).

3. Z. M. Bartolume et al., Nucl. Sci. Eng. 37, p. 137 (1969).

4. J. L. Cook et al., AAEC/TM-392 (1967).

5. M. Drake, (1968) for American Institute of Phys. Handbook (1972).

6. J. F. Whalen, ANL-7210, 16 (1966).

7. M. Davadeenam, PhD Thesis Duke University (1967).

8. W. H. Kao et al., Chinese J. Phys. 5, 43 (1967).

9. J. M. Peterson et al., Phys. Rev. 120,521 (1960).

10. J. J. Devaney and D. G. Foster, Jr., Los Alamos report LA-4928 (1972).

11. W. E. Kinney and F. G. Perey, ORNL-4803 (1973).

12. R. O. Owens et al., Nucl. Phys. A112, 337 (1968).

13. S. Pearlstein, Nucl. Sci. Eng. 23,238 (1965).

14. W. D. Lu et a1., Phys. Rev. C1, 350 (1970).

15. J. F. Barry et al., Proc. Phys. Soc. 74, 632 (1959).

16. V. Benzi and G. Reffo, CCDN-NW10, ENEA Neutron Compilation Center (1969).

17. M. Lindner et al., Lawrence Livermore Laboratory, private communication (1972).

18. A. Rubino and D. Dubke, Nucl. Phys. 85, 606 (1966).

19. A. B. Smith and P. A. Moldauer, BAPS 509 (1960).

20. F. T. Kuchnir et al., Phys. Rev. 176, 1405 (1968).

21. W. Walt and H. Barschall, Phys. Rev. 93, 1062 (1954).

22. K. Tsukada et al., Nucl. Phys. A125, 641 (1966).

23. R. W. Hill, Phys. Rev. 109, 2105 (1958).

24. W. Kinney, private communication (1972).

25. D. B. Thomson, Phys. Rev. 129, 1649 (1953).

26. H. Nauta, Nuc1. Phys. 2,124 (1956).

27. S. G. Buccino et al., Nucl. Phys. 60, 17 (1964).

28. R. O. Owens and J. H. Towle, Nucl. Phys. A112, 337 (1968).

29. W. E. Kinney, ORNL, private communication (1972).

30. A. Gilbert and A. G. W. Cameron, Can. J. Phys. 43, 1446 (1965).

31. E. T. Jurney, private communication (1973).

32. K. J. Yost, J. E. White, C. Y. Fu, and W. E. Ford, Nucl. Sci. Eng. 47, 209 (1972).

33. E. S. Troubetzkoy, Phys. Rev 122, 212 (1961).

34. J. K. Dickens, private communication (1972).

35. A. Artna, Nuclear Data B1-1, 37 (1966). 
SUMMARY DOCUMENTATION FOR ${ }^{184} \mathrm{~W}$

\author{
by \\ P. G. Young, J. Otter, ${ }^{\dagger}$ E. Ottewitte, ${ }^{\dagger}$ and P. Rose ${ }^{\dagger}$ \\ Los Alamos Scientific Laboratory \\ Los Alamos, New Mexico
}

\title{
I. SUMMARY
}

The ${ }^{184} \mathrm{~W}$ evaluation for ENDF/B-V (MAT 1130) was carried over intact from Version IV with only minor format changes being made. The evaluation of the neutron files was performed at Atomics International and is documented in TI-707-130-026 (1973). The gamma-ray production data were evaluated at Los Alamos Scientific Laboratory and are documented in LA-5793 (1975). The ENDF/B-V data span the energy range $10^{-5} \mathrm{eV}$ to $20 \mathrm{MeV}$.

II. ENDF/B-V FILES

File 1. General Information

MT $=451$. Descriptive Data

Atomic mass and Q-values taken from Ref. 1 .

File 2. Resonance Parameters

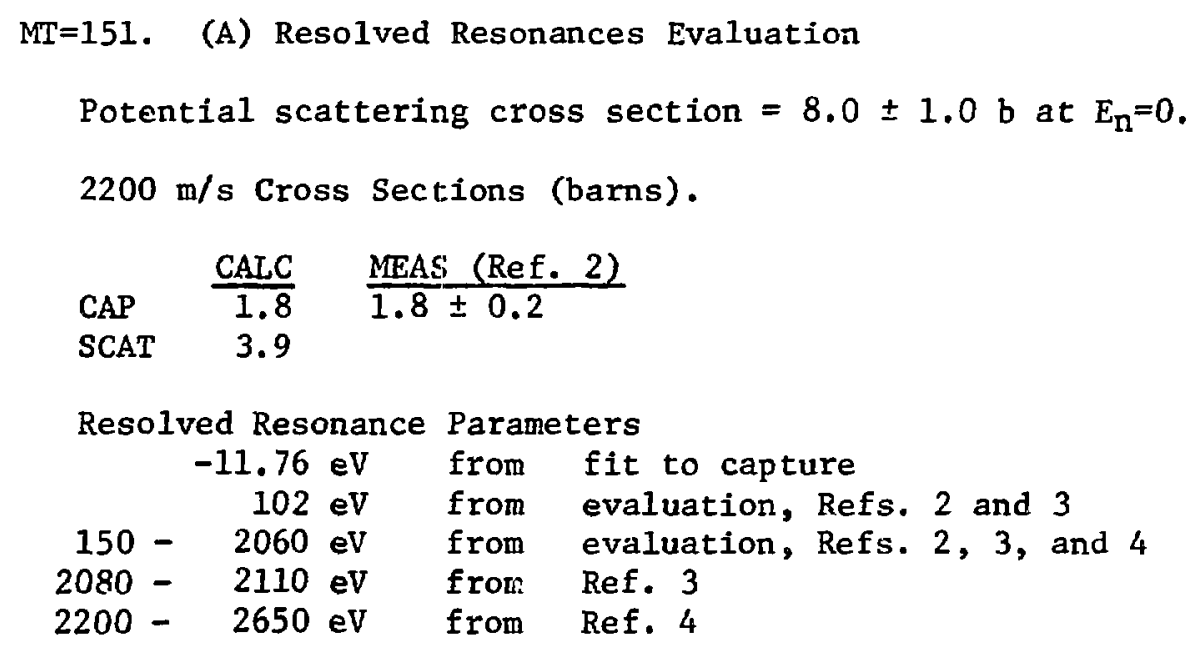

$\bar{\uparrow}_{\text {Atomics International }}$ 
$M T=151 . \quad$ (B) Unresolved Resonances Evaluation

Potential scattering cross section $=8.5 \pm 1.0$ b at $E_{n}=0$. Total cross section $=\ldots 1 \mathrm{~b}$ at $\mathrm{E}_{\mathrm{n}}=100 \mathrm{keV}$ (calculated).

Unresolved resonance parameters from automated optimized fit to the evaluated measured capture cross section.

AV $L=0$ level spacing $\left(E_{n}=0\right)=80.3 \mathrm{eV}$, energy dep. from Ref. 5 .

AV Capture level width $\quad=0.0731 \mathrm{eV}$, energy indepencent

$\mathrm{L}=0$ Strength function $\quad=2.3 \mathrm{E}-4$, energy independent

$\mathrm{L}=1$ Strength function $\quad=0.18 \mathrm{E}-4$, energy independent

$\mathrm{L}=2$ Strength function $\quad=1.45 \mathrm{E}-4$, energy independent

Average capture cross section uncertainty at energy $E$

$\mathrm{E}$ (keV) Uncertainty (\%)

$\begin{array}{rr}100 & 15 \\ 90 & 10 \\ 10 & 10 \\ 4.5 & 20\end{array}$

Resonance integral (capture) $16.2 \mathrm{~b}$ calculation, $13 \pm 2 \mathrm{~b}$ measurement (Ref, 6).

File 3. Neutron Cross Sections

MT $=1 . \quad$ Total Cross Section

The total cross section was evaluated using a least squares spline fit to experimental data for this isotope. Spline fits of experimental data for natural tungsten were also factored into the evaluation (see MAT 1129 for references). The total cross section curve was smoothly joined to the evaluated total cross section in the unresolved resonance range below $100 \mathrm{keV}$. Isotopic data references are Whalen ${ }^{7}(100-650 \mathrm{keV})$, Martin ${ }^{8}(0.65-20 \mathrm{MeV})$, and Foster and Glasgow ${ }^{9}(2.5-15 \mathrm{MeV})$. General references for the total cross section are Goldberg et al. ${ }^{2}$ and Devaney and Foster. 10 The uncertainty in the total cross section is probably less than $7 \%$ over the energy range from $600 \mathrm{keV}$ to $20 \mathrm{MeV}$. Between $300 \mathrm{keV}$ and $600 \mathrm{keV}$ the uncertainty increases to $10 \%$ and from 100 to 200 $\mathrm{keV}$ the uncertainty is estimated to be $15 \%$.

$\mathrm{MT}=2$. Elastic Cross Section

The elastic cross section was obtained by subtracting the nonelastic cross sections from the evaluated total cross section. The elastic cross section is in good agreement with the data of Lister 1 between $300 \mathrm{keV}$ and $0.8 \mathrm{MeV}$. Between $0.8 \mathrm{MeV}$ and $1.5 \mathrm{MeV}$, the elastic crosis section is somewhat higher than the experimenta1 data. Between $1 \mathrm{MeV}$ and $9 \mathrm{MeV}$ the evaluation curve lies above 2PLUS-COMNUC results. At $4.3 \mathrm{MeV}$ the cross section is some $15 \%$ lower than the experimental data point of Kinney and Perey ${ }^{12}$ for natural tungsten. Our evaluation is, however, in agreement with their data above $5 \mathrm{MeV}$. 
MT=4. Total Inelastic Cross Section

Equal to the sum of the level excjtation and continuum cross sections. The total inelastic cross section for this isotope is in general agreement with the data of. Owens et a1. ${ }^{3}$ for natural tungsten between 5 and $7 \mathrm{MeV}$.

$\mathrm{MT}=16 . \quad(n, 2 n)$ Cross Section

The semi-empirical techniques of Pearlstein ${ }^{14}$ and W. D. Lu et a1.15 were used to deduce the $n, 2 n$ cross section.

$M T=17 . \quad(n, 3 n)$ Cross Section

The $n, 3 n$ cross sections were deduced using the semi-empirical techniques of S. Pearlstein ${ }^{14}$ and W. D. Lu. ${ }^{15}$ An effective threshold for the $n, 3 n$ reaction was set $1.25 \mathrm{MeV}$ above the theoretical threshold.

MT=28. (n,pn) Cross Section

The shape of the $n, p n$ excitation curve is based upon the experimental data of $J$. F. Barry et $a 1 .{ }^{16}$ for ${ }^{18 \varepsilon_{W}}$ (see MAT 1131). The curve has been shifted in energy by the difference in the reaction $Q$ for this isotope and ${ }^{186} \mathrm{~W}$. The cross section contains contributions from the $n, n p$ and $n, d$ reactions.

$M T=51$. Inelastic Excitation (Direct and Compound Nucleus) to the $2^{+}$Leve1

The cross sections were calculated using 2PLUS-COMNUC. They have been renormalized via a least squares fit to data of $D$. Lister et al. 17 with a $10 \%$ uncertainty in the adjusted curve.

MT=52-59, 91. Inelastic Excitation (Compound Nucleus Only).

Calculation by the COMNUC cone for the 365, 748, 904, 1007, 1135, 1223,1270 , and $1287 \mathrm{keV}-1$ evels plus the continuum. The 365-, 904-, and 1007-keV level excitation curves were renormalized to the data of $\mathrm{D}$. Lister. ${ }^{17}$ The uncertainty is about $10 \%$ in the $365-\mathrm{keV}$ level data and $15 \%$ in the $904-$ and $1007-\mathrm{keV}$ level data.

MT=102. Radiative Capture Cross Section

The radiative capture cross section was evaluated between $10 \mathrm{keV}$ and $150 \mathrm{keV}$ using a least squares fit to experimental data (see $M F=2, M T=151$ ). This fit was combined and joined to the theoretical cross sections computed between $100 \mathrm{keV}$ and $4 \mathrm{MeV}$ using the COMNUC code. Above $4 \mathrm{MeV}$ the COMaUC results were joined to the collective and direct interaction capture cross sections calculated using the theory of $\mathrm{V}$. Benzi and $G$. Reffo. ${ }^{18}$ The uncertainty in the radiative capture cross section is probably less than $15 \%$ for energies between $100 \mathrm{keV}$ and $200 \mathrm{keV}$. Above $200 \mathrm{keV}$ the cross 
section is reliable only to the extent of the validity of the theoretical models.

$M T=103 . \quad(n, p)$ Cross Section

The shape of the $n, p$ excitation curve is based upon the experimental data of J. F. Barry et $21 .^{16}$ for ${ }^{186} \mathrm{~W}$ (see MAT 1131). The curve has been shifted in energy to agree with the ratio of $(n, p)$ measurements reported by Coleman ${ }^{19}$ for ${ }^{184} \mathrm{~W}$ and ${ }^{186} \mathrm{~W}$ at $14.5 \mathrm{HeV}$.

MT $=107 . \quad(n, \alpha)$ Cross Section

The $n, \alpha$ cross section evaluation is based upon data of A. Rubino and $D$. Zubke ${ }^{20}$ for ${ }^{181} \mathrm{Ta}$. The few experimental data points for isotopes of tungsten between 14 and $15 \mathrm{MeV}$ qualitatively substantiate the use of this curve. The evaluation has not been extended below $11 \mathrm{MeV}$.

\section{Fyle 4. Neutron Angular Distributions}

\section{$\mathrm{MT}=2$. Elastic Angular Distributions}

The angular distributions for elastic scatterirg are given as Legendre polynomial coefficients in the $\mathrm{cm}$ system. These data were calculated by 2 PLUS-COMNUC codes for each isotope. As little isotopic experimental data existed, the calculated data were averaged according to abundance and compared to natural tungsten data. The comparison indicated that the experimental data contained direct inelastic reactions to the first level. With the calculated first-level direct inelastic included, agreement within experimental error was generally indicated over all energies. This agreement also supports the validity of the inelastic angular file below. The natural tungsten experimental data were taken from Refs. 21-28. Error estimates for this section are difficult because it appears that pure elastic scatter has not been measured except at low energies. Because of the good agreement overall with the combined data, this file is estimated to be accurate to $15 \%$ at energies below $1 \mathrm{MeV}$, and within a factor of two at energies above $1 \mathrm{MeV}$ for all scattering angles.

MT $=51-59$, 91. Angular Distribution of Inelastic Scattered Neutrons

Angular distributions are given as Legendre polynomial coefficients in the laboratory frame of reference. These data are based upon 2PLUS-COMNUC calculations. They include anisotropic contributions from direct inelastic excitation of the $2^{+}$level. Compound level and continuum excitations are isotropic in the $1 \mathrm{ab}-$ oratory system. Errcrs are small simply due to the predoninance of isotropy. 
File 5. Neutron Energy Distributions

MT $=16,17$. Nuclear Temperatures for the $(n, 2 n)$ and $(n, 3 n)$ Reactions

Temperature of first emitted neutron same as for MT $=91$. Temperatures of second and third emitted neutrons deduced by conservation of energy assuming the kinetic energy of an emitted neutron is $2 * \mathrm{KT}$.

$\mathbb{M T}=91 . \quad$ Nuclear Temperature for Inelastic Continuum

References 29-32 were used in deriving temperatures. Least squares Weisskopf level spacing was fit to natural tungsten data. Isotopic data adjusted using deformed nucleus level density formula of Gilbert and Cameron. ${ }^{32}$

File 12. Gamma Ray Multiplicities

MT=102. Multiplicities for Gamma Ray Production from Radiative Capture

Multiplicities at neutron energies below $1 \mathrm{eV}$ are based on a preliminary thermal measurement for natural $\mathrm{W}$ by Jurney ${ }^{3}$ and from $1 \mathrm{eV}$ to $1000 \mathrm{eV}$ on calculations by Yost et al. ${ }^{34}$. At higher energies the multiplicities are based on an analysis of natural $\mathrm{W}$ measurements (Dickens, ${ }^{36} E_{n}=1-20 \mathrm{MeV}$ ) using statistical calculations similar to those described by Troubetzkoy, ${ }^{35}$ with the multiplicities chosen to give rough agreement with the measurements. The theory was used at all energies to divide the natural $\mathrm{W}$ data inะo isotopic components.

\section{File 13. Ganma Ray Production Cross Sections}

$M T=4$. Gamma Ray Production Cross Sections from Inelastic, $(n, 2 n)$, and $(n, 3 n)$ Reactions

The cross sections for discrete photons are based on the level excitation cross sections in $M F=3, M T=51-59$, mainly using the level decay scheme of Martin. ${ }^{37}$ The cross sections for continuum gamma rays are based on a statistical theory analysis of the Dickens ${ }^{36}$ measurements on natural $\mathrm{W}$. The theory was used for interpolation, smoothing, and separation of the data into isotopic components.

File 14. Gamma Ray Angular Distributions

$M=4,102$. Angular Distributions of Gamma Rays from Radiative Capture, Inelastic, $(n, 2 n)$, and $(n, 3 n)$ Reactions

Gamma rays from all reactions are assumed isotropic in the laboratory system. 


\section{File 15. Gamma Ray Energy Distributions}

$M T=4$. Energy Distributions of Gama Rays from Inelastic, $(n, 2 n)$, and $(n, 3 n)$ Reactions

The spectra at all energies are based on a statistical theory analysis of the Dickens ${ }^{36}$ preasurements with natural $W$. The theory was used as described above ( $M F=13, M T=4)$. Arbitrary adjustments were made to the theoretical fits in regions where agreement with experiment was poor (mainly below $E_{n}=5 \mathrm{MeV}$ ).

\section{Mr: $=102$. Energy Distributions of Gamma Rays from Radiative Capture}

Spectra at neutron energies below $1 \mathrm{eV}$ are based on a preliminary thermal measurement by Jumey ${ }^{33}$ and from $1 \mathrm{eV}$ to $1000 \mathrm{eV}$ on calculations by Yost et $a 1 .^{34}$ At higher energies the spectra are based on statistical calculations using parameters obtained by analyzing the Dickens ${ }^{36}$ measurement for $E_{\mathrm{n}}=1-3 \mathrm{MeV}$. The theory was used at all energies to divide the natural $W$ data into separate isotopic components.

(Note: No information is provided in this evaluation on electron production from internal conversion. Such information might be important for local heating and radiacion damage problems).

\section{REFERENCES}

1. A. H. Wapstra and N. B. Gove, Nucl. Data Tables 9,267 (1971).

2. M. D. Goldberg et al., BNL-325, 2nd Edition, Supplement 2, (1966).

3. Z. M. Bartolome et al., Nuc1. Sci. Eng. 37, p. 137 (1969).

4. F. J. Rahn and H. Camarda, personal communication, Columbia (1970).

5. J. I. Cook et al., AAEC/TM-392 (1967).

6. M. Drake, (1968) for American Institute of Phys. Handbook (1972).

7. J. F. Whalen, ANL-7210, 16 (1966).

8. R. C. Martin, PhD Thesis, RPT (1967).

9. D. G. Foster, and D. W. Glasgow, Phys. Rev. 3C, 576 (1971).

10. J. J. Devaney and D. G. Foster, Jr., Los Alamos report LA-4928 (1972).

11. D. Lister et al., ANL-7288 (1967).

12. W. E. Kinney and F. G. Perey, ORNL-4803 (1973).

13. R. 0. Owens et al., Nucl. Phys. A112, 337 (1968).

14. S. Pearlstein, Nucl. Sci. Eng. 23, 238 (1965).

15. W. D. Lu et al., Phys. Rev. C1, 350 (1970).

16. J. F. Barry et al., Proc. Phys. Soc. 74, 632 (1959).

17. D. Iister et a1., Phys. Rev. 162, $107 \overline{7}$ (1967).

18. V. Benzi and G. Reffo, CCDN-NW10, ENEA Neutron Compilation Center (1969).

19. R. F. Coleman, Proc. Phys. Soc. 73, 215 (1959).

20. A. Rubino and D. Zubke, Nucl. Phys. 85, 606 (1966).

21. A. B. Smith and P. A. Moldauer, BAPS $\underline{5}, 409$ (1960).

22. F. T. Kuchnir et a1., Phys. Rev. 176, 1405 (1968).

23. W. Walt and H. Barschal1, Phys. Rev. 93, 1062 (1954).

2:. K. Tsukada et a1., Nucl. Phys. A125, 641 (1966).

25. R. W. Hill, Phys. Rev. 109, 2105 (1958). 
26. W. Kinney, private communication (1972).

27. D. B. Thomson, Phys. Rev. 129, 1649 (1963).

28. H. Nauta, Nucl. Phys. 2, 124 (1956).

29. S. G. Buccino et al., Nucl. Phys. 60, 17 (1964).

30. R. O. Owens and J. H. Towle, Nucl. Phys. Al12, 337 (1958).

31. W. E. Kinney, ORNL, private communication (1972).

32. A. Eilbert and A. G. W. Cameron, Can. J. Phys. 43, 1446 (1965).

33. E. T. Jurney, private communication (1973).

34. K. J. Yost, J. E. White, C. Y. Fu, and W. E. Ford, Nucl. Sci. Eng. 47, 209 (1972).

35. E. S. Troubetzkoy, Phys. Rev. 122, 212 (1961).

36. J. K. Dickens, private communication (1972).

37. M. J. Martin, Nuclear Data B1-1, 63 (1966). 
SUMMARY DOCUMENTATION FOR ${ }^{186}$ W

\author{
by \\ P. G. Young, J. Otter, ${ }^{\dagger}$ E. Ottewitte, ${ }^{\dagger}$ and P. Rose ${ }^{\dagger}$ \\ Los Alamos Scientific Laboratory \\ Los Alamos, New Mexico
}

I. SUMMARY

The ${ }^{186} \mathrm{~W}$ evaluation for ENDF/B-V (MAT 1131) was carried over intact from Version IV with only minor format changes being made. The evaluation of the neutron files was performed at Atomics International and is documented in TI-707-130-026 (1973). The gamma-ray production data ware evaluated at Los Alamos Scientific Laboratory and are documented in LA-5793 (1975). The ENDF/ $B-V$ data span the energy range $10^{-5}$ eV to $20 \mathrm{MeV}$.

II. ENDF/B-V FILES

File 1. General Information

MT=451. Descriptive Data

Atomic mass and Q-values taken from Ref. 1 .

File 2. Resonance Parameters

MT=151. (A) Resolved Resonances Evaluation

Potential scattering cross section $=8.0 \pm 1.0 \mathrm{~b}$ at $\mathrm{E}_{\mathrm{n}}=0$.

$2200 \mathrm{~m} / \mathrm{s}$ Cross Sections (barns).

$\begin{array}{lcc} & \text { CALC } & \text { MEAS (Ref.2) } \\ \text { CAP } & 37.5 & 38 \pm 2 \\ \text { SCAT } & 0.39 & \end{array}$

Resolved Resonance Parameters

$18.81 \mathrm{eV}$ from evaluation of Refs. 2 and 4 plus adjustment from fit to capture cross

$100-250 \mathrm{eV}$ from evaluation of Refs. 2-4

$250-3200 \mathrm{eV}$ from evaluation of Refs. 3-4

†tomics International 


\section{MT=51. (B) Unresolved Resonances Evaluation}

Potential scattering cross section $=8.5 \pm 1.0 \mathrm{~b}$ at $\Sigma_{\mathrm{n}}=0$. Total cross section $=9.1 \mathrm{~b}$ at $\mathrm{E}_{\mathrm{n}}=100 \mathrm{keV}$ (calculated).

Unresolved resonance parameters from automated optimized fit to the evaluated measured capture cross section.

AV $L=0$ level spacing $(E=0)=99.1 \mathrm{eV}$, energy dep. from Ref. 5

AV Capture level width $\quad=0.0530 \mathrm{eV}$, energy independent

$\mathrm{L}=0$ Strength function $\quad=2.2 \mathrm{E}-4$, energy independent

$L=1$ Strength function $\quad=0.252 \mathrm{E}-4$, energy independent

$\mathrm{L}=2$ Strength function $\quad=1.45 \mathrm{E}-4$, energy independent

Average capture cross section uncertainty at energy $E$

E (keV) Uncertainty (\%)

$\begin{array}{rr}100 & 11 \\ 90 & 8 \\ 45 & 8 \\ 22.5 & 14 \\ 10 & 11 \\ 4.5 & 17\end{array}$

Resonance integral (capture) 522 b calculation, $490 \pm 50$ b measurement (Ref. 6).

File 3. Neutron Cross Sections

MT=1. Total Cross Section

The total cross section was evaluated using a least squares spline fit to experimental data for this isotope. Spline fits of experimental data for natural tungsten were also factored into the evaluation (see MAT 1129 for Refs). The total cross section curve was smoothly joined to the evaluated total cross section in the unresolved resonance range below $100 \mathrm{keV}$. Isotopic data references are Whalen ${ }^{7}(100-550 \mathrm{keV})$, Martin ${ }^{8}(0.65-20 \mathrm{MeV})$, and Foster and Glasgow ${ }^{9}(2.5-15 \mathrm{MeV})$. General references for the total cross section are Goldberg et $\mathrm{al}^{2}$ and Devaney and Foster. ${ }^{10}$ The uncertainty in the total cross section is probably less than $7 \%$ over the energy range from $600 \mathrm{keV}$ to $20 \mathrm{MeV}$. Between $300 \mathrm{keV}$ and $600 \mathrm{keV}$ the uncertainty increases to $10 \%$ and from 100 to 200 $\mathrm{keV}$ the uncertainty is estimated to be $15 \%$.

\section{MT $=2$. Elastic Cross Section}

The elastic cross section was obtained by subtracting the nonelastic cross sections from the evaluated total cross section. The elastic cross section is in good agreement with the data of Lister ${ }^{11}$ between $300 \mathrm{keV}$ and $0.7 \mathrm{MeV}$. Between $0.7 \mathrm{MeV}$ and $1.5 \mathrm{MeV}$, the elastic cross section is some $10 \%$ higher than the experimental data. Between $1 \mathrm{MeV}$ and $9 \mathrm{MeV}$ the evaluated curve lies above 2PLUS-COMNUC results. At $4.3 \mathrm{MeV}$ the cross section is some $15 \%$ lower than the experimental data point of Kinney and Perey ${ }^{12}$ for natural tungsten. Our evaluation is, however, in agreement with their data above $5 \mathrm{MeV}$. 
$\mathrm{MT}=4$. Total Inelastic Cross Section

Equal to the sum of the level excitation and continuum cross section. The total inelastic cross section for this isotope is in general agreement with the data of owens et al. ${ }^{13}$ for natural tungsten between 5 and $7 \mathrm{MeV}$.

$M T=16 . \quad(n, 2 n)$ Cross Section

The semi-empirical techniques of Pearlstein ${ }^{14}$ and W. D. Lu et a1.15 were used to deduce the $n, 2 n$ cross section. The evaluated curve at $14.8 \mathrm{MeV}$ is in agreement with Druzhinin et al. ${ }^{16}$

$\mathrm{MT}=17 . \quad(\mathrm{n}, 3 \mathrm{n})$ Cross Section

The $n, 3 n$ cross sections were deduced using the semi-empirical techniques of S. Pearlstein ${ }^{14}$ and W. D. Lu. ${ }^{15}$ An effective threshold for the $n, 3 n$ reaction was set $1.25 \mathrm{MeV}$ above the theoret:ical threshold.

$\mathrm{MT}=28 . \quad(\mathrm{n}, \mathrm{pn})$ Cross Section

The shape of the $n, p n$ excitation curve is based upon the experimental data of J. F. Barry et al.17 The uncertainty in the cross section is $25 \%$ at energies well above the observed threshold. The cross section contains contributions from the $n, n p$ and $n, d$ reactions.

$\mathrm{MT}=51$. Inelastic Excitation (Direct and Compound Nucleus) to the $2^{+}$Leve1

The cross sections were calculated using 2PLUS-COMNJC. They have been renormalized via a least squares fit to data of $D$. Lister et al. ${ }^{18}$ with a $10 \%$ uncertainty in the adjusted curve.

MT=52-59, 91. Inelastic Excitation (Compound Nucleus On1y) .

Calculation by the COMNUC code for the $401,730,840,850,960$, 1040,1110 , and $1250 \mathrm{keV}$ levels plus the continuum. The 401, 730, and $942-\mathrm{keV}$ level excitation curves were renormalized to the data of D. Lister. ${ }^{18}$ The uncertainty in the adjusted cross sections is about 10\%. The sum of the 840 and $850 \mathrm{keV}$ level CoMNuC results were renormalized to the $863 \pm 10 \mathrm{keV}$ excitation data of $D$. Lister. Likewise, the sum of the 1040 and $1110 \mathrm{keV}$ levels were renormalized to the $1035 \pm 10 \mathrm{keV}$ experimental data. The summed levels have a $15 \%$ cross section uncertainty.

$\mathrm{MT}=102$. Radiative Capture Cross Section

The radiative capture cross section was evaluated between $10 \mathrm{keV}$ and $4 \mathrm{MeV}$ using a least squares spline fit to experimental data (see $M F=2, M T=151$ ). Comparisons with CoMNuc theoretical results were excellent. Adjustments were made, however, to the data fit 
in the neighborhood of the $2^{+}$threshold to better fit the theoretical calculations. These adjustments improved the reconstructed elemental file comparison with experiment. Above $4 \mathrm{MeV}$ the data fit was joined to theoretical capture cross sections calculated between 4 and $20 \mathrm{MeV}$. The theory of Benzi and Reffolg was used to determine the collective and direct interaction cross section.

MT=103. $\quad(n, p)$ Cross Section

The $n, p$ cross section evaluation is based upon experimental data of J. F. Barry et a1. ${ }^{17}$ Uncertainty is $25 \%$ at energies wel1 above the observed threshold.

$M T=107 . \quad(n, \alpha)$ Cross Section

The $n, \alpha$ cross section evaluation is based upon data of A. Rubino and D. Zubke ${ }^{20}$ for ${ }^{181} \mathrm{Ta}$. The few experimental data points for isotopes of tungsten between 14 and $15 \mathrm{MeV}$ qualitatively substantiate the use of this curve. The evaluation has not been extended below $11 \mathrm{MeV}$.

\section{File 4. Neutron Angular Distributions}

MT=2. Elastic Angular Distributions

The angular distributions for elastic scattering are given as Legendre polynomial coefficients in the $\mathrm{cm}$ system. These data were calculated by 2PLUS-COMNUC codes for each isotope. As littlc isotopic experimental data existed, the calculated data were averaged according to abundance and compared to natural tungsten data. The comparison indicated that the experimental data contained direct inelastic reactions to the first level. With the calculated first-level direct inelastic included, agreement within experimental error was generally indicated over all energies. This agreement also supports the validity of the inelastic angular file below. The natural tungsten experimental data were taken from Refs. 21-28. Error estimates for this section are difficult because it appears that pure elastic scatter has not been measured except at low energies. Because of the good agreement overall with the combined data, this file is estimated to be accurate to $15 \%$ at energies below $1 \mathrm{MeV}$, and within a factor of two at energies above $1 \mathrm{MeV}$ for all scattering angles.

MT=51-59, 91. Angular Distribution of Inelastic Scattered Neutrons

Angular distributions are given as Legendre polynomial coefficients in the laboratory frame of reference. These data are based upon 2PLUS-COMNUC calculations. They include anisotropic contributions from direct inelastic excitation of the $2^{+}$level. Compound level and continuum excitations are isotropic in the laboratory system. Errors are small simply due to the predominance of isotropy. 


\section{File 5. Neutron Energy Distributions}

$M T=16,17$. Nuclear Temperatures for the $(n, 2 n)$ and $(n, 3 n)$ Reactions

Temperature of first emitted neutron same as for $M T=91$. Temperatures of second and thiri emitted neutrons deduced by conservation of energy assuming the kinetic energy of an emitted neutron is $2 * \mathrm{KT}$.

MT=91. Nuclear Temperature for Inelastic Continuum

References 29-32 were used in deriving temperatures. Least squ. es Weisskopf level spacing was fit to natural tungsten data. Isotopic data adjusted using deformed nucleus level density formula of Gilbert and Cameron. ${ }^{32}$

File 12. Gamma Ray Multiplicities

MT=102. Multiplicities for Gamma Ray Production f rom Radiative Capture

Multiplicities at neutron energies below $1 \mathrm{eV}$ are based on a preliminary thermal measurement for natural $\mathrm{W}$ by Jurney ${ }^{33}$ and from $1 \mathrm{eV}$ to $1000 \mathrm{eV}$ on calculations by Yost et al. ${ }^{34}$ at higher energies the multiplicities are based on an analysis of natural $\mathrm{W}$ measurements (Dickens, ${ }^{36} \mathrm{E}_{\mathrm{n}}=1-20 \mathrm{MeV}$ ) using statistical calculations similar to those described by Troubetzkoy, ${ }^{35}$ with the multiplicities chosen to give rough agreement with the measurements. The theory was used at aj.1 energies to divide the natural $W$ data into isotopic components.

File 13. Gamma Ray Production Cross Sections

$M T=4$. Gamma Ray Production Cross Sections from Inelastic, $(n, 2 n)$, and $(n, 3 n)$ Reactions

The cross sections for discrete photons are based on the level excitation cross sections in $M T=3, M T=51-59$, mainly using the level decay scheme of Gove. ${ }^{37}$ The cross sections for continuum gamma rays are based on a statistical theory analysis of the Dickens ${ }^{36}$ measurements on natural $\mathrm{W}$. The theory was used for interpolation, smoothing, and separation of the data into isotopic components.

File 14. Gamma Ray Angular Distributions

$M T=4$, 102. Angular Distributions of Gamma Rays f rom Radiative Capture, Inelastic, $(n, 2 n)$, and $(n, 3 n)$ Reactions

Gamma rays from all reactions are assumed isotropic in the laboratory system. 


\section{File 15. Gamma Ray Energy Distributions}

MT=4. Energy Distributions of Gamma Rays from Inelastic, $(\mathbf{n}, 2 \mathrm{n})$, and $(n, 3 n)$ Reactions

The spectra at all energies are based on a statistical theory analysis of the Dickens ${ }^{36}$ measurements with natural $\mathrm{W}$. The theory was used as described above $(M F=13, M T=4)$. Arbitrary adjustments were made to the theoretical fits in regions where agreement with experiment was poor (mainly below $E_{\mathfrak{n}}=5 \mathrm{MeV}$ ).

\section{MT=102. Energy Distributions of Gamma Rays from Radiative Capture}

Spectra at neutron energies below $1 \mathrm{eV}$ are based on a preliminary thermal measurement by $\mathrm{Jurney}^{33}$ and from $1 \mathrm{eV}$ to $1000 \mathrm{eV}$ on calculations by Yost et al. ${ }^{34}$ At higher energies the spectra are based on statistical calculations using parameters obtained by analyzing the Dickens ${ }^{36}$ measurement for $\Sigma_{\mathrm{n}}=1-3 \mathrm{MeV}$. The theory was used at all energies to divide the natural $\mathrm{W}$ data into separate isotopic components.

(Note: No information is provided in this evaluation on electron production from internal conversion. Such information might be important for local heating and radiation damage problems.)

\section{REFERENCES}

1. A. H. Wapstra and N. B. Gove, Nuc1. Data Tables 9, 267 (1971).

2. M. D. Goldberg et al., BNL-325, 2nd Edition, Supplement 2, (1966).

3. Z. M. Bartolome et al., Nuc1. Sci. Eng. 37, p. 137 (1969).

4. F. J. Rahn and H. Camarda, personal communication, Columbia (1970).

5. J. L. Cook et al., AAEC/TM-392 (1967).

6. M. Drake, (1968) for American Institute of Phys. Handbouk (1972).

7. $\therefore$ F. Whalen, ANL-7210, 16 (1966).

8. R. C. Martin, PhD Thesis, RPT (1967).

9. D. G. Foster and D. W. Glasgow, Phys. Rev. 3C, 576 (1971).

10. J. J. Devaney and D. G. Foster, Jr., Los Alamos report LA-4928 (1972).

11. D. Lister et a1., ANL-7288 (1967).

12. W. E. Kinney and F. G. Perey, ORNL-4803 (1973).

13. R. O. Owens et al., Nucl. Phys. Al12, 337 (1968).

14. S. Pearlstein, Nucl. Sci. Eng. 23, 238 (1965).

15. W. D. Lu et al., Phys. Rev. Cl, 350 (1970).

16. Druzhinin et a1., Sov. J. Nucl. Phys. 4, 515 (1966).

17. J. F. Barry et al., Proc. Phys. Soc. $\overrightarrow{74}, 632$ (1959).

18. D. Lister et a1., Phys. Rev. 162, 1077 (1967).

19. V. Benzi and G. Reffo, CCDN-NW10, ENEA Neutron Compilation Center (1969).

20. A. Rubino and D. Zubke, Nucl. Phys, 85, 606 (1966).

21. A. B. Smith and P. A. Moldauer, BAPS $\underline{5}, 409$ (1960).

22. F. T. Kuchnir et al., Phys. Rev. 176, 1405 (1968).

23. W. Walt and H. Barschal1, Phys. Rev. 93, 1062 (1954).

24. K. Tsukada et al., Nucl. Phys. A125, 641 (1966).

25. R. W. Hill, Phys. Rev. 109, 2105 (1958). 
26. W. Kinney, private communication (1972).

27. D. B. Thomson, Phys. Rev. 129, 1649 (1963).

28. H. Nauta, Nuc1. Phys. 2,124 (1956).

29. S. G. Buccino et al., Nucl. Phys. 60, 17 (1964).

30. R. 0. Owens and J. H. Towle, Nucl. Phys. A112, 337 (1968).

31. W. E. Kinney, ORNL, private communication (1972).

32. A. Gilbert and A. G. W. Cameron, Can. J. Phys. 43, 1446 (1965).

33. E. T. Jurney, private communication (1973).

34. K. J. Yost, J. E. White, C. Y. Fu, and W. E. Ford, Nucl. Sci. Eng. 47, 209 (1972).

35. E. S. Troubetzkoy, Phys. Rev. 122, 212 (1961).

36. J. K. Dickens, private communication (1972).

37. N. B. Gove, Nuclear Data B1-2, 1 (1966). 
SUMMARY DOCUMENTATION FOR ${ }^{233_{U}}$

\author{
by \\ L. Stewart, D. G. Madland, and P. G. Young \\ Los Alamos Scientific Laboratory \\ Los Alamos, New Mexico \\ and \\ L. Weston and G. de Saussure \\ Oak Ridge National Laboratory \\ Oak Ridge, Tennessee \\ and \\ F. Mann \\ Hanford Engineering and Development Laboratory \\ Richland, Washington \\ and \\ N. Steen \\ Bettis Atomic Power Laboracory \\ West Mifflin, Pennsylvania
}

I. SUMMARY

A new evaluation of neutron-induced reactions on ${ }^{23}{ }^{3} \mathrm{U}$ was carried out for Version $V$ of ENDF/B (MAT 1393). The analysis was divided among several laboratories. The thermal data evaluation was performed at BAPL and LASL, the resolved and unresolved resonance regions were evaluated at ORNL and HEDL, and evaluation of the data from $10 \mathrm{keV}$ to $20 \mathrm{MeV}$ and assembly of the composite file was carried out at LASL. In addition, fission product yield data were provided by the CSEWG Yield Subcommittee ( $T$. England, chairman), and radioactive decay data by C. Reich (INEL). Partial documentation of the evaluation is provided in $\mathrm{LA}-7200-\mathrm{PR}^{3}$ and in reference 16 . The evaluation covers the energy range $10^{-5} \mathrm{eV}$ to $20 \mathrm{MeV}$. Gamma-ray production and covariance data will be added to the file in a later MOD 1 update.

II. ENDF/B-V FILES

File 1. General Information

MT $=451$. Descriptive data. 
MT=452. $\bar{v}$ Total

Sum of prompt plus delayed $\bar{v}$. Used thermal value recommended by CSEWG Standards Subcommittee of 2.4947 on 5-27-78. This value is $1 \%$ larger than recommended by Lemme $1^{1}$ and $0.13 \%$ smaller than Version IV.

MT=455. $\quad \vec{v}$ Delayed

The delayed yialds and spectra were evaluated by Kaiser and Carpenter at ANJ-Idaho (see Ref. 2 for technical details). The same six-group yields appear in Version IV but the spectra have been changed for Version $V$.

MT=456. $\bar{v}$ Prompt

The energy dependence of prompt $\bar{v}$ is changed significantly from Version IV. Although the thermal value is slightly lover, the value around $1.5 \mathrm{MeV}$ is significantly higher and has a different shape with three slightly different slopes. This evaluation relies heavily on the measurements with respect to ${ }^{235} \mathrm{U}$ and ${ }^{252} \mathrm{Cf}$ using the CSEWG recommended standards. In particular, the data of Boldeman, Sergachev, Nurpeisov, and Block ${ }^{5-7}$ were weighted at all energies while the Mather ${ }^{8}$ measurements were relied upon only at high energies. Smirenkin, Flerov, and Protopopov ${ }^{9-11}$ also contributed in the high-energy range. This evaluation decouples the thermal value based on $\eta$ measurements from the fast range. Otherwise, the $\bar{v} \eta$ discrepancy would be perpetuated to $20 \mathrm{MeV}$.

MT=458. Energy Release in Fission

These values were: taken directly from an evaluation by $R$. Sher ${ }^{12}$ (Stanford).

\section{File 2. Resonance Parameters}

\section{$\mathrm{MT}=151$. (a) Resolved Resonance Region}

Resolved range extends to $63 \mathrm{ev}$. Multi-level parameters provided by de Saussure (ORNL) in Adler-Adler formalism from analysis by Reynolds (KAPL). Version IV used single-level Breit-Vigner representation but had large fluctuations in fission in File 3 background.

\section{(b) Unresolyed Resonance Region}

Unresolved range extends to $10 \mathrm{keV}$. Version IV was pointwise over this range. A reevaluation of the point-wise cross sections begun by Mann (HEDL) were used as the starting point by Weston (ORNL), who obtained new average cross sections for fission and capture which require no File 3 backgrounds. Cross sectious based on Carlson and Behreens, ${ }^{4}$ Weston, ${ }^{17}$ Cao, ${ }^{18}$ and Nizamuddin ${ }^{19}$ for fis- $^{2}$ sion. Weston's data ${ }^{20}$ were used for capture using a potential 
scattering radius of 0.9893 . Reasonable agreement with Pattenden's total cross section ${ }^{2}$ was obtained, especially below $1 \mathrm{keV}$. New measurements are needed in this range.

File 3. Neutron Cross Sections

Thermal Range

The $2200 \mathrm{~m} / \mathrm{s}\left(E_{\mathrm{n}}=0.0253 \mathrm{eV}\right)$ data are as follows:

\begin{tabular}{|c|c|c|c|}
\hline $\begin{array}{l}\text { Eta } \\
\text { Alpha } \\
\bar{v} \text { Prompt } \\
\bar{\nu} \text { Total }\end{array}$ & $\begin{array}{l}2.2959 \\
0.0866 \\
2.4873 \\
2.4947\end{array}$ & $\begin{array}{l}\text { Capture } \\
\text { Fission } \\
\text { Absorption } \\
\text { Elastic } \\
\text { Total }\end{array}$ & $\begin{array}{c}45.76 \\
528.45 \\
574.21 \\
12.6 \\
586.81\end{array}$ \\
\hline
\end{tabular}

The capture and fission cross sections were renormalized by $N$. Steen (BAPL). The elastic scattering in the thermal range changed significantly from Version IV to conform to Leonard's thermal and Weston's evaluation in unresolved range. The total was adjusied accordingly. Elastic and total changes made by LASL.

$10 \mathrm{keV}-20 \mathrm{MeV}$

Much of the File 3 data above $50 \mathrm{keV}$ relied heavily on model calculations performed by Madland (LASL). Calculations were particularly important for this isotope since experimental information was often insufficient if not completely missing. A detailed description of the methods used can be found in reference 3. Note that for covenience, many of the specific references found in BNL-325 are not repeated here.

MT $=1$. Total Cross Section

No measurements exist from 10 to $40 \mathrm{keV}$; therefore, an extrapolation was made to give reasonable agreement with recent ANL data ${ }^{3}$ above $40 \mathrm{keV}$ and cross sections predicted by Madland. The present evaluation is based on recent ANL measurements ${ }^{13}$ and earlier work of Green (Bettis) and Foster (Hanford). See BNL-325 (Ref. 5). No measurements exist above $15 \mathrm{MeV}$. This reevaluation resulted in an increased total cross section up to $7 \mathrm{MeV}$, the increase near $1.6 \mathrm{MeV}$ being as large as $8 \%$. Use of the Foster data resulted in a decrease of $4.8 \%$ near $14 \mathrm{MeV}$.

MT=2. Elastic Cross Section

(Dbtained from total minus reaction). The increased total cross section required a significant increase in the elastic cross section over the energy range to $7 \mathrm{MeV}$. At $14 \mathrm{MeV}$ the elastic was decreased due to the decrease in the total. 
$\mathrm{Mr}=4$. Inelastic Cross Section

Sum of MT=51-54 and MT=91. These data were taken from modeI calculations of discrete and continuum compound inelastic scattering and coupled-channel direct inelastic scattering.

$M T=16,17 .(n, 2 n)$ and $(n, 3 n)$ Cross Sections

Taken from Hauser-Feshbach statistical model calculations performed by Madiland.

$\operatorname{MT}=18$. ( $(n, f)$ Cross Sections

Data from $10 \mathrm{keV}$ to $100 \mathrm{keV}$ sparse and reasonably discrepant. Above $100 \mathrm{keV}$, we relied heavily on the ratio measurements of Carlson and Behrens ${ }^{4}$ and those of Meadows, ${ }^{14}$ normalized to Version $V^{235} U$ fission. The absolute data of Poenitz ${ }^{15}$ were also employed, although good agreement among the sets was lacking. The evaluated curve was drawn as smoothly as possible duc to the magnitude of the discrepancy among the sets.

MT=51-54. Discrete Inelastic Cross Sections

Taken from compound and direct inelastic scattering model.

MT=102. Radiative Capture Cross Section

No data exist above $10 \mathrm{keV}$ except for the $\alpha$ measurements of

Diven and Hopkins (see Ref. 5) which extend to $1 \mathrm{MeV}$.

Extrapolated to $20 \mathrm{MeV}$ assuming a rise due to direct capture.

$\mathrm{MT}=251,252,253 . \mu_{\mathrm{L}}, \xi, \gamma$

Calculated from $M F=3$ and 4 data and input by Kinsey at BNL.

File 4. Neutron Angular Distributions

All neutron angular distributions isotropic in laboratory system except for the elastic (MT=2) which was taken from Version IV. The elastic and direct inelastic should be modified in the next update.

File 5. Neutron Energy Distributions

$M T=16,17 .(n, 2 n),(n, 3 n)$ Energy Distributions

Represented by an evaporation spectrum with $\mathrm{LF}=9$.

MT=18. ( $n, f)$ Neutron Energ $y$ Distributions

Represented by an energy-dependent Watt spectrum with an average energy at thermal of $2.073 \mathrm{MeV}$. This spectrum based on Grundl ratio data to ${ }^{235} \mathrm{U}$ and ${ }^{239} \mathrm{Pu}$. 
$M T=91$. Inelastic Continuum Energy Distributions

Represented by an evaporation spectrum with $L F=9$.

$\mathrm{MT}=$ 455. Delayed Neutron Spectra

Evaluated in six time groups by Kaiser and Carpenter ${ }^{2}$ at ANLIdaho.

File 8. Fission Product Yields and Decay Data

MT=454. Individual Fission Product Yields

Direct yields before neutron emissior.

MT=459. Cumulative Yields

Cumulative yields along each isobaric chain after neutron emission taken from set 5D.3/78. Values recommended by CSEWG Yields Subcominit tee (England, chairman).

Note: Both direct and cumulative yields are normalized by the same factors based on B. F. Rider evaluation. The isomeric state model, LA-6595-MS (ENDF-241), and delayed neutron emission branchings ( $P n$ values) for 102 emitters, and pairing effects, LA-6430MS (ENDF-240), have been incorporated.

Uncertainties are based on the total yield to each ZA. When there is an isomeric state, the independent nuclide yield to each state has a larger uncertainty than the total yield in state distributions. (Uncertainties average $50 \%$ but can be larger). Any yield with an uncertainty of $45-64 \%$ may be model estimate or a value assigned in the wings of the mass distribution. These small yields may be accurate only within a factor of two.

Data prepared for ENDF/B-V by T. R. England (Ref. LA-UR-78-687). MT=457. Radioactive Decay Data

Evaluated by C. Reich (INEL).

Q (ALPHA) - 1974 Version of Napstra-Bos-Gove mass tables.

Half-life - Average of values by Vaninbroukx ${ }^{22}$ and Jaffy et a1. ${ }^{3}$

Alpha Energies and Intensities - Are based mainly on the results of Ellis $^{24}$ with a few deletions based on level-scheme considerations. Energies and intensities of the two most prominent alpha groups are those recommended by Rytz. ${ }^{25}$

Gamma-Ray Energles and Intensities - Based on results of Rroger and Reich. 25 


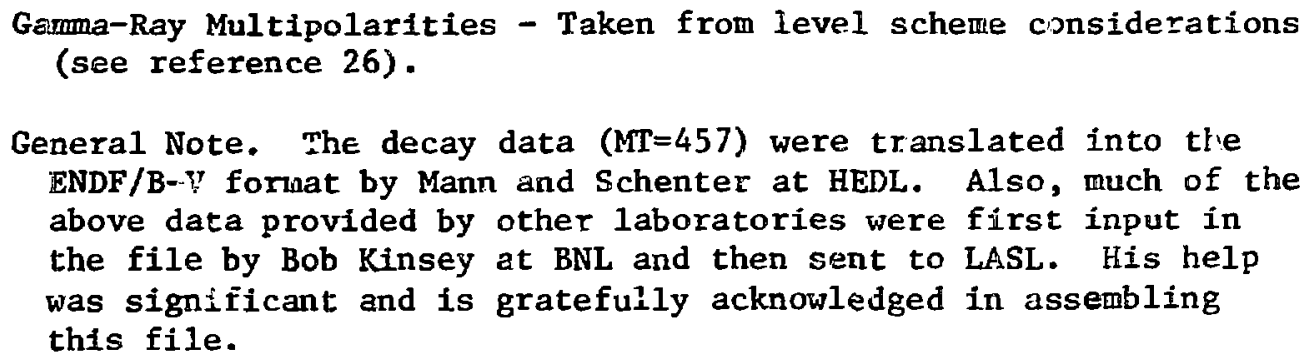

\section{REFERENCES}

1. H. D. Lemmel, Proc. of a symposium on Neutron Standards and Applications held at NBS on March 28-31, 1977. See p. 170.

2. Kaiser and Carpenter, private communication to BNL (1978).

3. D. G. Madland and P. G. Young, LA-7200-PR (1978) p. 11 and p. 13.

4. G. W. Carlson and J. W. Behrens, Nucl. Sci. Eng. 66, 205 (1978).

5. D. I. Garber and R. R. Kinsey, BNL-325, Vol. 11 (1976).

6. J. W. Boldeman, J. Hucl. Ener. 25, 321 (1971), and Boldeman, Bertram, and Walsh, Nucl. Phys. A265, 337 (1976).

7. L. Reed, R.W. Hockenbury, and R. C. Block, report Co0-3058-39, p. 9 (Sept. 1973).

8. D. S. Mather, Nuc. Phys. 66, 149 (1965).

9. G. N. Smirenkin, At. Ezer. 4, 88 (1958).

10. N. N. Flerov, At. Ener. 10, 86 (1961).

11. A. N. Protopopov, At. Ener. 5, 71 (1958).

12. R. Sher, S. Fiarman, and C. Beck, private communication to BNL (1976).

13. W. P. Poenitz, J. F. Whalen, P. Guenther, and A. B. Smith, Nucl. Sci. Eng. $\underline{68}, 358$ (1978).

14. J. W. Meadows, Nucl. Sci. Eng. 54, 317 (1974).

15. W. P. Poenitz, ANL-NDM-36 (1978).

16. L. Stewart, D. Madland, and P. Young, Trans. Am. Nuc. Soc. 28, 721 (1978).

17. L. Weston et al, Nucl. Sci. Eng. 42, 143 (1970).

18. M. Cao et al., J. Nucl. Ener. 24, 111 (1970).

19. S. Nizamuddin and J. Blons, Nucl. Sci, Eng. 54, 116 (1974). 
20. L. Weston et al., Nucl. Sci. Eng. 34, 1 (1968).

21. N. Pattenden et al, Nucl. Sci. Eng. 17, 404 (1963).

22. R. Vaninbroukx et al., Phys. Rev. C13, 315 (1976).

23. A. H. Jaffy et al., Phys. Rev. C9, 1991 (1974).

24. Y. A. Ellis, Nucl. Data Sheers B6, \#3, 257 (1971).

25. A. Rytz, At. Data and Nucl. Data Tables 12, \#5, 479 (1973).

26. L. A. Kroger and C. W. Reich, Nucl. Phys. A259, 29 (1976). 
SUMMARY DOCUMENTATION FOR ${ }^{242} \mathrm{Pu}$

by

F. Mann and $R$. Schen $\iota s$

Hanford Engineering and Developnent Laboratory

Richland, Washington

and

D. G. Madland and P. G. Young

Los Alamos Scientific Laboratory

Los Alamos, New Mexico

\section{SUMMARY}

A new evaluation of neutron-induced reactions on ${ }^{242} \mathrm{Pu}$ was performed for Version $V$ of ENDF/B (MAT 1342). The analysis was divided between HEDL, where the resolved and unresoived resonance regions were evaluated, and LASL, where the data above a neutron energy of $10 \mathrm{keV}$ were evaluated. These evaluations are documented in HEDL-TME-77-54 (Ma77B) and LASL-7533-MS (Ma78). Additionally, decay data were provided by C. Reich (INEL), thermal data by R. Berijamin (SRL), and gamma-ray production data by $R$. Howerton (LLL). The evaluition covers the energy range $10^{-5} \mathrm{eV}$ to $20 \mathrm{MeV}$ and includes covariance data $a i$ all energies.

\section{ENDF/B-V FIIES}

File 1. General Information

MiT=451. Descriptive data

MT=452. $\bar{v}$ Total

Sum of MT $=456$ and a delayed $\bar{v}$ of 0.015 from the measurement of Kr70 as compiled by Ma72.

MT=456. $\bar{v}$ Prompt

Based on a fit to ${ }^{240} \mathrm{Pu}$ experimental data by Fr74 using sys cematics to infer delta $\bar{v}$ to ${ }^{242} \mathrm{Pu}$, and renormalized to ${ }^{252} \mathrm{Cf}$ thermal $\bar{v}$ of 3.757 .

MT=458. Energy of Fission

Based on work of $\operatorname{Sh} 76$. 


\section{File 2. Resonance Parameters $\left(E_{\mathrm{n}}=0-10 \mathrm{keV}\right)$}

\section{$\mathrm{MT}=151$. (a) Resolved Resonances}

The resolved resonance region covers the energy range $0-986 \in \mathrm{V}$. One bound level and 67 resolved resonances describe the cross section data from zero to $986 \mathrm{eV}$. Except for the bound and $2.68 \mathrm{eV}$ levels, parameters are from BNL-325 (Mu76). Parameters for the bound and $2.68 \mathrm{eV}$ levels have been modified to preserve the cross section values and shapes in the thermal region as described by Yo70 and Yo71, along with the higher resonance capture integral suggested by integral and production experiments (Bu57, Ha64, and Be75).

\section{(b) Unresolved Resonances}

The unresolved resonance region covers the energy range from $986 \mathrm{eV}$ to $10 \mathrm{keV}$. Average parameters obtained by averaging the resolved resonance data were used for the $\mathrm{L}=0$ resonances. The remaining data were obtained from MAT 1161, ENDF/B-IV.

\section{File 3. Neutron Cross Sections}

\section{Genera1}

Evaluation from 0.01 - $20 \mathrm{MeV}$ described in Ma78. Statistical compound nucleus and direct reaction theory calculations performed with LASL versions of COMNUC (Du70, 3-29-78 version) and JURARL (Re71). All calculations used LASL preliminary global actinide optical potential (Ma77). Complete set of calculations performed but elastic and fission cross section evaluations differ slightly (less than 5\%) from calculations because of influence of fission measurements. $(n, f),(n, n f)$, and $(n, 2 n f)$ cross sections calculated subject to constraint that their sum equals measured (Be78) total fission cross section within 5\%. Discrete fission channels (up to 12) and deformed level density continuum fission channels used.

$\mathrm{MT}=1$. Total Cross Section

Spherical optical model calculation with nuclear deformation effects accounted for by coupled-channel calculations of up to 5 states of ground state band.

\section{MT $=2$. Elastic Cross Section}

Difference between $M T=1$ and $M T=4,16,17,18$, and 102, Agrees with model calculation to within few per cent.

$M T=4$. Inelastic Scattering Cross Section

Sum of $M T=51-69$ and $M T=91$. 
$M T=16,17(n, 2 n)$ and $(n, 3 n)$ Cross Sections

Based on compound nucleus statistical model calculations.

$\mathrm{MT}=18$. Fission Cross Sections

Beiow $100 \mathrm{keV}$ based on experimental data of Au7l. From 0.1 to 20 $\mathrm{MeV}$ based on experimental data of Be78 (see Figs. 1 and 2).

MT $=51-54 . \quad$ Discrete Inelastic Cross Sections

Based on Hauser-Feshbach compound nucleus calculation and coupledchannel calculation of direct inelastic scattering for first 5 levels of ground state rotational band using deformation parameters of $\mathrm{Be} 73$.

MT=55-69. $\cdot$ Discrete Inelastic Cross Sections

Based on Hauser-Feshbach compound nucleus calculation.

MT $=102$. Capture Cross Sections

Based on compound nucleus statistical calculation with gamma strength function adjusted to agree with Ho75 measurements $\left.2 \pi \Gamma_{\gamma} / \mathrm{D}=0.01045, \mathrm{Mu} 73\right)$. Above $4 \mathrm{MeV}$, semi-direct contribution added from preequilibrium cascade calculation with gamma-ray emission probability, calculated at each stage (Ar78). See Fig. 3 for comparison with measurements.

File 4. Neutron Angular Distributions

MT=2. Elastic Scattering Angular Distributions

Shape elastic component based on deformed optical model calculation. Compound nucleus component assumed isotropic. All distributions given in form of Legendre coefficients.

$\mathrm{MT}=16,17,18 .(n, 2 n),(n, 3 n)$, and $(n, f)$ Angular Distributions

Given as Legendre coefficients and assumed isotropic in the laboratory system.

$\mathrm{MT}=51-54$. Discrete Inelastic Angular Distributions (A)

Direct component taken from deformed optical model calculation, and compound nucleus component assumed isotropic. Given in form of Legendre coefficients.

$M T=55-69$. Discrete Inelastic Angular Distributions (B).

Legendre coefficient representation assumed isotropic in the centerof-mass system. 
$\mathrm{MT}=91$. Continuum Inelastic Angular Distributions

Legendre coefficient representation assumed isotropic in the laboratory system.

File 5. Neutron Energy Distributions

$M T=16,17 . \quad(n, 2 n),(n, 3 n)$ Energy Distributions

Nuclear temperatures calculated from level density parameters used in model calculations (Gi65 and Co67).

MT=18. ( $(n, f)$ Neutron Energy Distributions

Fission Maxwellian using energy-dependent temperatures from Terre11 (Te65).

MT $=91$. Continuum Inelastic Neutron Energy Distributions

Nuclear temperatures calculated from level density parameters used in nuclear model calculations (Gi65 and Co67).

File 8. Fission-Product Yield and Nuclide Decay Data

MT=454. Fission Yield Data

Fission-product yields were obtained from the recommendations of the CSEWG Yields Subcommittee (T. R. England chairman).

$\mathrm{MT}=457$. Decay Data

The Q (alpha) was obtained from the 1974 version of the WapstraBos-Gove mass tables. Half-life values were taken from reference Va74, and other data were obtained from the Table of Isotopes (Le77) and Nuclear Data Sheets (El70). Note that the energies and intensities of the two highest energy alpha groups are those recommended by Rytz (Ry73).

Al1 the decay data were translated into ENDF/B-V format by Mann \& Schenter (HEDT, 6/76).

Files 12-15. Photon-Production Data

Files taken from the LLL evaluations of R. Howerton (Ho76). Files extended to the energy range $1.0^{-5} \mathrm{eV}$ to $20 \mathrm{MeV}$ and merged to this evaluation at BNL by R. Kinsey.

Eile 33. Neutron Cross Section Covariances (HEDL and LASL)

Approximate error files determined from estimated uncertainties in nodel calculations (MT=1, 2, 4, 16, 17, 102) and in experimental measurements (MT $=18,102)$. 


\section{REFERENCES}

Ar78 E. D. Arthur, private communication (1978).

Au71 G. F. Auchampaugh, J. A. Farrel1, and D. W. Bergen, Nucl. Phys. A171, 31 (1971).

Be73 C. E. Bemis et a1., Phys. Rev. C8, 1466 (1973).

Be75 R. Benjamin et al., DP-1394 (1975).

Be78 J. W. Behrens et al., Nuc. Sci. Eng. 66, 433 (1978).

Bu57 J. Butler et al., Can. J. Phys. 35, 147 (1957).

Co67 J. I.. Cook et al., Aust. J. Phys. 20, 477 (1967).

Du70 C. L. Dunford, AI-AEC-12931 (1970).

El70 Y. A. Ellis, Nucl. Data Sheets B4, 非, 683 (1970).

Fr74 J. Frehaut et al., CEA-R-4626 (1974).

Gi65 A. Gilbert and A. G. W. Cameron, Can. J. Phys. 431446 (1965).

Ha64 J. Halperin et al., ORNL-3679, 13 (1964).

Ho75 R. W. Hockenbury et al., NBS Special Publication 425, Vc1. 2, p. 584, (1975).

Ho76 R. Howerton et al., UCRL-50400, Vo1. 15, Part A (Methods, 1975) and Part B (Curves, 1976).

Kr70 M. S. Krick and A. E. Evans, Trans. Am. Nuc1. Soc. 13, 746 (1970).

Le77 C. M. Lederer, Table of Isotopes, $7^{\text {th }}$ Ed (preliminary data received in private communication, 1977).

Ma72 F. Manero and V. Konshin, At. En. Rev. 10, 637 (1972).

Ma77 D. Madland, LA-7066-PR, p. 12 (1977).

Ma78 D. G. Madland and P. G. Young, LA-7533-MS (1978).

Mu73 S. Mughabghab and D. Garber, BNL-325, $7^{\text {th }}$ Ed., Vol. 1 (1973).

Re71 H. Rebel and G. W. Schweimer, KFK-133 (1971).

Ry73 A. Rytz, At. Data and Nucl. Data Tables 12, \#5, 479 (1973).

Sh76 R. Sher, S. Fiarman, and C. Beck, private communication (Oct. 1976).

Va74 R. Vaninbroukx, Euratom report EUR-5194E (1974).

Wi78 K. Wisshak and F. Käppeler, Nucl. Sci. Eng. 66, 363 (1978).

Yo70 T. Young and S. Reeder, Nucl. Sci. Eng. 40, 389 (1970).

Yo71 T. Young et al., Nucl. Sci. Eng. 43, 341 (1971). 


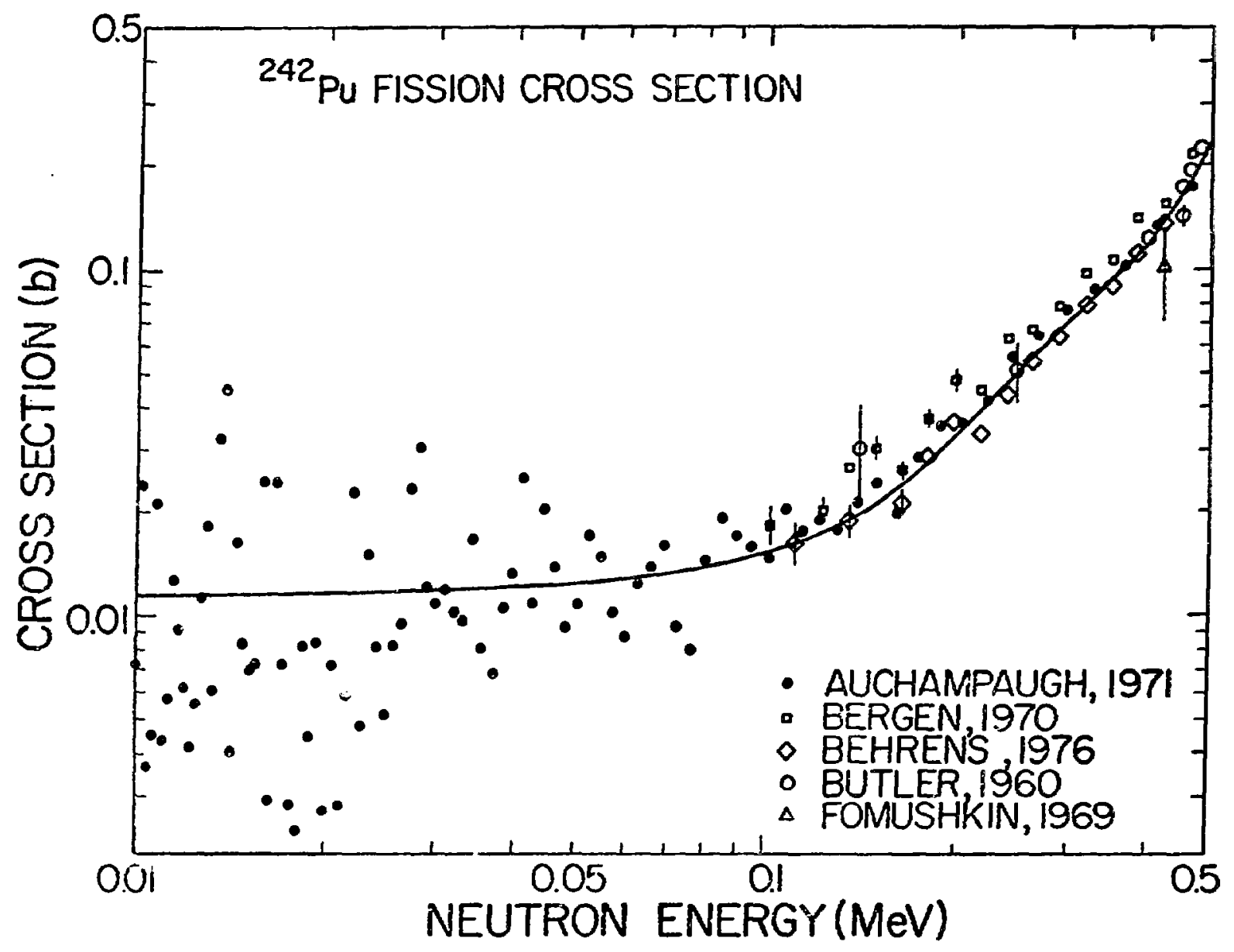

Fig. 1.

Experimental and evaluated cross sections for the ${ }^{242} \mathrm{Pu}(\mathrm{n}, \mathrm{f})$ reaction Exom $10 \mathrm{keV}$ to $0.5 \mathrm{MeV}$. The evaluated curve is based upon the Auchampaugh (Au71) and Behrens (Be76) ratio measurements relative to the ${ }^{235} \mathrm{U}(\mathrm{n}, \mathrm{f})$ reaction. The values shown nere were converted using a preliminary evaluation of the ${ }^{235} \mathrm{U}(\mathrm{n}, \mathrm{f})$ cross section and will be adjusted by up to $4 \%$ in the final evaluation. 


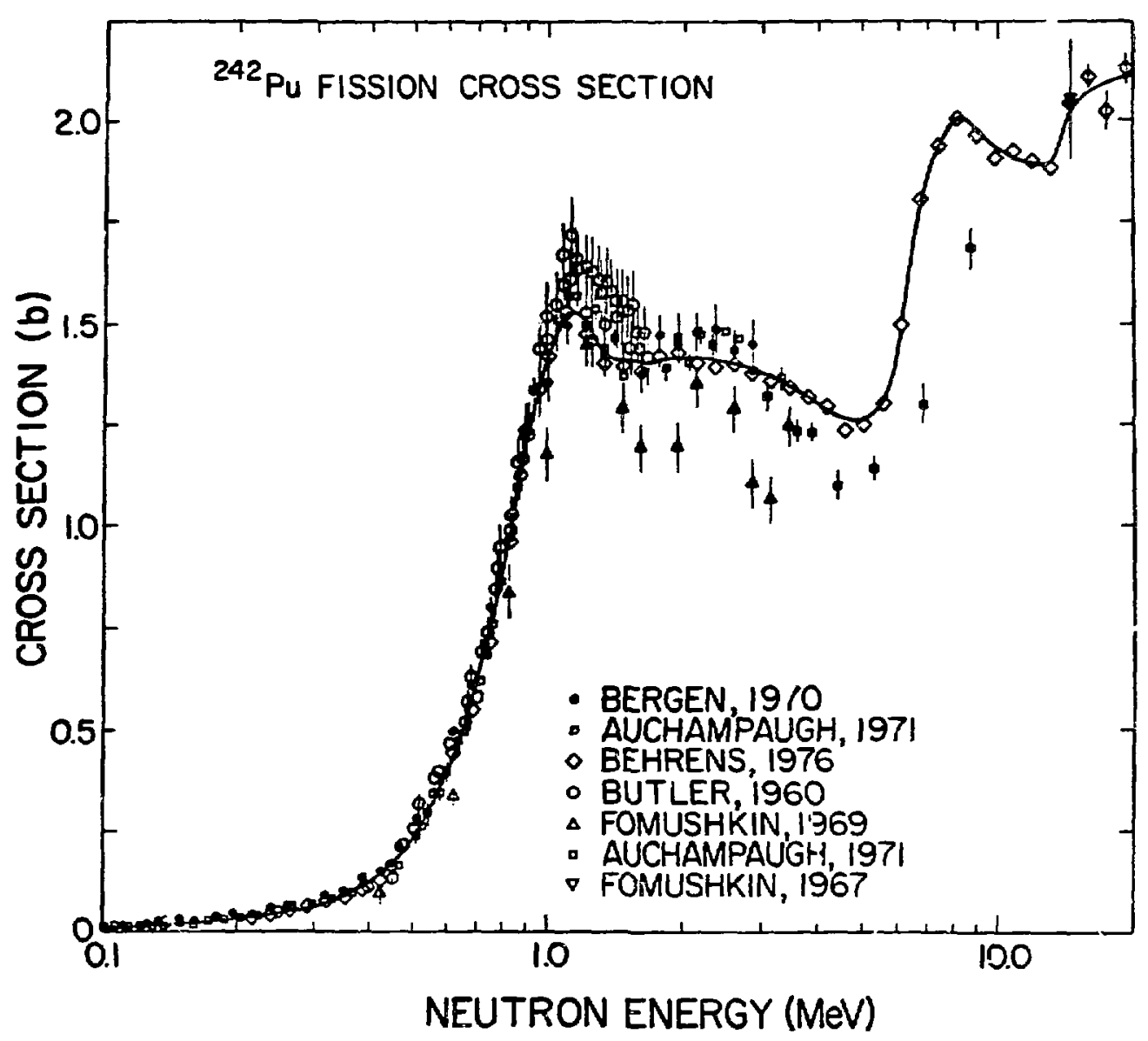

Fig. 2 .

Experimental and evaluated cross sections for the ${ }^{242} \mathrm{Pu}(\mathrm{n}, \mathrm{f})$ reaction from 0.1 to $20 \mathrm{MeV}$. See caption for Fig. 1 . 


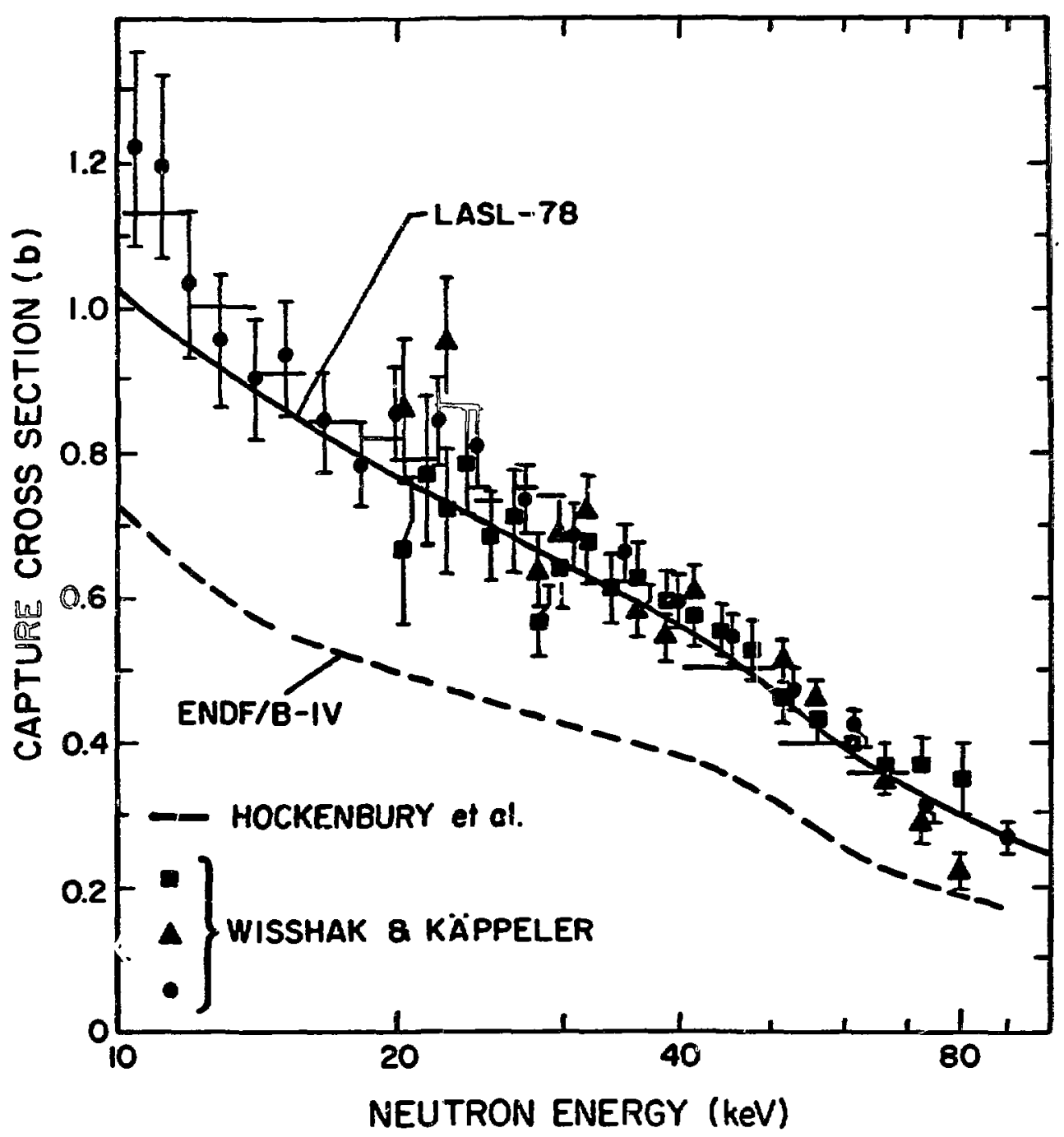

Fig. 3.

Experimental and evaluated cross section for the ${ }^{242} \mathrm{Pu}(\mathrm{n}, \gamma)$ reaction between 10 and $100 \mathrm{keV}$. The experimental data are from Hockenbury (Ho75) and Wisshak and Käpeler (Wi78). 


\begin{abstract}
APPENDIX B
Summary Documentation of LASL Covariance Data Evaluations for ENDF/B-V
\end{abstract}


CROSS SECTION COVARIANCES FOR $1_{\text {hi }}$

by

D. G. Fester, Jr. and P. G. Young

I. Data Summary

Covariance data are included in $M F=33$ for $M T=1,2$, and 102 of the $1_{\mathrm{H}}$ evaluation for the full energy range of $10^{-5} \mathrm{eV}$ to $20 \mathrm{MeV}$. The covariances are developed mainly from two basic components: (1) a short range or localiy correlated component resulting from statistical or localized errors in measurements, and (2) longer range correlated components resulting from systematic errors in the measurements used, or from the analysis procedure itself.

Covariance data are not provided for gamma-ray production data ( $\mathbb{F}=12$, 14) or for neutron angular distributions $(M F=4)$.

\section{Methodology}

a. $\quad \mathrm{MT}=2$

The MT $=2$ cross section is based upon a phase shift analysis of a large body of experimental data. The evaluated covariances are based upon quoted errors in the experimental data base, assuming a relatively high degree of correlation with energy due to the theoretical analysis. The standard deviations in the file range from 0.5 to $1 \%$.

b. $\mathrm{MT}=102$

The MT $=102$ cross section is based upon a very accurate thermal measurement, a $14-\mathrm{MeV}$ measurement, numerous deuteron photodisintegrati. $\mathrm{n}$ measurements, and a theoretical treatment of deuteron photodisintegration. Error estimates were based upon quoted standard deviations from the experimental measurements, which ranged from $\pm 0.6 \%$ at thermal to $\pm 9 \%$ near $20 \mathrm{MeV}$. Strong correlations with energy were assumed because of the use of a theoretical model to represent the experiments over most of the energy range. The covariances included in the file should be regarded as conservative since the use of deuteron photodisintegration theory probably reduces the uncertainty from the experimental values. 
c. $\quad$ MT $=1$

The $M T=1$ covariances are given implicitly (NC-type subsection) over the entire energy range as derived from the sum of $\mathrm{MT}=2$ and $\mathrm{MT}=102$.

d. Correlations Across Reaction Types

Derivation of MT $=1$ from $M T=2$ and 102 leads to correlations of the type $M T=1 ; M T 1=2$, 102 over the entire energy range. These correlations are given implicitly in an NC-type subsection. 
CROSS SECTION COVARIANCES FOR ${ }^{6} \mathrm{Li}_{\mathrm{i}}$

\author{
by
}

G. M. Hale

\title{
I. Data Summary
}

Cross-section covariances intended for use at energies below $1 \mathrm{MeV}$ have been supplied with the Version $V$ ENDF/B evaluation for the reactions important at those energies. Specifically, File 33 data are given for the total, elastic and $(n, t)$ cross sections ( $M F=3 ; M T=1,2$, and 1.05) for incident neutron energies between $10^{-5} \mathrm{eV}$ and $20 \mathrm{MeV}$, although the values in the 1-20 $\mathrm{MeV}$ range are duplicate:s of those around $I \mathrm{MeV}$, and are not intended for use. Covariances are not given at any energies for the neutron angular or energy distributions $(M F=4,5)$, or for the gamma-ray production files $(M F=12,14)$.

\section{Methodology}

The cross-section covariances are derived from covariances of the R-matrix parameters from which the evaluated cross sections are calculated, using firstorder error propogation. The ccvariance between cross section $X$ at energy $E$ and cross section $Y$ at energy $E^{\prime}$ is thus

$$
\operatorname{Cov}\left[X(E), Y\left(E^{\prime}\right)\right]=\left.\sum_{i j} \frac{\partial X(E)}{\partial p_{i}} c_{i j} \frac{\partial Y\left(E^{\prime}\right)}{\partial p_{j}}\right|_{p=\bar{p}},
$$

where $\left\{p_{i}\right\}$ are the R-matrix parameters and $C$ is the matrix of their covariances,

$$
c_{i j}=\left\langle\left(p_{i}-\bar{p}_{i}\right)\left(p_{j}-\bar{p}_{j}\right)\right\rangle
$$

which is known from the curvature of the $\chi^{2}$ surface in the vicinity of the local minimum occurring at $\mathrm{p}=\overline{\mathrm{p}}$. 
The relative covariances are entered directly (using the $L B=5$ represencatica) in bins centered on a 15-point grid of energies between 0.1 and 1000 keV. For compactness, the relative covariances of the total cross section $(M T=1)$ are entered as NC-type sub-subsections, implying that they are to be constructed from those for the $(n, n)(M T=2)$ and $(n, t)(M T=105)$ reactions. This particular choice of "derived" cross section was necessary in order to avoid severe loss-of-significance problems in the derived covariances at low energies where $\sigma_{\mathrm{T}} \approx \sigma_{\mathrm{n}, \mathrm{t}}$ 
CROSS SECTION COVARIANCES FOR ${ }^{10} 0_{B}$

by

G. M. Hale

\section{Data Summary}

Relative cross-section covariances are given for the total, elastir, $\left(n, \alpha_{0}\right)$ and $\left(n, \alpha_{1}\right)$ reactions $(M T=3 ; M T=1,2,780$, and 781$)$, as well as for the total $(n, \alpha)$ reaction $(M T=107)$, for incident neutron energies between $10^{-5}$ and $1 \mathrm{MeV}$. Above $1 \mathrm{MeV}$, the covariances are set equal to zero, and are not intended for use. Covariances are not given at any energies for the neutron angular or energy distributions ( $M F=4,5$ ), or for the gamma-ray production files $(M F=12,14)$.

II. Methodology

Cross-section covariances are derived from covariances of the R-natrix parameters from which the evaluated cross sections are calculated, using firstorder propogation. The covariance between cross section $X$ at energy $E$ and cross section $\mathrm{Y}$ at energy $\mathrm{E}^{\prime}$ is thus

$$
\operatorname{Cov}\left[X(E), Y\left(E^{\prime}\right)\right]=\left.\sum_{i j} \frac{\partial X(E)}{\partial p_{i}} c_{i j} \frac{\partial Y\left(E^{\prime}\right)}{\partial p_{j}}\right|_{p=\bar{p}}
$$

where $\left\{p_{i}\right.$ are the $R$-matrix parameters and $C$ is the matrix of their covarlances,

$$
c_{i j}=\left\langle\left(p_{i}-\bar{p}\right)\left(p_{j}-\bar{p}\right)\right\rangle
$$

which is known from the curvature of the $x^{2}$ surface in the vicinity of tise local minimum occurring at $\mathrm{p}=\overline{\mathrm{p}}$. 
The relative covariances are entered directly (using the LB $=5$ representation) in bins centered on a 12-point grid of energies between 0.20 and 0.95 MeV. For compactness, the relative covariances of the tota? cross section $(M T=1)$ and of the total $(n, \alpha)$ cross section $(M T=107)$ are entered as NC-type sub-subsections. The covariances for MT $=1$ are thus constructed from those for $M T=2,780$, and 781 , and those for $M T=107$ are constructed from those for MT $=780$ and 781. Choosing the total $(\mathrm{MT}=1)$ as a "derived" cross section was necessary in order to avoid severe loss-of-significance problems in the derived covariances at low energies where $\sigma_{\mathrm{T}} \approx \sigma_{\mathrm{n}, \alpha_{0}}+\sigma_{\mathrm{i}, \alpha_{1}}$. 


\section{CROSS SECTION COVARIANCES FOR ${ }^{14} \mathrm{~N}$}

by

P. G. Young and D. G. Foster, Jr.

\section{Data Summary}

Covariance data are included in $\mathrm{MF}=33$ for $\mathrm{MT}=1,2,4,102,103$, and 107 of the ${ }^{14} \mathrm{~N}$ evaluation for the full energy range of $10^{-5} \mathrm{eV}$ to $20 \mathrm{MeV}$. In all instances, the covariances are developed from twc basic components: (1) a short range or locally correlated component resulting from statistical or localized errors in measurements or knowledge of structure, and (2) longer range correlated components resulting from systematic errors in the measurements used, or from the analysis procedure itself.

Covariance data are not provided for $\mathrm{MT}=51-82,104,105,700-704,720-723$, 740-741, and 780-790, although discrete cross sections are given for these reactions in $M F=3$. Similarly, there are no covariances for gamma-ray production data $(M F=12-15)$ or for angular and energy distributions $(M F=4,5)$.

II. Methodology

a. $\mathrm{MT}=1$

The total cross-section analysis was divided into three energy regions: below $10 \mathrm{keV}, 10-500 \mathrm{keV}$, and 0.5-20 $\mathrm{MeV}$. Both the low anu igh energy regions are based upon relatively few measurements. The data in the intermediate region is a composite of many measurements, and the normalization is influenced by the analysis in both the low and high energy regions. The covariances for the total cross section were estimated from the normalizations and shape adjustments that were required to bring the various measurements into agreement. The correlations with energy were estinated from the systematic errors in the measurements used for the three energy ranges. Weak linking correlations were assumed for the analyses in all three energy regions.

b. $M T=102,103$, and 107

Variances were obtained by roughly constructing $\pm 2 \sigma$ curves bounding the experimental data. Correlations with energy were based upon estimates 
of systematic errors in the measarements used for the evaluation. Near thresholds, progressively larger (fractional) uncertainties vere assumed. Estimates of the covariances are probably poorest near the thresholds and where the cross sections are smallest, that is, where the variances are largest.

$$
\text { c. } \mathrm{MT}=2,4
$$

Covariances for MT $=4$ at energies below $10 \mathrm{MeV}$ and for $\mathrm{MT}=2$

above $10 \mathrm{MeV}$ were estimated as described above in Section II.b. In the case of $M T=4$, uncertainties in the individual discrete inelastic reactions were combined to obtain the covariances.

The MT $=2$ cross section was derived below $10 \mathrm{MeV}$ by subtraction of the nonelastic from the total, and $M T=4$ was similarly constructed above $10 \mathrm{MeV}$ from the total, elastic, and reaction cross sections. This information was used to derive the $M T=2$ covariances below $10 \mathrm{MeV}$ and the MT $=4 \mathrm{co-}$ variances above $10 \mathrm{MeV}$, and these data are given explicitly in $\mathrm{MF}=33$. That is, the option to use NC-type subsections to represent derived quantities was not used.

d. Correlations Across Reaction Types

Below $E_{n}=1 \mathrm{keV}$, the $\mathrm{MT}=1$ and 2 cross sections were obtained by fitting the $\mathrm{MT}=1$ measurements with a constant plus $1 / \mathrm{v}$ expression, to represent elastic scattering and capture reactions $(M T=102,103)$, respectively. The resulting correlations between MT $=1$ and MT1 $=2,102,103$ are given explicitly in the file. Similarly, the derivation of $M T=2$ from the other reactions from $1 \mathrm{keV}$ to $10 \mathrm{MeV}$ leads to correlations between $\mathrm{MT}=2$ and $\mathrm{MT} 1=1,4$, $102,103,107$, and the derivation of $M T=4$ above $10 \mathrm{MeV}$ leads to correlations of the type $\mathrm{MT}=4, \mathrm{MT} 1=1,2,10 \mathrm{z}, 103,107$ from 10 to $20 \mathrm{MeV}$. These correlations are also given explicitly in the file, that is, NC-type subsections are not used to represent the derived quantities. Explicit representation of the derived covariances required extensive use of LB $=3$ in the NI-type subsections. 


\title{
CROSS SECTION COVARTANCES FOR ${ }^{16}{ }_{0}$
}

\author{
by
}

P. G. Young and D. G. Foster, Jr.

I. Data Summary

Covariance data are included in $M F=33$ for $M T=1,2,4,103$, and 107

of the ${ }^{16} 0$ evaluation for the full energy range of $10^{-5} \mathrm{eV}$ to $20 \mathrm{MeV}$. In

a11 instances, the covariances are developed from two basic components:

(1) a short range or locally correlated component resulting from statistical or localized errors in measurements or knowledge of structure, and (2) longer range correlated components resulting from systematic errors in the measurements used, or from the analysis procedure itself.

Covariance data are not provided for MT $=51-89,102,104$, and 780-783, although discrete cross sections are given for these reactions in MF $=3$. Similarly, there are no covariances for gamma-ray production data (MF $=12-14$ ) or for angular and energy distributions $(M F=4,5)$.

II. Methodology

a. $E_{n}<6 \mathrm{MeV}$

In this energy range the evaluation of MT $=1,2,107$ was based upon a coupledchannel R-matrix analysis of a composite of experimental data. Covariances were estimated from the normalizations and shape adjustments required to bring the various measurements into agreement, quoted errors for the experimental data, and estimates of the correlations introduced by the R-matrix analysis itself.

b. $E_{n}=6-20 \mathrm{MeV}$

1. $\mathrm{MT}=1$

The evaluated cross section in this region is based upon a composite of several measurements. Covariances were estimated from the normalizations and shape adjustments that were required to bring the various measurements into agreement. A weak, linking correlation with the ene:gy region below $6 \mathrm{MeV}$ was assumed. 
2. $\mathrm{MT}=103,107$

Variances were obtained by roughly constructing $\pm 2 \sigma$ curves Jounding the experimental data. Correlations with energy were based upon estimates of systematic errors in the measurements used for the evaluation. Near thresholds, progressively larger (fractional) uncertainties were assumed. Estimates of the covariances are probably poorest near the thresholds and where the cross sections are smallest, that is, where the variances are largest.

3. $\mathrm{MT}=2,4$

At energies below $13 \mathrm{MeV}$ for $\mathrm{MT}=4$ and above $13 \mathrm{MeV}$ for $\mathrm{MT}=2$, covariances were estimated as described in Sec. II.b.2. In the case of $M T=4$, uncertainties in the individual discreco inelastic reactions were combined to obtain the covariances.

The MT $=2$ cross section was derived below $13 \mathrm{MeV}$ by subtraction of the nonelastic from the total, and $\mathrm{MT}=4$ was derived above $13 \mathrm{MeV}$ from the total, elastic, and reaction cross sections. This information was used to derive the $\mathrm{MT}=2$ covariances below $13 \mathrm{MeV}$ and the $\mathrm{MT}=4$ covariances above $13 \mathrm{MeV}$. These data are given explicitly in MF $=33$, that is, the option to use NC-type subsections to represent derived quantities was not used.

\section{c. Correlations Across Reaction Types}

Derivation of the elastic cross section from the other reactions below $13 \mathrm{MeV}$ leads to correlations of the type $\mathrm{MT}=2 ; \mathrm{MT} 1=1,4,103,107$. Similarly, derivation of $\mathrm{MT}=4$ above $13 \mathrm{MeV}$ results in correlations of the type $\operatorname{MT}=4 ; \operatorname{MT} 1=1,2,103,107$. These correlations are also given explicitly in the file, that is, NC-type subsections are not used to represent the derived quantities. Explicit representation of the derived covariances required extensive use of $L B=3$ in the NI-type subsections. 
CROSS SECTION COVARIANCES FOR ${ }^{27}$ Al

by

D. G. Foster, Jr. and D. W. Muir

\section{Data Summary}

Covariance data are included in $M F=33$ for $M T=1,2,4,16,102,103$, and 107 of the ${ }^{27} \mathrm{~A} 1$ evaluation for the full energy range of $10^{-5} \mathrm{eV}$ to $20 \mathrm{MeV}$. In a1l instances, the covariances are developed from two basic components: (1) a short range or locally correlated component resulting from statistical or localized errors in measurements or knowledge of structure, and (2) longer range correlated components resulting from systematic errors in the measurements used, or from the analysis procedure itself.

Covariance data are not provided for $\mathrm{MT}=51-90,104$, and 105, although discrete cross sections are given for these reactions in $M F=3$. Similarly, there are no covariances for gamma-ray production data (MF $=12-15$ ) or for neutron angular and energy distributions ( $M F=4,5)$.

II. Methodology

a. $\mathrm{MT}=1$

Diagonal elements of the covariance matrix are taken from running fits to the cross section data (single- or double-peaked Breit-Wigner shapes in the structured region and polynomial fits in the smooth regions). Correlations in the errors reflect: (1) dependence on particular time-of-flight measurements over broad ranges of energy; (2) energy-dependent normalization of the Columbia measurements to join smoothly to other weasurements below $4.6 \mathrm{keV}$ and above 189 $\mathrm{keV}$; and, (3) correlations induced by the $1 / \mathrm{v}$ fit below $4.6 \mathrm{keV}$.

b. $M T=16,102,103,107$

Variances were estimated from dispersion of the experimental data and from quoted experimental errors. Correlations with energy were based upon estimates of systematic errors in the measurements used for the evaluation. Near thresholds, progressively larger (fractional) uncertainties were assumed. Es- 
timates of the covariances are probably poorest near the thresholds and where the cross sections are smallest, that is, where the variances are largest.

c. $\mathrm{MT}=2$

From $10^{-5} \mathrm{eV}$ to $9 \mathrm{MeV}$, the $\mathrm{MT}=2$ covariances are represented as derived from the other reaction types, using NC-type sub-subsections with LTY $=0$. Similarly, from 17 to $20 \mathrm{MeV}$ the covariances are derived from $M T=1$. In the energy range 9 to $17 \mathrm{MeV}$, the covariances were obtained as described in Sec. II,b.

d. $\mathrm{MT}=4$

From threshold to $9 \mathrm{MeV}_{\text {s }}$ the $\mathrm{MT}=4$ covariances were obtained as described in Sec. II.b. From 9 to $20 \mathrm{MeV}$, the covariances are represented as derived from the other reaction types, using NC-type sub-subsections with $\mathrm{LTY}=0$.

e. Correlations Across Reaction Types

The derivation of MT $=2$ from the other reactions below $9 \mathrm{MeV}$ leads to correlations of the type MT $=2$; MT1 $=1,4,102,103,107$. Similarly, the derivation of $M T=4$ above $9 \mathrm{MeV}$ results in correlations of the type $M T=4$; MT1 $=1,2,16,102,103,107$. Finally, correlations of the type MT $=1$; MT1 = 2 occur from 17 to $20 \mathrm{MeV}$. In all cases, the correlations are given implicitly through use of NC-type, LTY $=0$ sub-subsections. 